\title{
SELECTED WATER-LEVEL RECORDS \\ FOR WESTERN OKLAHOMA, 1975-1976
}

U. S. GEOLOGICAL SURVEY

0pen-File Report 77-239

Prepared in cooperation with the OKLAHOMA WATER RESOURCES BOARD

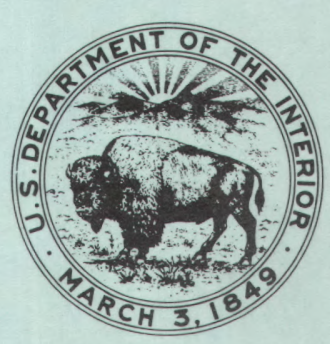




\section{UNITED STATES DEPARTMENT OF THE INTERIOR Geological Survey}

\section{SELECTED WATER-LEVEL RECORDS \\ FOR WESTERN OKLAHOMA, 1975-1976}

By Robert L. Goemaat

Open-File Report 77-239

Prepared in cooperation with the OKLAHOMA WATER RESOURCES BOARD

Oklahoma City, Oklahoma 



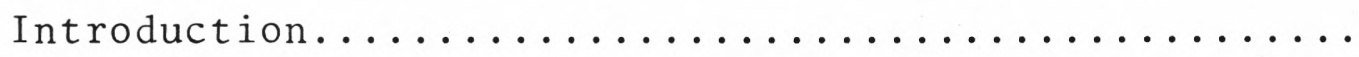

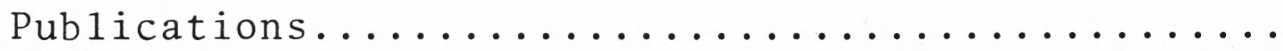

Metric-English equivalents............... 3

We11-numbering system................. 4

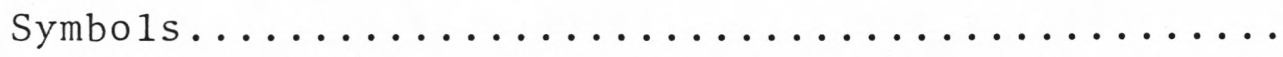

Counties for which water levels are published:

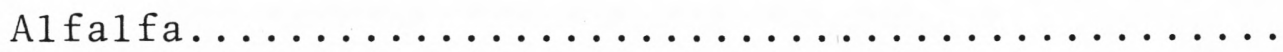

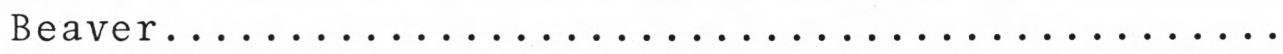

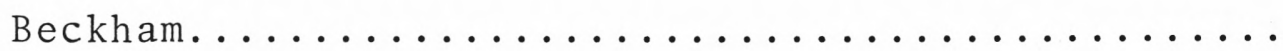

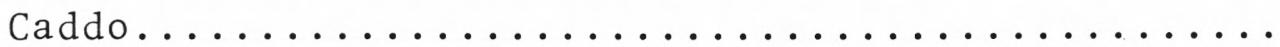

Cimarron.

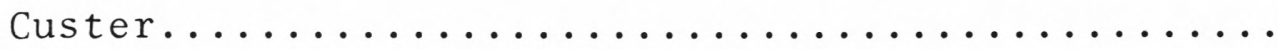

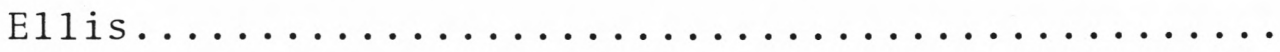

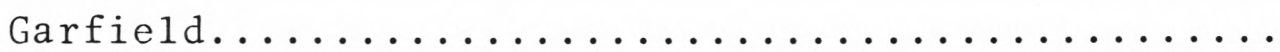

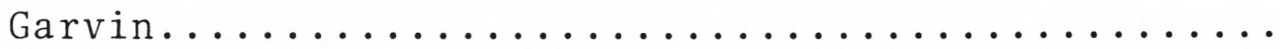

Grant

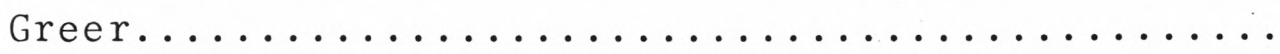

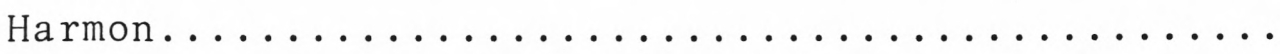

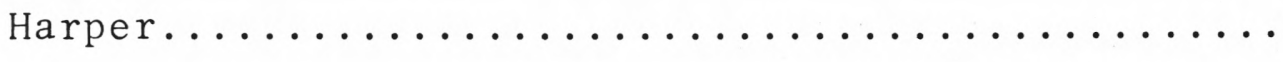

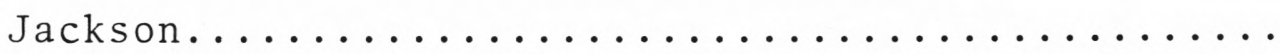

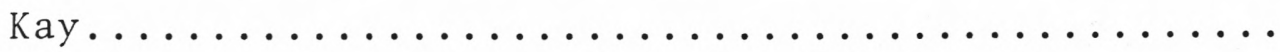

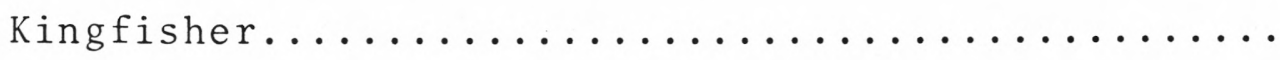

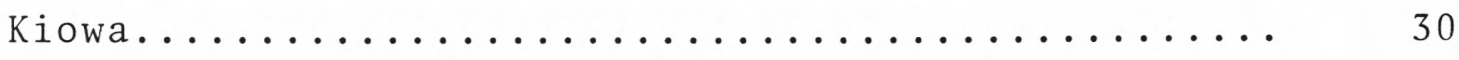

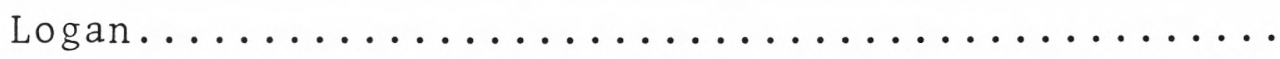


Major.............................. 31

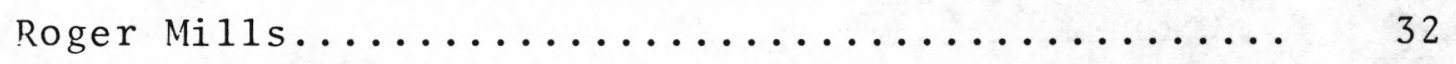

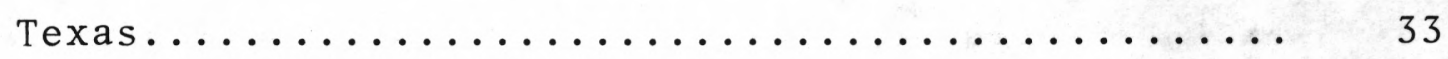

Tillman............................. 41

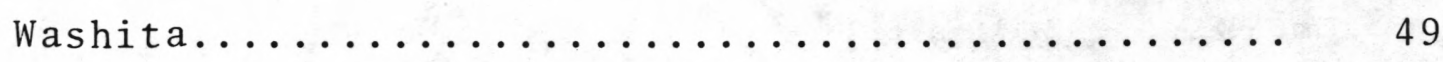

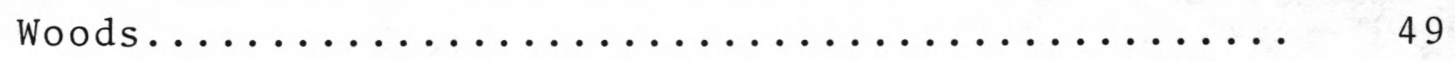

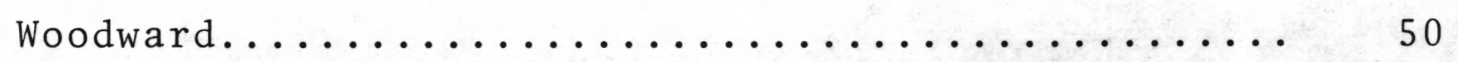

\section{LLUSTRATI ONS}

Figure 1.--Map showing counties with observation wells measured annual1y, 1975-76, and the number

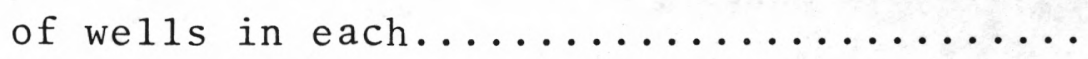

TABLES

Table 1.--Water levels and water-1evel changes........ 
SELECTED WATER-LEVEL RECORDS FOR WESTERN OKLAHOMA, 1975-1976

by Robert L. Goemaat

\section{INTRODUCTION}

A systematic program to collect water-level records in Oklahoma began in 1937. The objectives of this program are (1) to provide long-term records of water-level fluctuations in representative wells, (2) to facilitate the prediction of water-level trends and indicate future availability of groundwater supplies, and (3) to provide information for use in basic research.

Water-1evel data in table 1 are from wells that are measured annually, prior to the irrigation season to achieve the most natural representation of the static water level. Water-level measurements listed in the column under 1975 may have been made during December, 1974 or January or February, 1975. Measurements 1isted in the column 1976 may have been made during December, 1975 or January or February, 1976. Figure 1 shows the counties and number of wells therein, where data were obtained for this report.

The stratigraphic nomenclature and age determinations used ir this report are those accepted by the Oklahoma Geological Survey and do not necessarily agree with those of the U.S. Geological Survey.

Records of water levels in Oklahoma are collected through a cooperative program by the U.S. Geological Survey and the Oklahoma Water Resources Board. The records are tabulated and puolished by the U.S. Geological Survey on an annual basis. 


\section{Publications}

Publication of ground-water-leve1 data for the United

States in Water-Supply Papers was begun by the Geological Survey in 1935. From 1935 through 1939, a single Water-Supply Paper for each year covering the entire nation was issued (WaterSupply Papers $777,817,840,845$, and 886). From 1940 through 1974 , separate Water-Supply Papers were issued for 6 sections of the United States. Water-level data for Oklahoma are in the Water-Supply Papers listed below, each report containing one or more calendar years (January-December) of data.

\begin{tabular}{lccc}
\hline $\begin{array}{l}\text { Calendar } \\
\text { Year }\end{array}$ & Water-Supply Paper & $\begin{array}{c}\text { Calendar } \\
\text { Year }\end{array}$ & Water-Supply Paper \\
\hline 1935 & 777 & 1945 & 1099 \\
1936 & 817 & 1948 & 1129 \\
1937 & 840 & 1949 & 1159 \\
1938 & 845 & 1950 & 1168 \\
1939 & 886 & 1951 & 1194 \\
1940 & 090 & 1952 & 1244 \\
1941 & 939 & 1953 & 1268 \\
1942 & 947 & 1954 & 1324 \\
1943 & 989 & 1955 & 1407 \\
1944 & 1019 & $1956-59$ & 1549 \\
1945 & 1026 & $1960-64$ & 1824 \\
1946 & 1074 & $1965-69$ & 1979 \\
& & $1970-74$ & 2172 \\
\hline
\end{tabular}


In addition to the Water-Supply Papers listed above, the U.S. Geological Survey, in cooperation with the Oklahoma Water Resources Board, has published the following open-file reports on water levels in Oklahoma:

Ground-water levels in observation wells in Oklahoma, 1956-60

Ground-water levels in observation wells in 0k1ahoma, 1961-62

Ground-water levels in observation wells in 0klahoma, 1963-64

Ground-water levels in observation wells in 0klahoma, 1965-66

Ground-water levels in observation wells in Oklahoma, 1967-68

Ground-water levels in observation wells in Oklahoma, 1969-70

Ground-water levels in observation wells in Oklahoma, 1971-74

Records of water-1eve1 measurements in wells in the Oklahoma Panhandle, 1966-70

Records of water-level measurements in wells in the Oklahoma Panhandle, 1971-72

Selected water-1evel records for western Oklahoma, 1950-1975

\section{Metric-English Equivalents}

English units used in the report may be converted to metric units by the following conversion factors:

Multiply English Units by $\underline{\text { To obtain SI Units }}$

acres (acres)

$0.004047 \mathrm{~km}^{2}$ (square kilometer)

ft $($ feet)
.3048

2.590

m (meter)

$\mathrm{mi}^{2}$ (square mile) $\mathrm{km}^{2}$ (square kilometer) 
The standard method of giving location by fractional section, section, township, and range is replaced by the method illustrated in the diagram below. The location of the well indicated by the dot normally would be described as NE $1 / 4$ SW $1 / 4 \mathrm{NW} 1 / 4$ sec. 6, T. 24 N., R. 13 E. The method used in this report reverses the order and indicated quarter subdivisions of the section by letters. By this method the location of the wel1 is given as $24 \mathrm{~N}-13 \mathrm{E}-06 \mathrm{BCA}$. The final digit (1) is the sequential number of a well within the smallest fractional subdivision. For some wells the location is known only to the quarter section (160-acre tract) or quarter-quarter section (40-acre tract) and is so indicated by the we11 number.

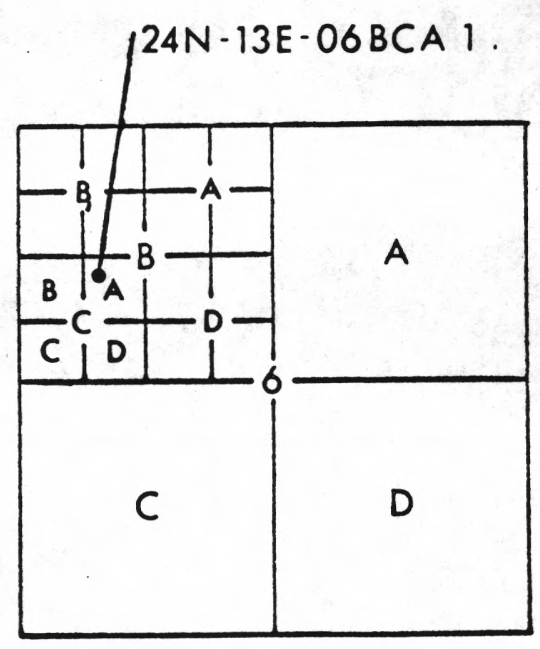




\section{$\underline{\text { SYMBOLS }}$}

Symbols are used in table 1 to indicate the following:

$\underline{A}$, well being pumped when visited for measurement; $\underline{B}$, well pumped recently, prior to visit; $\underline{\mathrm{C}}$, nearby well pumping when observation well was measured; E, estimated depth to water; $\underline{F}$, observation well dry when measured; $\underline{\mathrm{H}}$, tape measurement included with water-levels obtained by automatic recorder: $\underline{K}$, unable to get measuring device to the water-1evel; $\underline{L}$, observation well has been destroyed; $\underline{M}$, measurements of observation well have been discontinued; $\underline{N}$, observation well not visited; EOM, end of month; LSD, 1and-surface datum. 


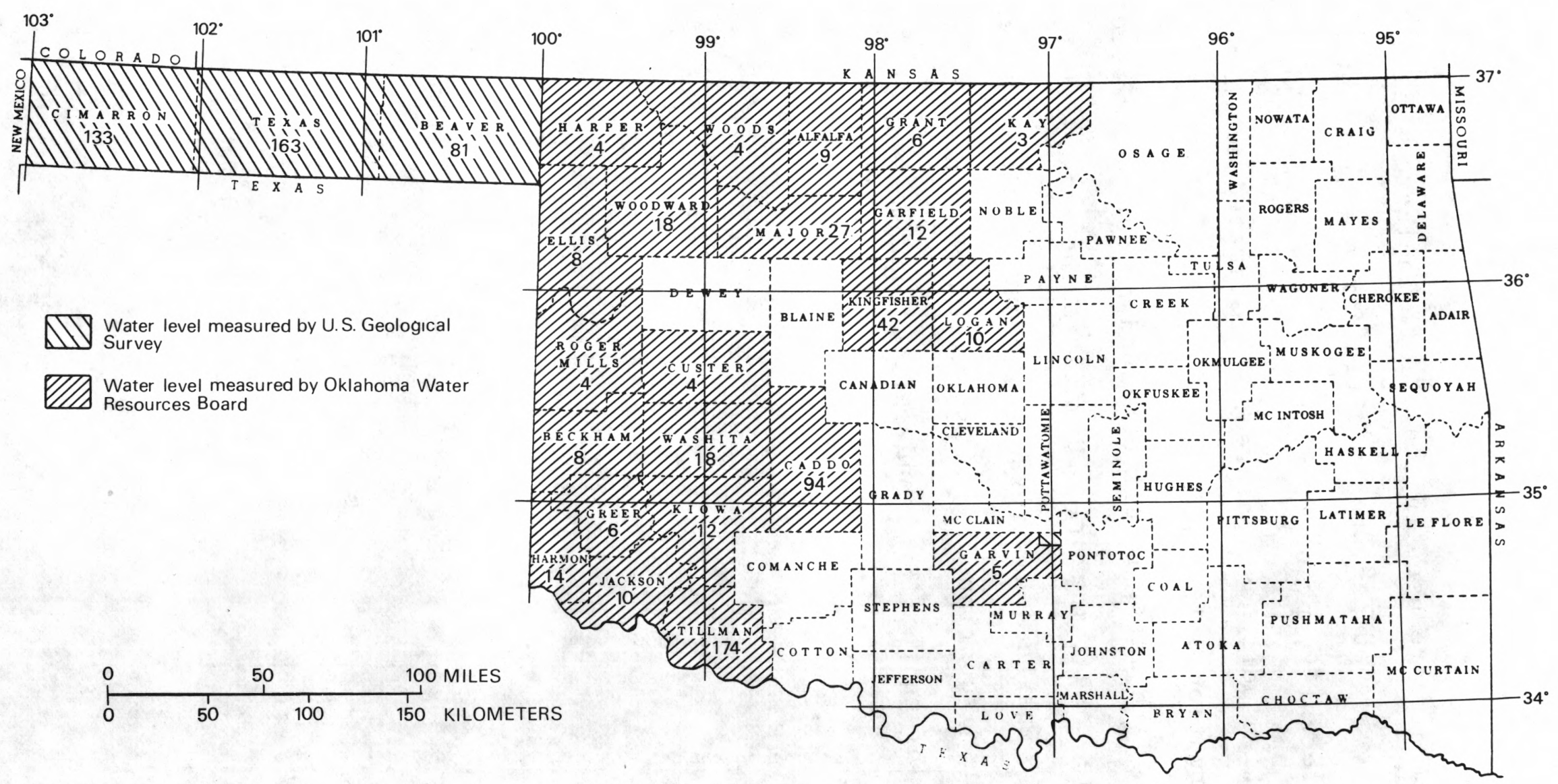

Figure 1.--Map showing counties (hatched) with observation wells measured annually, 1975-76, and the number of wells in each. 
Table 1.-Water levels and water-level changes.

alFalFa COUNTY

\begin{tabular}{|c|c|c|c|c|c|c|c|c|c|}
\hline \multirow{2}{*}{\multicolumn{2}{|c|}{$\begin{array}{c}\text { WELL } \\
\text { LOCATIUN }\end{array}$}} & \multirow{2}{*}{\multicolumn{3}{|c|}{$\begin{array}{l}\text { PRINCIFAL } \\
\text { AGUIFER }\end{array}$}} & \multicolumn{2}{|c|}{$\begin{array}{l}\text { NATER LEVEL } \\
1975\end{array}$} & \multirow{2}{*}{$\begin{array}{l}\text { NATER } \\
\text { LEVEL } \\
\text { EHANGE } \\
1975-76\end{array}$} & \multicolumn{2}{|c|}{$\begin{array}{l}\text { WATEKK LEVEL } \\
1976\end{array}$} \\
\hline & & & & & DATE & DEPTH & & DATE & DEPTH \\
\hline $025 N=011 A-16$ & $D O C$ & 1 & TERRACE & DEPUSITS & $01-23-75$ & 7.01 & 0.77 & $01-16-70$ & 0.24 \\
\hline $027 N=009 w=09$ & $\forall \forall A$ & 1 & TERIACE & DEPOSITS & $01-24-75$ & 3.67 & 0.52 & $01-16-76$ & 3.15 \\
\hline $027 N-011 N=09$ & OCC & 1 & TERRACE & DEPUSITS & $01-24-75$ & 10.25 & -1.20 & $01-15-76$ & 11.45 \\
\hline $027 N-011 w-14$ & vot & 1 & TEWHACE & DEPUSITS & $01-24-75$ & 5.00 & -2.64 & $01-15-76$ & 7.64 \\
\hline $27 N-011 N-23$ & 480 & 1 & TERRACE & DEPOSITS & $01-24-75$ & 9.72 & 0.09 & $01-15-70$ & 9.03 \\
\hline $028 N-010 W=20$ & AUO & 1 & TERRACE & DEPOSITS & & 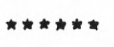 & 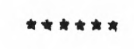 & $01-16-76$ & $\star \star \star \star \star \star \star \star L L$ \\
\hline $028 N=111 N-10$ & Doo & 1 & TERKACE & DEPOSITS & $01-24-75$ & 12.80 & 1.50 & $01-15=70$ & 11.30 \\
\hline $02 \forall N-C 11 w-27$ & OAU & 1 & TERRACE & DEPOSITS & $01-24-75$ & 0.23 & 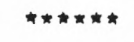 & $01-16-70$ & $\star \star \star \star \star \star A$ \\
\hline $29 N=009 n-18$ & CUC & 1 & TERRACE & DEPOSITS & $01-24-75$ & 0.49 & -1.03 & $01-10-70$ & 7.52 \\
\hline
\end{tabular}


Table 1.-Water levels and water-level changes--Continued.

BEAVER COUNTY

\begin{tabular}{|c|c|c|c|c|c|c|c|c|c|}
\hline NELL & & & PRIN & VCIPAL & $\begin{array}{r}\text { WATER } \\
197\end{array}$ & EYEL & $\begin{array}{l}\text { WATER } \\
\text { LEVEL }\end{array}$ & $\begin{aligned} \text { NATER } \\
197\end{aligned}$ & DEVEL \\
\hline LOCATION & & & $A Q U$ & JIFER & DATE & DEPTH & $\begin{array}{r}\text { CHANGE } \\
1975-76\end{array}$ & UATE & DEPTH \\
\hline $001 N-020 E-16$ & COD & 1 & UGALLALA & FURMATIUN & $01-21-75$ & 102.01 & -0.49 & $01-13-76$ & 102.50 \\
\hline $001 N-021 E-05$ & UDU & 1 & OGALLALA & FURMATION & $01-27-75$ & 189.39 & 0.01 & $01-13-76$ & 189.38 \\
\hline $001 N-021 E-32$ & $\triangle B C$ & 1 & OGALLALA & FURMAIIUN & $01-21-75$ & 243.04 & 0.71 & $01-13-76$ & 242.33 \\
\hline $001 N=022 E-12$ & $\Delta C_{0}$ & 1 & OGALLALA & FOKMATION & $01-21=75$ & 189.80 & -2.48 & $01-13-76$ & 192.28 \\
\hline $001 N-022 E-33$ & 000 & 1 & DGALLALA & FCRMATIUN & $01-21-75$ & 238.11 & -2.09 & $01-13-76$ & 240.20 \\
\hline $001 N-022 E-34$ & $\operatorname{coc}$ & 1 & OGALLALA & FORMATION & $01-21-75$ & 243.43 & -2.44 & $01-13-76$ & 245.87 \\
\hline $001 N-023 E-08$ & $D C \dot{\theta}$ & 1 & DGALLALA & FURMATIUN & $01-24-75$ & 210.01 & -1.40 & $01-14-76$ & 212.01 \\
\hline $001 N-023 E-19$ & UAO & 1 & OGALLALA & FORMATION & $01-21-75$ & 233.82 & -1.59 & $01-13-76$ & 235.41 \\
\hline $001 N-023 E-23$ & $\Delta \Delta y$ & 1 & UIGALLALA & FORMATION & $01-21-75$ & 160.03 & -0.21 & $01-13-70$ & 160.24 \\
\hline $001 N-023 t-26$ & $B 80$ & 1 & OGALLALA & FORMATION & $01-21-75$ & 222.35 & -3.18 & $01-13=76$ & 225.53 \\
\hline $001 N-023 E-29$ & $B \Delta C$ & 1 & OGALLALA & FORMATIUN & $01-21-75$ & 239.29 & -4.69 & $01-13-70$ & 243.98 \\
\hline $001 N-024 t-01$ & $A \in D^{\circ}$ & 1 & OGALLALA & FURMATIUN & $01-21-75$ & 221.09 & $=0.41$ & $01-14-76$ & 221.50 \\
\hline $001 N=025 E=07$ & CAA & 1 & OGALLALA & FURMATIUN & $01-21-75$ & 62.57 & 0.17 & $01-14=76$ & 62.40 \\
\hline $001 N-020 t-05$ & vOC & 1 & DGALLALA & FORMATION & $01-20-75$ & 85.89 & -0.21 & $01-12-76$ & 86.10 \\
\hline $001 N=020 t-27$ & $\cup C D$ & 1 & ALLUVIUM & ${ }^{\circ}$ & $01-20-75$ & $\star \star \star \star \star \star \star \star k K$ & $* * * *$ & $01-12-76$ & 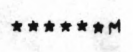 \\
\hline $001 N-020 E=30$ & $\Delta \Delta O$ & 1 & ALLUVIUIM & & $01-20-75$ & $\star * * * * * k$ & $\star \star \star \star \star \star \star \star *$ & $01-12-76$ & $\star * \star * * * * \pi$ \\
\hline $001 N=026 E-30$ & $\triangle 0 C$ & 1 & OGALLALA & FURMATION & $01-20-75$ & 169.09 & -0.31 & $01-14=76$ & 170.00 \\
\hline $001 N-027 E=00$ & $A C_{O}$ & 1 & ALLUVIUM & & $01-24=75$ & 19.45 & 0.15 & $01-14-70$ & 19.30 \\
\hline $001 N-027 t-21$ & $\Delta \Delta$ & 1 & UGALLALA & FORMATION & $01-20-75$ & 39.41 & -0.50 & $01-12-76$ & 39.91 \\
\hline $001 N=027 E-35$ & OAC & 1 & UGALLALA & FUAMATIUN & $01-20-75$ & 141.02 & -0.15 & $01-12-76$ & 141.77 \\
\hline $001 N=028 E-02$ & $O C A$ & 1 & IJGALLALA & FURMATIUN & $01-20-75$ & 152.07 & -0.16 & $01-12-76$ & 152.23 \\
\hline $001 N=028 E=34$ & $O A A$ & 1 & OGALLALA & FURMATIUN & $01-20-75$ & $\star \star \star * * * \hbar k$ & $\star \star \star \star \star \star \star$ & $01-12-76$ & $* *$ \\
\hline $002 N-020 E-U 1$ & $\triangle A A$ & 1 & JGALLALA & FOPMATION & $01-21-75$ & 51.39 & -0.21 & $01-13-76$ & 51.00 \\
\hline
\end{tabular}


Table 1.-Water levels and water-level changes--Continued.

$$
\text { JEAVER COUNTY }
$$

\begin{tabular}{|c|c|c|c|c|c|c|c|c|c|}
\hline WELL & & & PWIR & NCIPAL & $\begin{array}{r}\text { WATER } \\
197\end{array}$ & $5 E V E L$ & $\begin{array}{l}\text { NATER } \\
\text { LEVEL }\end{array}$ & $\begin{array}{r}\text { WATER } \\
19\end{array}$ & LEVEL \\
\hline LDCATIUA & & & 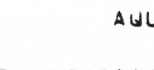 & UIFER & DATE & DEPTH & $\begin{array}{l}\text { CHANGE } \\
1975-76\end{array}$ & DATE & DEFTH \\
\hline $002 N-021 E-11$ & cuv & 1 & OGALLALA & FORMATION & $01-21-75$ & 174.35 & -1.23 & $01-13-76$ & 175.58 \\
\hline $002 N-022 E-14$ & DCC & 1 & OGALLALA & FORMATIUN & $01-21-75$ & 100.50 & 5.36 & $01-13-76$ & 95.14 \\
\hline $002 N=022 E-27$ & OAD & 1 & UGALLALA & FORMATION & $01-21-75$ & 124.83 & -0.93 & $01-13-70$ & 125.76 \\
\hline $002 N-023 E-13$ & $\triangle C C$ & 1 & UGALLALA & FORMATIUN & $01-21-75$ & 12.62 & -0.03 & $01-13-76$ & 12.65 \\
\hline $002 N-024 E-07$ & $C C O$ & 1 & UGALLALA & FORMATION & $01-21-75$ & 70.29 & -1.71 & $01-14-76$ & 78.00 \\
\hline $002 N-024 t-24$ & $C 8 \theta$ & 1 & OGALLALA & FURMATIUN & $01-21-75$ & 180.04 & -5.29 & $01-14-70$ & 191.33 \\
\hline $002 N=025 E-02$ & $B A U$ & 4 & OGALLALA & FORMATION & & 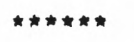 & $\star \star \star \star \star \star \star$ & & $\| \star \star \star \star \star \star *$ \\
\hline $002 V-025 E-17$ & $\triangle D D$ & 2 & UGALLALA & FORMATION & $01-21-75$ & 84.51 & -0.59 & $01-14-76$ & 05.10 \\
\hline $002 N=025 E-33$ & $A C A$ & 1 & UGALLALA & FORMATION & $01-24-75$ & 210.40 & -1.58 & $01-16-76$ & 211.98 \\
\hline $002 N=026 E=U 2$ & $8 B A$ & 1 & OGALLALA & FUKMATIUN & $01-20-75$ & 56.23 & -0.27 & $01-14-76$ & 56.50 \\
\hline $002 N=027 E-02$ & $A A C$ & 2 & ALLUVIUM & & $01-20-75$ & 18.99 & -0.41 & $01-14-70$ & 19.40 \\
\hline $002 N=027 E-15$ & $\theta 60$ & 1 & ALLLIVIUM & & $01-20-75$ & 10.03 & -0.22 & $01-14-76$ & 10.25 \\
\hline $002 N=027 E-21$ & $\cos$ & 2 & ALLUVIUM & & $01-20-75$ & 8.32 & -0.08 & $01-12-76$ & 8.40 \\
\hline $002 N=027 E-29$ & $U C D$ & 3 & ALLUVIUM & & $01-20-75$ & 10.32 & -0.08 & $01-12-76$ & 10.40 \\
\hline $002 N-027 E-31$ & ODA & 1 & ALLUVIUM & & $01-20-75$ & 9.26 & -0.94 & $01-12-70$ & 10.20 \\
\hline $003 N=024 E-18$ & BAA & 1 & UGALLALA & FURMATICN & $01-21-75$ & 110.736 & -0.378 & $01-13-76$ & 119.10 \\
\hline $003 N-020 E=06$ & $\operatorname{cec}$ & 1 & OGALLALA & FORMATION & $01-2 v-75$ & 82.43 & -2.16 & $01-14-76$ & 84.59 \\
\hline $003 N=026 t-10$ & $A A B$ & 1 & OGALLALA & FUKMATIUN & $01=20-75$ & 13.25 & -0.14 & $01-13-76$ & 13.39 \\
\hline $003 N-027 E-04$ & oCu & 1 & UGALLALA & FORMATION & & 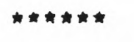 & 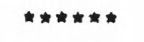 & $01-13-76$ & $\# \# \# \# \# * 1$ \\
\hline $003 N=027 E-14$ & $\mathrm{CC} \forall$ & 1 & UGALLALA & FOKMATION & $01-20-75$ & 80.55 & -0.17 & $01-13-76$ & 86.72 \\
\hline $004 N-020 E=04$ & $c \subset C$ & 1 & OGALLALA & FORMATIUN & & 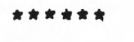 & 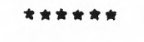 & $01-13-76$ & 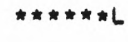 \\
\hline $004 N=020 E-19$ & $\mathrm{cos}$ & 1 & OGALLALA & FORMATION & $01-22-75$ & 98.79 & -2.20 & $01-13-76$ & 100.99 \\
\hline $004 N-020 E-22$ & CAD & 1 & OGALLALA & FURMATIUN & $01-22-75$ & 116.04 & -0.90 & $01-13-76$ & 110.94 \\
\hline
\end{tabular}


Table 1.--Water levels and water-level changes--Continued.

GEAVER COUNTY

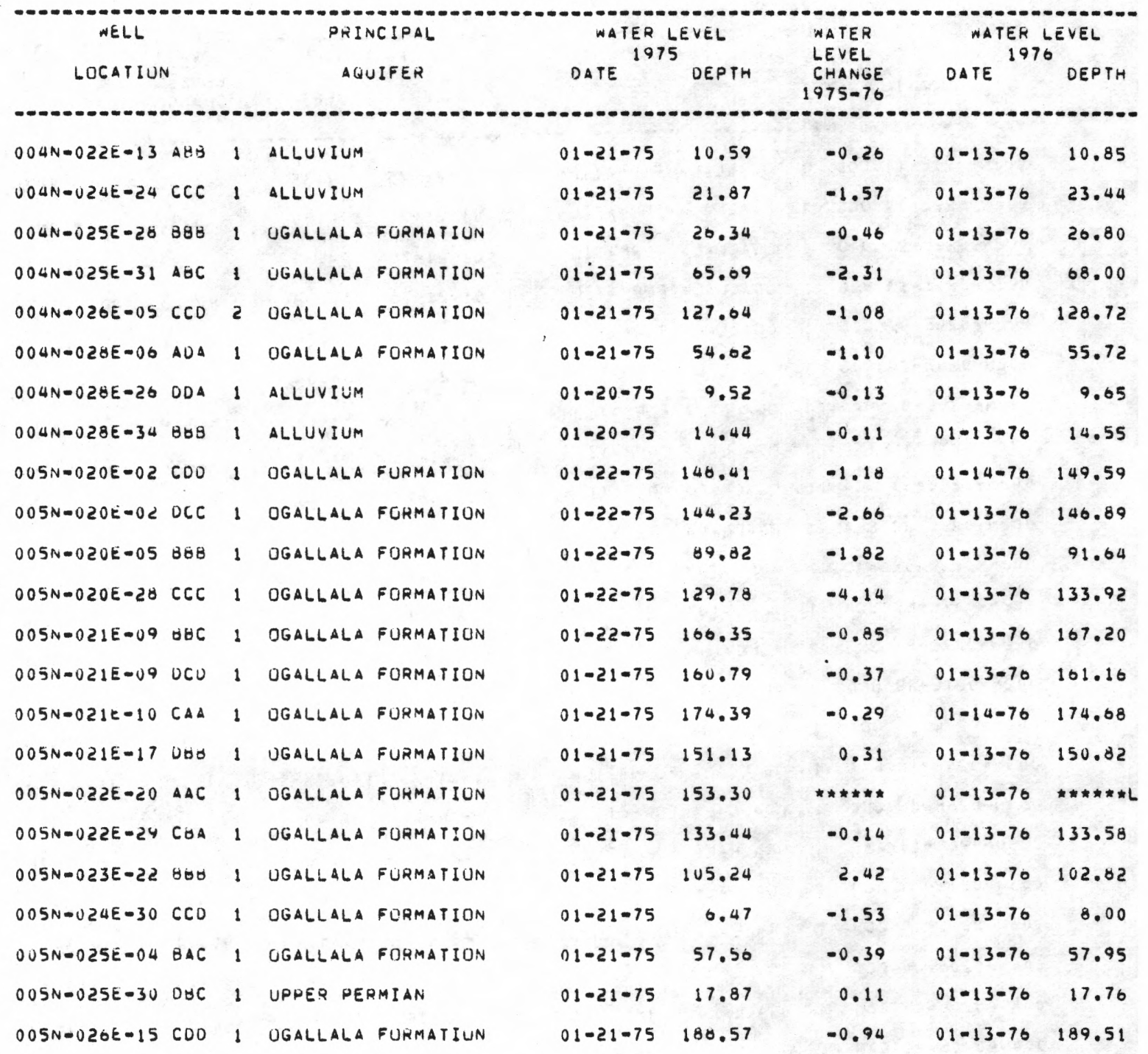


Table 1.--Water levels and water-level changes--Continued.

OEAVER CUUNTY

\begin{tabular}{|c|c|c|c|c|c|c|c|c|c|}
\hline \multirow{2}{*}{\multicolumn{2}{|c|}{$\begin{array}{c}\text { WELL } \\
\text { LOCATIUN }\end{array}$}} & \multirow{2}{*}{\multicolumn{3}{|c|}{$\begin{array}{l}\text { PRINCIPAL } \\
\text { AUUIFER }\end{array}$}} & \multicolumn{2}{|c|}{$\begin{array}{l}\text { WATER LEVEL } \\
1975\end{array}$} & \multirow{2}{*}{$\begin{array}{l}\text { HATER } \\
\text { LEVEL } \\
\text { CHANGE } \\
1975-76\end{array}$} & \multicolumn{2}{|c|}{$\begin{array}{l}\text { WATER LEVEL } \\
1976\end{array}$} \\
\hline & & & & & DATE & OEPTH & & DATE & OEPTH \\
\hline $0 O S N=027 E=09$ & $\triangle D D$ & 1 & OGALLALA & FORMATION & $01-21-75$ & 59.77 & 0.99 & $01-13-70$ & 58.78 \\
\hline $005 N-027 E-19$ & $d C B$ & 1 & DGALLALA & FUKMATION & $0 t-21-75$ & 159.89 & 3.05 & $01-13-70$ & 156.84 \\
\hline $005 N-027 E-35$ & CUC & 1 & DGALLALA & FURMATION & $01-21-75$ & 00.21 & 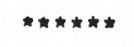 & $01-13-76$ & \#* \\
\hline $005 N=028 E=00$ & $A A A$ & 1 & OGALLALA & FURMATIUN & $01-21-75$ & 48.69 & -0.05 & $01-13-76$ & 49.34 \\
\hline $005 N-028 E-22$ & $\triangle B A$ & 1 & OGALLALA & FURMATION & $01-21-75$ & 20.19 & -0.07 & $01-12-70$ & 20.26 \\
\hline $005 N-028 E-27$ & $B C D$ & 1 & OGALLALA & FURMATION & $01-21-75$ & 57.58 & 0.25 & $0:-12-76$ & 57.33 \\
\hline $006 N=020 E-17$ & cou & 1 & DGALLALA & FORMATIUN & $01-22-75$ & 105 & 0.39 & $01-13-76$ & 104.68 \\
\hline $006 N=02 U E-35$ & $D C C$ & 1 & OGALLALA & FORMATIUN & $01-22-75$ & 140.40 & -4.61 & $01-13-76$ & 151.01 \\
\hline $000 N-021 E-18$ & $\operatorname{ccc}$ & 1 & OGALLALA & FORMATIUN & $01-21-75$ & 154.22 & -1.00 & $01-13-70$ & 155.22 \\
\hline $006 N-022 E-24$ & vou & 1 & DGALLALA & FDRMATIUN & $01-21-75$ & 102.10 & 0.90 & $01-13-70$ & 101.20 \\
\hline $006 N=022 E-32$ & AAL & 1 & OLALLALA & FORMATIUN & $01-21-75$ & 166.42 & 0.69 & $01-13-76$ & 165.73 \\
\hline $06 N=024 E=27$ & $\operatorname{ccc}$ & 1 & OGALLALA & FORMATION & & $\star \star \star \star \star \star$ & 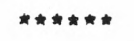 & $01-13-70$ & 110.07 \\
\hline
\end{tabular}

BECKHAM COUNTY

\begin{tabular}{|c|c|c|c|c|c|c|c|c|}
\hline \multirow{2}{*}{\multicolumn{2}{|c|}{ LOCATIUN }} & \multirow{2}{*}{\multicolumn{2}{|c|}{$\begin{array}{c}\text { PRINCIPAL } \\
\text { AUUIFER }\end{array}$}} & \multicolumn{2}{|c|}{$\begin{array}{l}\text { WATER LEVEL } \\
1975\end{array}$} & \multirow{2}{*}{$\begin{array}{l}\text { WATER } \\
\text { LEVEL } \\
\text { CHANGE } \\
\text { I975-70 }\end{array}$} & \multicolumn{2}{|c|}{$\begin{array}{l}\text { WATER LEVEL } \\
1976\end{array}$} \\
\hline & & & & DATE & DEPTH & & DATE & DEPTH \\
\hline $008 N-024 N=02$ & טUO & 1 & TERRACE DEPUSITS & $01-08-75$ & 25.03 & 1.36 & $02-11-76$ & 23.67 \\
\hline $008 N=024 N=09$ & AAO & 1 & TERRACE DEPOSITS & $01-08=75$ & 58.99 & 5.17 & $02-11-76$ & 53.82 \\
\hline $008 N-025 N-10$ & $\forall \Delta A$ & 1 & TERRACE UEPUSITS & $01-08-75$ & 44.02 & 3.05 & $02-11-76$ & 40.77 \\
\hline $009 N=025 k-19$ & $C C C$ & 1 & TERHACE DEPUSITS & $01-08-75$ & 18.12 & 0.42 & $02-11-70$ & 17.70 \\
\hline $009 N=025 N-31$ & 860 & 1 & TERRACE DEPLSITS & $01-08-75$ & $\star \star \star \star \star \star \star \star \star A$ & 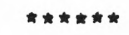 & $02-11-76$ & 10.84 \\
\hline $009 N=025 W=35$ & 000 & $!$ & TERKACE DEPUSITS & $01-08-75$ & 52.81 & 9.52 & $02-11-70$ & 43.29 \\
\hline $009 N=026 N=08$ & $8 C 0$ & 1 & TERRACE DEFOSITS & $01-08-75$ & 50.99 & -2.03 & $02-11-70$ & 59.02 \\
\hline $009 N=026 N=33$ & 000 & 1 & TERRACE DEPDSITS & $01-08-75$ & 8.05 & -0.75 & $02-11-70$ & 9.40 \\
\hline
\end{tabular}


Table 1.--Water levels and water-level changes--Continued.

CADOO COUNTY

\begin{tabular}{|c|c|c|c|c|c|c|c|c|c|c|}
\hline \multirow{2}{*}{ LOCATIUN } & & \multirow{2}{*}{ 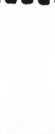 } & \multicolumn{3}{|c|}{ PRINCIPAL } & \multicolumn{2}{|c|}{$\begin{array}{l}\text { WATER LEVEL } \\
1975\end{array}$} & \multirow{2}{*}{$\begin{array}{l}\text { WATER } \\
\text { LEVEL } \\
\text { CHANGE } \\
1975-76\end{array}$} & \multicolumn{2}{|c|}{$\begin{array}{l}\text { WATER LEVEL } \\
1970\end{array}$} \\
\hline & & & & AQUIFER & & DATE & DEPTH & & UATE & DEPTH \\
\hline $8 N=011 W=07$ & $A A D$ & 1 & RUSH & SPFINGS & SANUSTONE & $12-16-74$ & 82.94 & 7.74 & $12-02-75$ & 75.20 \\
\hline $08 N=011 N=08$ & CAD & 1 & RUSH & SPRINGS & SANOSTONE & $12-16-74$ & $\star \star \star \star \star \star \star \star K$ & $\# \star \star \star$ & $12=02-75$ & $\star \star \star \star \star \star \star \star \star * k$ \\
\hline $08 N=011 W-18$ & cus & 1 & RUSH & SPRINGS & SANDSTONE & $12-16-74$ & 78.47 & 2.17 & $12-02-75$ & 70.30 \\
\hline $08 N-011 W-19$ & CAC & 1 & RUSH & SPRINGS & SANUSTUNE & $12-16-74$ & 80.73 & 3.23 & $12-02-75$ & 77.50 \\
\hline $08 N-012 W=01$ & $B A C$ & 1 & RUSH & SPKINGS & SANDSTONE & & 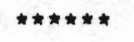 & 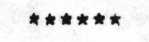 & $12-02-75$ & 53.30 \\
\hline $0 O N=012 N=06$ & $A C B$ & 1 & RUSH & SPRINGS & SANUSTONE & $12-17-74$ & 35.49 & 2.99 & $12-03-75$ & 32.50 \\
\hline $08 N=012 m=07$ & $A C A$ & 1 & RUSH & SPRINGS & SANUSTONE & $12-17-74$ & 43.53 & 1.63 & $12-02-75$ & 41.90 \\
\hline $08 N-012 N-11$ & $D C B$ & 1 & RUSH & SPRINGS & SANDSTUNE & $12-16-74$ & 03.05 & 4.65 & $12-02-75$ & 58.40 \\
\hline $08 N-012 n-12$ & DAB & 1 & RUSM & SPRINGS & SANUSTONE & $12-16-74$ & 74.38 & 2.68 & $12-02-75$ & 70 \\
\hline $08 N-012 w-27$ & $A A U$ & 1 & RUSH & SPRINGS & SANUSTONE & $12-16-74$ & $\star * * * * \hbar x$ & 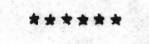 & $12-02-75$ & $\approx \# M$ \\
\hline $08 N=012 w-28$ & 883 & 1 & RUSH & SPRINGS & SANOSTONE & & $\star \star$ & $* * *$ & $12-02-75$ & 62.40 \\
\hline $08 N-012 w-34$ & $\operatorname{cco}$ & 1 & RUSH & SPRINGS & SANUSTUNE & $12-16-74$ & 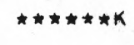 & 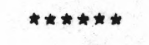 & $12-02-75$ & 90 \\
\hline $108 N-013 w-01$ & $\triangle B C$ & 1 & RUSH & SPRINGS & SANOSTONE & & 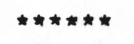 & 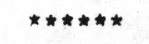 & & $\star \star *$ \\
\hline $08 N-013 n=03$ & $\cos$ & 1 & RUSH & SPRINGS & SANDSTONE & $12-17-74$ & 37 & -1.24 & $12-03-75$ & 38.30 \\
\hline $006 N-013 w-20$ & ANA & 1 & RUSH & SPRINGS & SANUSTUNE & $12-17-74$ & 69.37 & 0.37 & $12-03-75$ & 00 \\
\hline $008 N-013 w=25$ & $\triangle C U$ & 1 & RUSH & SPRINGS & SANUSTUNE & $12-07-74$ & 66.52 & 1.42 & $12-02-75$ & 65.10 \\
\hline $09 N=011 N=07$ & $C A C$ & 1 & RUSH & SPRINGS & SANUSTUNE & & $\star *$ & * & $12-03-75$ & 00 \\
\hline $009 N=0111 n=08$ & $A A U$ & 1 & RUSh & SPRINGS & SANDSTUNE & $12-18-74$ & 47.64 & 0.64 & $12-03-75$ & 47.00 \\
\hline $009 N-011 w-10$ & CCC & 1 & RUSH & SPRINGS & SANUSTONE & $12-17-74$ & 77.42 & 1.70 & $12-03-75$ & 75.72 \\
\hline $09 N-011 N-17$ & $\triangle 00$ & 1 & RUSH & SPRINGS & SANOSTUNE & $12-18-74$ & 10 & $\star \star \star \star * \star * \star$ & & 2 \\
\hline $009 N=011 m-30$ & $A A C$ & 1 & RUSH & SPRINGS & SANOSTONE & $12-17-74$ & 76.55 & 2.55 & $12=03=75$ & 74.00 \\
\hline $09 N-012 m=01$ & $U C \theta$ & 1 & RUSH & SPRINGS & SANOSTONE & $12-17-74$ & 91.29 & 1.17 & $12-03-75$ & 90.12 \\
\hline $09 N=012 W=00$ & DOd & 2 & RUSH & SPKINGS & SANDSTUNE & $12-16-74$ & 60.90 & 4.63 & $12=03-75$ & 56.27 \\
\hline
\end{tabular}


Table 1.--Water levels and water-level changes--Continued.

CAOUO COUNTY

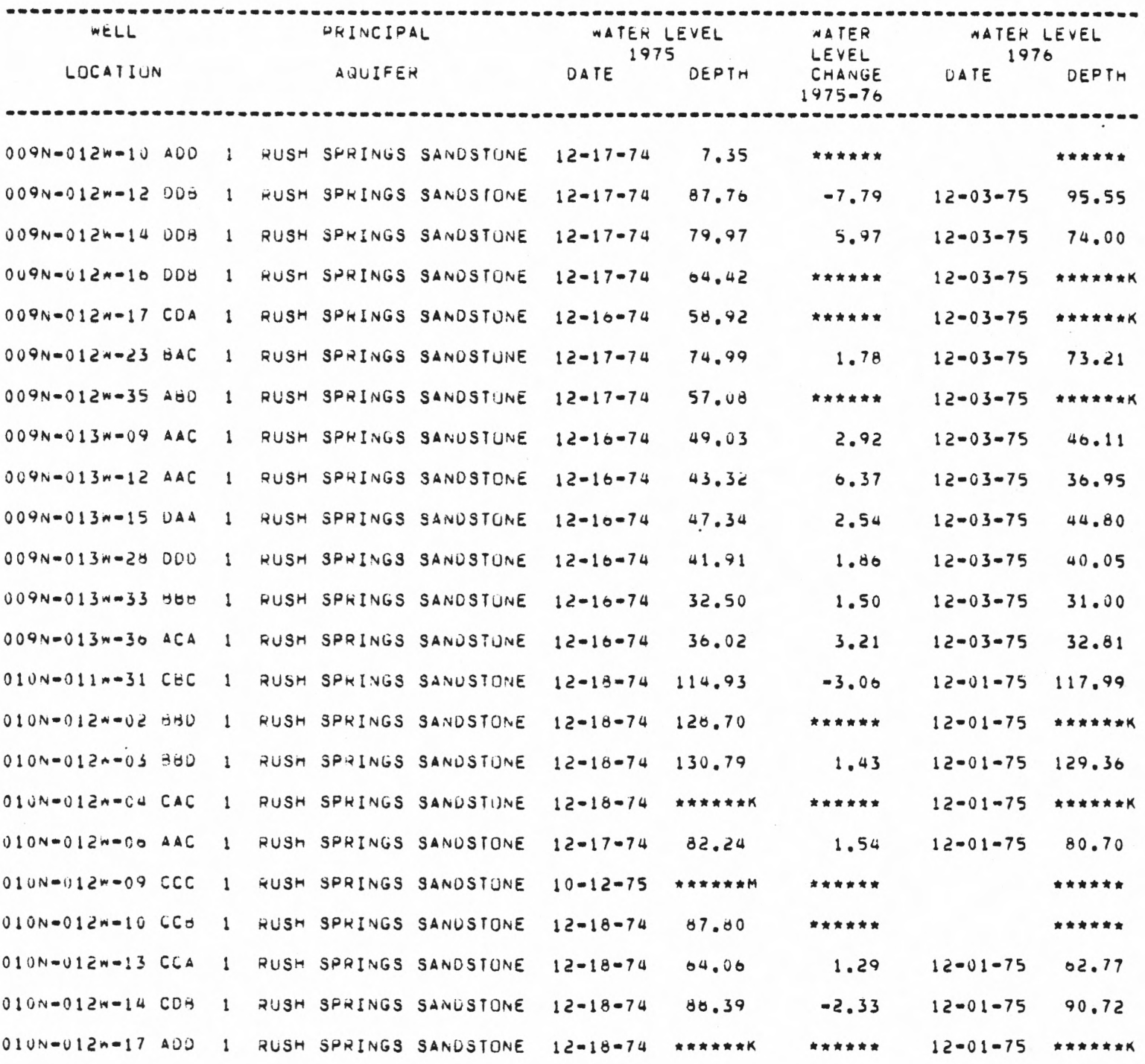


Table 1.--Water levels and water-level changes--Continued.

CAUDU COUNTY

\begin{tabular}{|c|c|c|c|c|c|c|c|c|c|c|}
\hline NELL & & & & RRINCIPA & & $\begin{array}{r}\text { WATER } \\
197\end{array}$ & EVEL & $\begin{array}{l}\text { MATER } \\
\text { LEVEL }\end{array}$ & $\begin{array}{r}\text { WATER } \\
197\end{array}$ & EVEL \\
\hline LUCAYIUN & & & & $A Q U I F E R$ & & DATE & DEPTH & CHANGE & DATE & DEPTH \\
\hline $1 U N=012 m-2 U$ & OAC & 1 & RUSH & SPRINGS & SANUSIONE & $12-18-74$ & $82: 42$ & 2.84 & $12=01-75$ & 79.58 \\
\hline $010 N=012 W=31$ & 000 & 1 & RUSH & SPRINGS & SANUSTUNE & $12-18-74$ & 73.67 & 0.12 & $12-01-75$ & 73.55 \\
\hline $10 N=012 n-33$ & $A \in C$ & 1 & RUSH & SPRINGS & SANOSTONE & $12-18-74$ & $\star \star \star \star \star \star \star \star \star k$ & $\star \star \star \star \star \star \star \star \star$ & $12=01-75$ & 92.26 \\
\hline $10 N-012 m=34$ & U⿺辶 & 1 & RUSH & SPRINGS & SANDSTUNE & $12-18-74$ & 65.36 & 7.09 & $12-01-75$ & 58.27 \\
\hline $10 N=012 n=35$ & $C \forall A$ & 1 & RUSH & SPRINGS & SANDSTONE & $12-18-74$ & 37.16 & 2.98 & $12-03-75$ & 34.18 \\
\hline $10 N=013 N-02$ & DAC & 1 & RUSH & SPRINGS & SANDSTONE & & 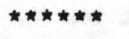 & 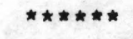 & $12=01-75$ & 75.03 \\
\hline $010 N-013 N=03$ & $\operatorname{COO}$ & 1 & RUSH & SPRINGS & SANUSTUNE & $12-17-74$ & 87.49 & $\| \star \star \star \star \star \star *$ & & $\star \star$ \\
\hline $10 N-013 N=09$ & DBC & 1 & RUSH & SPRINGS & SANOSTONE & $12-17-74$ & 78.35 & -4.95 & $12-02-75$ & 83.30 \\
\hline $10 N-013 m-11$ & $O C A$ & 1 & RUSH & SPRINGS & SANOSTONE & $12-17-74$ & 75 & 1.99 & $12-02-75$ & 73.91 \\
\hline $010 N-013 m-12$ & 080 & 1 & FUSH & SPRINGS & SANDSTUNE & $12-17-74$ & 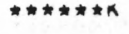 & $\star \star \star \star \star \star \star \star$ & $12-01-75$ & th \\
\hline $010 N=013 m-14$ & CAU & 1 & RUSH & SPRINGS & SANDSTONE & $12-17-74$ & 75.09 & 3.94 & $12-01-75$ & 71.15 \\
\hline $010 N-013 n=15$ & $A C A$ & 1 & RUSA & SPRINGS & SANOSTUNE & $12-16-74$ & 76.09 & 2.01 & $12-02-75$ & 76.08 \\
\hline $010 N-013 N=17$ & $\operatorname{cco}$ & 1 & RUSH & SPKINGS & SANUSTONE & $12-17-74$ & 19 & 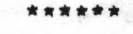 & $12-02-75$ & * \\
\hline $010 N-013 m-22$ & $\triangle A C$ & 1 & RUSH & SPHINGS & SANOSTUNE & $12-17-74$ & $\star \star \star \star$ & $\hbar \star \star \star \star \star \star \star *$ & $12=01-75$ & 60.61 \\
\hline $010 N=013 N=20$ & $A \Delta O$ & 1 & RUSH & SPRINGS & SANUSTUIVE & $12-17-74$ & $\star$ & 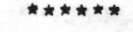 & $12=01-75$ & $\star \star \star x$ \\
\hline $010 N=013 N=27$ & $A C A$ & 1 & RUST & SPRINGS & SANUSTDNE & $12-17-74$ & 48.87 & 2.64 & $12-02-75$ & 46.23 \\
\hline $010 N-013 N-30$ & $\Delta \theta \Delta$ & 1 & RUSH & SPRIVGS & SANUSTONE & & $\star \star \star \star \star \star \star \star$ & 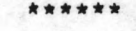 & $12=02-75$ & 38.08 \\
\hline $010 N-013 n-32$ & $\Delta C y$ & 1 & RUSH & SPRINGS & SANDSTUNE & $12-17-74$ & **k & $\star \star \star \star \star \star \star \star \Delta$ & $12-02-75$ & 8.43 \\
\hline $010 N-013 n-30$ & $\triangle O C$ & 1 & RUSH & SPRINGS & SANDSTONE & $12-17-74$ & 05.19 & -0.85 & $12-01-75$ & 00.04 \\
\hline $010 N=014 m=06$ & $c C_{0}$ & 1 & RUSH & SPRINGS & SANOSTUNE & & * & 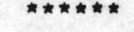 & $12=03-75$ & 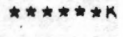 \\
\hline $01 \cup N-014 N-11$ & $50 \mathrm{C}$ & 1 & RUSH & SPRINGS & SANUSTUNE & & 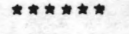 & 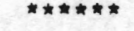 & $12=03-75$ & 47.58 \\
\hline $010 N-014 n-12$ & Acio & 1 & RUSH & SPRINGS & SANDSTUNE & & 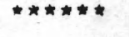 & 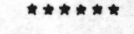 & $12-03-75$ & 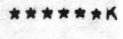 \\
\hline $10 N-014 W=30$ & Dü & 1 & RUSH & SPRINGS & SANUSTONE & & 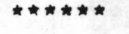 & 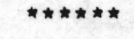 & $12=03-75$ & $\hbar \star \star \star \star * \star \star k$ \\
\hline
\end{tabular}


Table 1.-Water levels and water-level changes--Continued.

CADOD COUNTY

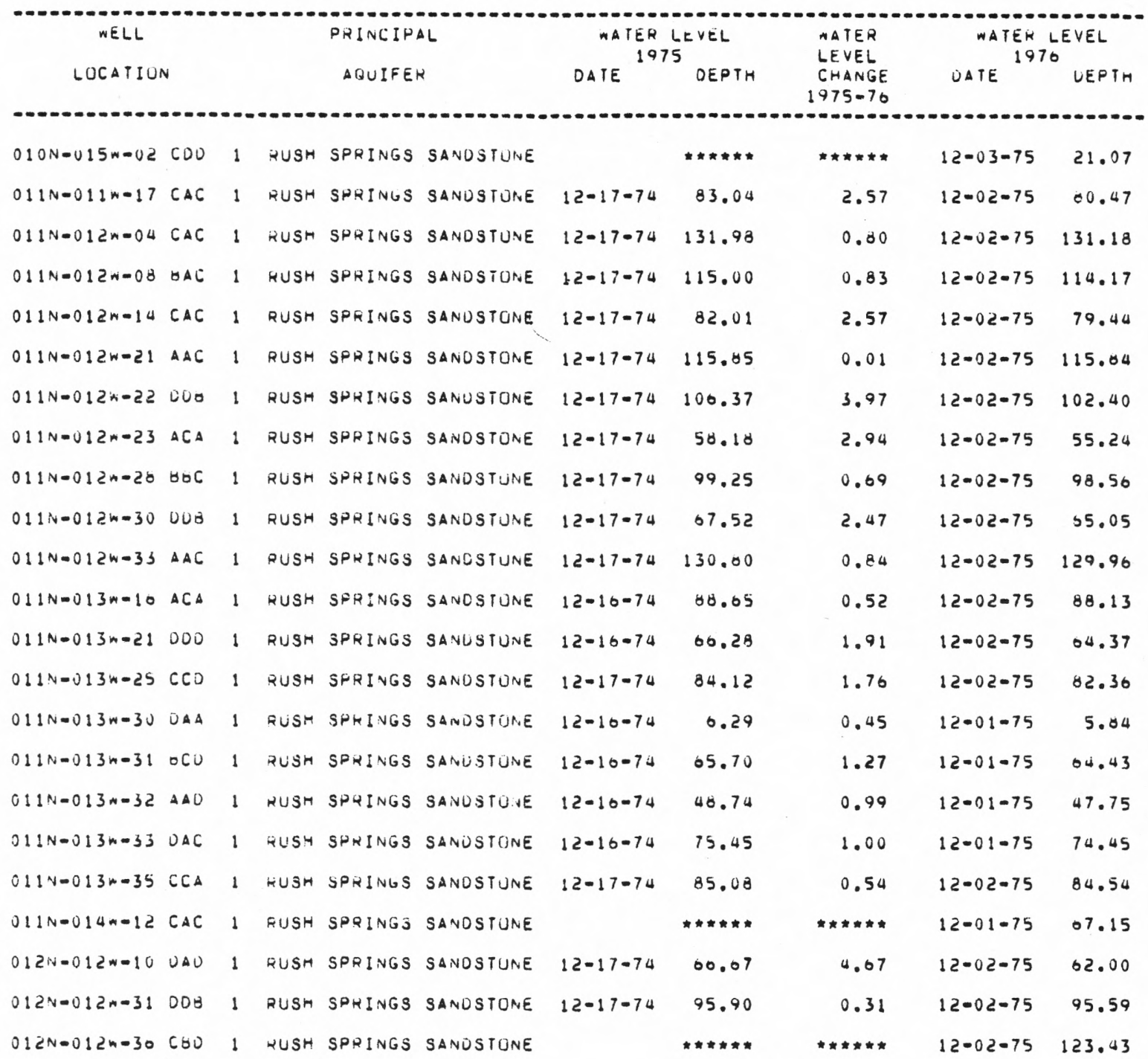


Table 1.-Water levels and water-level changes--Continued.

CADDO CUUNTY

\begin{tabular}{|c|c|c|c|c|c|c|c|c|c|}
\hline WÉLL & & & PKINCIPA & & $\begin{array}{r}\text { WATER } \\
197\end{array}$ & & $\begin{array}{l}\text { WATER } \\
\text { LEVEL }\end{array}$ & $\begin{array}{r}\text { WATER } \\
19\end{array}$ & 年 \\
\hline LOCATIUN & & & $A$ AUIFER & & DATE & DEPTH & $\begin{array}{r}\text { CHANGE } \\
1975-76\end{array}$ & DATE & DEPIH \\
\hline $012 N=013 N-21 C A C$ & 1 & RUSH & SPKINGS & SANOSTUNE & $12-17-74$ & 70.47 & 0.14 & $12-02-75$ & 70.33 \\
\hline $012 N-013 m-27 \quad \forall A C$ & 1 & RUSH & SPRINGS & SANOSTUNE & $12-17-74$ & 64.00 & -0.78 & $12-02-75$ & 65.58 \\
\hline
\end{tabular}


Table 1.-Water ievels and water-level changes--Continued.

CIMARRUN COUNTY

\begin{tabular}{|c|c|c|c|c|c|c|c|c|}
\hline \multirow{2}{*}{\multicolumn{2}{|c|}{$\begin{array}{l}\text { WELL } \\
\text { OCATION }\end{array}$}} & \multirow{2}{*}{\multicolumn{2}{|c|}{$\begin{array}{l}\text { PRINCIPAL } \\
\text { AOUIFER }\end{array}$}} & \multicolumn{2}{|c|}{$\begin{array}{l}\text { WATER LEVEL } \\
1975\end{array}$} & \multirow{2}{*}{$\begin{array}{l}\text { NATER } \\
\text { LEVEL } \\
\text { CHANGE } \\
1975-76\end{array}$} & \multicolumn{2}{|c|}{$\begin{array}{l}\text { WATEK LEVEL } \\
1970\end{array}$} \\
\hline & & & & DATE & UEPTH & & DATE & UEPTH \\
\hline $001 N-001 E-10$ & $\triangle A O$ & 1 & MURRISUN FURMAIIUN & $01-22-75$ & 105.14 & -1.30 & $0:-13-70$ & 166.44 \\
\hline $001 N-C 01 E-15$ & $36 \overline{9}$ & 1 & $\begin{array}{l}\text { DAKUTA SANUSTONE } \\
\text { CHEYENNE SANUSTONE }\end{array}$ & $01-22-75$ & 184.38 & 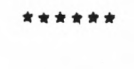 & $01-14-76$ & $\hbar \hbar$ \\
\hline $001 N=001 E-20$ & $D C C$ & 1 & CHEYENNE SANUSTONE & $01-22-75$ & 187.28 & -0.70 & $01-14-76$ & 188.04 \\
\hline $001 N=001 E-25$ & CoO & 1 & CMEYENNE SANUSTUNE & $01-22-75$ & 171.91 & 0.12 & $01-14-76$ & 171.19 \\
\hline $001 N-001 E-33$ & $\triangle C O$ & 1 & $\begin{array}{l}\text { DAKUTA SANOSTONE } \\
\text { CHEYENIVE SANDSTONE }\end{array}$ & $01-22-75$ & 187.39 & -2.49 & $01-14-76$ & 189.08 \\
\hline $001 N-001 E-35$ & AHC & 1 & CHEYENNE SARDSTONE & $01-22 \cdot 75$ & $10 \overline{.36}$ & -1.98 & $01-14-76$ & 171.34 \\
\hline $001 N=002 E=05$ & UDO & 1 & ChEYENAE SANUSTONE & $01-22-75$ & 117.80 & 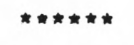 & $01-14=76$ & $\star \star \star \star \star \star \star$ \\
\hline $001 N=002 E=07$ & OAA & 1 & CHEYENNE SANDSTONE & $01-22-75$ & 143.34 & -10.56 & $01-14=76$ & 153.90 \\
\hline $001 N-002 E=09$ & $\forall \subset 5$ & 1 & DAKOTA SANOSTONE & $01-22-75$ & 133.10 & -4.68 & $01-14-76$ & 137.78 \\
\hline $001 N=002 E-10$ & $\$ 00$ & 1 & CHEYENNE SANUSTONE & $01-22-75$ & 110.28 & 1.81 & $01-14=70$ & 108.47 \\
\hline $001 N-002 E-12$ & Abó & 1 & CHEYENNE SANOSTONE & $01-22-75$ & 90.15 & -4.64 & $01-14=76$ & 100.79 \\
\hline $001 N-002 E-13$ & $\forall B C$ & 1 & CHEYENNE SANDSTOME & $01-20-75$ & 105.72 & 5.12 & $01-14=76$ & 100.60 \\
\hline $001 N=002 E-16$ & $B D C$ & 1 & CAEYENNE SARIOSTUNE & $01-22-75$ & 84.35 & -11.06 & $01-14=76$ & 95.41 \\
\hline $001 N=002 E-24$ & $\operatorname{Cos}$ & 1 & CHEYENNE SANUSTONE & $01-20-75$ & 118.00 & -3.10 & $01-14-76$ & 121.10 \\
\hline $001 N=002 E-20$ & $B \Delta U$ & 1 & CHEYENIE SANUSTONE & $01-20-75$ & 123.75 & -3.57 & $01-14-76$ & 127.32 \\
\hline $001 N=002 E-27$ & $O C C$ & 1 & DAXUTA SANOSTONE & $01-22-75$ & 89.93 & -0.48 & $01-14-76$ & 90.41 \\
\hline $001 N-002 E-29$ & $00 \mathrm{~A}$ & 1 & CMEYENNE SANUSTUNE & & 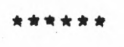 & 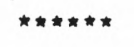 & $01-14=70$ & 113.59 \\
\hline $001 N-002 E-30$ & $O C C$ & 1 & OGALLALA FORMATION & $01-22-75$ & 142.93 & -1.00 & $01-14-70$ & 143.99 \\
\hline $001 N=0 \cup 3 E=00$ & $A O B$ & 1 & $\begin{array}{l}\text { DAROTA SANUSTUNE } \\
\text { CMEYENAE SANDSTUNE }\end{array}$ & $01-20-75$ & 82.79 & 0.17 & $01-14-76$ & 82.62 \\
\hline $001 N=003 t=09$ & 000 & 1 & UGaLlaLa FuRMatiUn & $01-20-75$ & 99.89 & -0.43 & $01-14-70$ & 100.32 \\
\hline $001 N=003 E-14$ & $\mathrm{OCO}$ & 1 & $\begin{array}{l}\text { OAKUTA SANUSTONE } \\
\text { CHEYENNE SANDSTUNE }\end{array}$ & $01-20-75$ & 103.44 & 1.59 & $01-14-76$ & 101.85 \\
\hline
\end{tabular}


Table 1.-Water levels and water-level changes--Continued.

CIMARRUN CUUNTY

\begin{tabular}{|c|c|c|c|c|c|c|c|c|}
\hline \multirow{2}{*}{$\begin{array}{c}\text { WELL } \\
\text { LUCATIUT }\end{array}$} & & \multirow{2}{*}{\multicolumn{2}{|c|}{$\begin{array}{l}\text { PRINCIPAL } \\
\text { AUUIFER }\end{array}$}} & \multicolumn{2}{|c|}{$\begin{array}{l}\text { NATER LEVEL } \\
1975\end{array}$} & \multirow{2}{*}{$\begin{array}{l}\text { NATER } \\
\text { LEVEL } \\
\text { CHANGE } \\
1975=76\end{array}$} & \multicolumn{2}{|c|}{$\begin{array}{c}\text { WATER LEVEL } \\
1976\end{array}$} \\
\hline & & & & DATE & DEPTH & & DATE & DEPTH \\
\hline $001 N=003 E-17$ & $\mathrm{CaC}$ & 1 & $\begin{array}{l}\text { DAKOTA SANUSTUNE } \\
\text { CAEYENNE SANUSTUNE }\end{array}$ & $01-22-75$ & 103.51 & 0.18 & $01-14-76$ & 103.33 \\
\hline $001 N=003 E-19$ & $\mathrm{cco}$ & 1 & $\begin{array}{l}\text { OAKOTA SANDSTONE } \\
\text { CHEYENNE SANOSTONE }\end{array}$ & $01-20-75$ & 103.10 & -5.35 & $01-14-76$ & 108.45 \\
\hline $001 N-003 E-25$ & $\Delta b 8$ & 1 & $\begin{array}{l}\text { DAKUTA SANUSTONE } \\
\text { CHEYENNE SANUSTONE }\end{array}$ & $01-20-75$ & 92.64 & -5.13 & $01-14-76$ & 97.77 \\
\hline $001 N=003 E-29$ & $\operatorname{ccs}$ & 1 & $\begin{array}{l}\text { OAKOTA SANOSTONE } \\
\text { CHEYENNE SANDSTONE }\end{array}$ & $01-20-75$ & 177.35 & 10.83 & $01-14-76$ & 100.52 \\
\hline $001 N-003 E-32$ & CAA & 1 & OGALLALA FURMATION & $01-2 v-75$ & 61.26 & 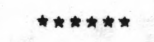 & $01-14-76$ & $\# \star \star \star \star \star \star \hbar K$ \\
\hline $001 N-003 k-33$ & UCC & $!$ & UGALLALA FURMATION & $01-20-75$ & 37.03 & -2.84 & $01-14-76$ & 39.87 \\
\hline $001 N=003 E-35$ & CAA & 1 & $\begin{array}{l}\text { DAKUTA SANUSTONE } \\
\text { CHEYENNE SANDSTUNE }\end{array}$ & $01-20-75$ & 79.23 & -1.14 & $01-14-76$ & 80.37 \\
\hline $001 N-004 E-09$ & 000 & 1 & DGALLALA FUHMATION & $01-20-75$ & 63.92 & 1.55 & $01-14-76$ & 62.37 \\
\hline $001 N-000 E-20$ & $A O C$ & 1 & DGALLALA FURMATION & $01-20-75$ & 54.20 & -1.44 & $01-14-76$ & 55.08 \\
\hline $001 N=004 E-30$ & טDo & 1 & UGALLALA FURMAIION & $01-20-75$ & $\hbar \hbar \star \hbar \star \star \hbar K$ & $\neq * \star \star \star * \star$ & $01-14=76$ & $\star \star \star \star \star \star \star \star M M$ \\
\hline $001 N=005 E-25$ & $\operatorname{CBO}$ & 1 & OGALLALA FUKMATION & $01-20-75$ & 01.45 & -2.13 & $01-14-76$ & 03.58 \\
\hline $001 N-005 E-27$ & Dod & 1 & OGALLALA FUYMATIUN & $01-20-75$ & 05.50 & $\star \star \star \star \star \star * \star$ & $01-14-76$ & 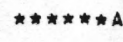 \\
\hline $001 N-005 E-32$ & $00 \mathrm{~A}$ & $!$ & DGALLALA FORMATILIV & $01-20-75$ & 07.75 & -0.27 & $01-14=76$ & 68.02 \\
\hline $001 N-005 E-34$ & cou & 1 & UGALLALA FORMATIUN & $01-20-75$ & 80.05 & -0.69 & $01-14-76$ & 80.74 \\
\hline $001 N=005 E-35$ & DoC & 1 & UGALLALA FUKMATIUN & $01-20-75$ & 60.53 & -1.24 & $01-14-76$ & 69.77 \\
\hline OUIN-UOGE-OS & $d o A$ & 1 & UGALLALA FURMATION & $01-20-75$ & 102.89 & -0.04 & $01-14-76$ & 102.93 \\
\hline $001 N=0005=20$ & $\operatorname{cCc}$ & 1 & OGALLALA FURMATIUN & $01-20-75$ & 109.37 & 0.00 & $01-14=70$ & 103.37 \\
\hline $001 N-007 E-10$ & DCC & 1 & OGALLALA FORMATION & $01-20-75$ & 89.60 & 1.42 & $01-14=76$ & 88.24 \\
\hline $001 N-007 E-28$ & $\forall A U$ & 1 & DOCKUM GRUUP & $01-20-75$ & 192.97 & -0.748 & $01-14-76$ & 193.715 \\
\hline $001 N-008 E=03$ & $\Delta \forall 0$. & 1 & OGALLALA FORMATIUN & $01-21-75$ & 301.54 & 0.71 & $01-14-76$ & 300.83 \\
\hline
\end{tabular}


Table 1.-Water levels and water-level changes--Continued.

CIMARRON CUUNTY




Table 1.-Water levels and water-level changes--Continued.

GIMARRUN COUNTY

\begin{tabular}{|c|c|c|c|c|c|c|c|c|c|}
\hline WEL & & & p & PAL & $\begin{array}{r}\text { MATER } \\
197\end{array}$ & 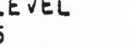 & $\begin{array}{l}\text { WATER } \\
\text { LEVEL }\end{array}$ & $\begin{array}{l}\text { WATEK } \\
\text { IGT }\end{array}$ & EV VEL \\
\hline LOCATION & & & $A G$ & UIFER & DATE & DEPTH & $\begin{array}{r}\text { CHANGE } \\
1975-76\end{array}$ & DATE & DEPTH \\
\hline $002 N-007 E-25$ & CBo & 1 & DGALLALA & FURMATIUN & $01-21-75$ & 187.22 & -1.13 & $01-14-76$ & 188.35 \\
\hline $002 N=007 E-26$ & $\triangle A C$ & 1 & OGALLALA & FURMATION & $01-21-75$ & 230.98 & -4.39 & $01-14-76$ & 235.37 \\
\hline $002 N-008 E-17$ & $\triangle B A$ & 1 & OGALLALA & FURMATIUIV & $01-21-75$ & 270.90 & -2.35 & $01-14-76$ & 273.25 \\
\hline $002 N-008 E-19$ & 068 & 1 & OGALLALA & FURMATION & $01-21-75$ & 238.81 & -0.99 & $01-14-76$ & 239.80 \\
\hline $002 N-00 \triangle E-20$ & $A B A$ & 1 & OGALLALA & FURMATION & $01-21-75$ & 274.40 & 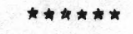 & $01-14-76$ & $\star \star \star \star \star \star \star \star M M$ \\
\hline $002 N=008 E-22$ & $\triangle B C$ & 1 & OGALLALA & FORMATIUN & $01-21-75$ & 282.60 & -7.12 & $01-14-76$ & 289.72 \\
\hline $002 N=008 E-20$ & $A \theta \forall$ & 1 & OGALLALA & FURMATION & $01-21-75$ & 306.40 & -3.20 & $01-14-76$ & 300.60 \\
\hline $002 N=008 E-36$ & 388 & 1 & DGALLALA & FURMATION & $01-21-75$ & $\star \star \star \star \star \star \star \star k K$ & 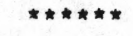 & $01-14-76$ & $\star \star \star \star \star \star \star M$ \\
\hline $002 N-009 E-02$ & $A C D$ & 1 & OGALLALA & FURMATION & $01-22-75$ & 116.20 & -3.98 & $01-14-76$ & 120.18 \\
\hline $002 N-009 E-33$ & $A B \theta$ & 1 & UGALLALA & FORMATILN & $01-22=75$ & 272.20 & -4.13 & $01-14-76$ & 276.33 \\
\hline $003 N-002 E-28$ & CCC & 1 & UGALLALA & FORMATIUN & $01-21-75$ & 19.95 & 3.50 & $01-13-76$ & 16.45 \\
\hline $003 N-004 E-11$ & CAC & 1 & OGALLALA & FUFMATIUN & $01-22-75$ & 160.10 & -0.71 & $01-13-76$ & 160.81 \\
\hline $003 N=004 E-17$ & 884 & 1 & OGALLALA & FORMATION & $01-22-75$ & 126.40 & -0.99 & $01-13-70$ & 127.39 \\
\hline $003 N-004 E-30$ & CAA & 1 & OGALLALA & FORMAIION & $01-22-75$ & $\star \star \star \star \star \star \star A$ & $\# \star \star \star \star \star \star$ & $01-15-76$ & 157.69 \\
\hline $003 N-004 t-33$ & $\forall \subset c$ & 1 & OGALLALA & FORMATION & $01-22=75$ & 130.96 & 4.08 & $01-15=76$ & 126.88 \\
\hline $003 N=005 E=03$ & $B C C$ & 1 & OGALLALA & FURMATIUN & $01-20-75$ & $\star \star \star \omega * \star \star \star k K$ & $\hbar \star \star \star \star \star * *$ & $01-14=76$ & $\star \star M$ \\
\hline $003 N=005 E=05$ & UAA & 1 & IJGALLALA & FURMATIUN & $01-22-75$ & 214.95 & 2.23 & $01-15-70$ & 212.72 \\
\hline $003 N=005 E=09$ & $\forall \subset 0$ & 1 & IJGALLALA & FURMATION & $01-22-75$ & 221.37 & -1.21 & $01-15-76$ & 222.58 \\
\hline $003 N=005 E-11$ & $\operatorname{ccc}$ & 1 & OGALLALA & FURMATIUN & $01-20-75$ & 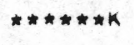 & $\star \star * * \star * *$ & $01-14=76$ & 184.25 \\
\hline $003 N=005 E-12$ & $A C C$ & 1 & UGALLALA & FORMATIUN & $01-20-75$ & 159.94 & -2.21 & $01-14-70$ & 102.15 \\
\hline $003 N-005 E-10$ & DSA & 1 & OGALLALA & FURMATIUN & $01-22-75$ & $\geqslant * * * * * k$ & $* * * *$ & $01-13-76$ & 228.88 \\
\hline $003 N-005 E-18$ & 808 & 1 & DGALLALA & FORMATIUN & $01-22-75$ & 180.40 & 1.60 & $01-13-76$ & 104.80 \\
\hline $003 N-005 E-23$ & $\forall 80$ & 1 & UGALLALA & FORMATIUN & $01-21-75$ & 190.90 & 9.32 & $01-13-76$ & 189.58 \\
\hline
\end{tabular}


Table 1.-Water levels and water-level changes--Continued.

CIMARRION COUNTY

\begin{tabular}{|c|c|c|c|c|c|c|c|c|c|}
\hline \multirow{2}{*}{\multicolumn{2}{|c|}{$\begin{array}{c}\text { WELL } \\
\text { LUCATION }\end{array}$}} & \multirow{2}{*}{\multicolumn{3}{|c|}{$\begin{array}{l}\text { PRINCIPAL } \\
\text { AQUIFER }\end{array}$}} & \multicolumn{2}{|c|}{$\begin{array}{l}\text { WATER LEVEL } \\
1975\end{array}$} & \multirow{2}{*}{$\begin{array}{l}\text { WATEN } \\
\text { LEVEL } \\
\text { CHANGE } \\
1975-76\end{array}$} & \multicolumn{2}{|c|}{$\begin{array}{c}\text { WATEN LEVEL } \\
1976\end{array}$} \\
\hline & & & & & DATE & DEPTH & & DAIE & UEPTH \\
\hline $003 N-005 E-30$ & $U C A$ & 1 & UEALLALA & FORMATIÜN & $01-22-75$ & $\star \star \star \star \star \star \star \hbar k$ & 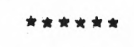 & $01-15-76$ & 152.54 \\
\hline $003 N-006 E-01$ & $A \triangle C$ & 1 & UGALLALA & FURMATIUN & $01-22-75$ & 103.13 & -1.77 & $01-14=70$ & 104.90 \\
\hline $003 N-000 E-04$ & 880 & $!$ & OGALLALA & FURMATIUN & $01-22-75$ & 155.13 & -0.77 & $01-14=70$ & 163.90 \\
\hline $003 N=000 E=05$ & $\forall A D$ & 1 & UGALLALA & FURMATIUN & $01-22-75$ & $\star \star * \star * \star * \star k$ & 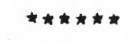 & $01-14=76$ & 188.35 \\
\hline $003 N=006 E-08$ & $\mathrm{Cos}$ & 1 & UGALLALA & FORMATION & $01-20-75$ & 152.62 & -4.71 & $01-14-70$ & 157.33 \\
\hline $003 N=006 E=09$ & $B C C$ & 1 & OGALLALA & FORMATIUN & $01-20-75$ & 146.21 & -3.41 & $01-14-76$ & 149.02 \\
\hline $003 N=006 E-10$ & $c \subset 0$ & 1 & OGALLALA & FORMATIUN & $01-22-75$ & 144.00 & $\star \star \star * * * *$ & $01-14-76$ & $\hbar * \hbar \# \approx m M$ \\
\hline $0 \cup 3 N-1) 00 E-14$ & $\operatorname{A\theta O}$ & 1 & DGALLALA & FURMATION & $01-22-75$ & 115.76 & 1.32 & $01-14-70$ & 114.44 \\
\hline $003 N=000 E=15$ & $D C A$ & 1 & OGALLALA & FURMATIUN & $01-22-75$ & 120.32 & -3.83 & $01-14-76$ & 130.15 \\
\hline $003 N-000 E-16$ & 600 & 1 & OGALLALA & FURMATIUN & $01-20-75$ & 117.00 & -11.58 & $01-14-76$ & 129.26 \\
\hline $003 N-007 k=09$ & 800 & 1 & OGALLALA & FURMATIUN & $01-22-75$ & 31.33 & 0.23 & $01-14-76$ & 31.10 \\
\hline $003 N-007 E-32$ & $B A A$ & 1 & UGALLALA & FORMATION & $01-21-75$ & 138.04 & -0.59 & $01-14=76$ & 138.03 \\
\hline $003 N-007 E-33$ & DUC & 1 & UGALLALA & FURMATIUN & $01-21-75$ & 136.90 & -0.21 & $01-14-76$ & 137.11 \\
\hline $003 N-009 E-13$ & $A E O$ & 1 & OGALLALA & FURMATIUN & $01-22-75$ & 195.92 & 5.08 & $01-14-70$ & 190.84 \\
\hline $003 N=009 E-15$ & $\mathrm{CoC}$ & 1 & DGALLALA & FURMATIUN & $01-22=75$ & 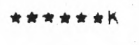 & 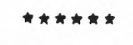 & $01-14-70$ & 250.48 \\
\hline $004 N=004 E-23$ & soo & -1 & UGALLALA & FURMATIUN & $01-22-75$ & $\# * \# \# \# \# \Delta$ & $\star \star \star \star * \star * *$ & $01-15-70$ & 93.13 \\
\hline $004 N=004 E-20$ & $E C A$ & 1 & OGALLALA & FORMATIUN & $01-22-75$ & 207.90 & -4.26 & $01-15-76$ & 212.16 \\
\hline$O O L N=004 E-30$ & $\triangle A C$ & 1 & OGALLALA & FUKMATIUN & $01-2 \bar{c}-75$ & 103.80 & -2.10 & $01-14-70$ & 185.90 \\
\hline $004 N-00 S E-15$ & cot & 1 & CHEYENNE & SANOSTONE & $01-20-75$ & $\star \star \star \star \star \star \star \star * k$ & $\star \star \star * \star * * *$ & $01-14-76$ & 122.02 \\
\hline $004 N=005 E-24$ & $\dot{v \theta b}$ & 1 & OGALLALA & FURMATIUN & $01-20-75$ & $\star \star \star \star \star \star \star \star x$ & x 2 - & $01-14-76$ & 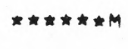 \\
\hline $004 N-1005 E-31$ & $C C O$ & 1 & OGALLALA & FURMATION & $01-22-75$ & 187.53 & -2.62 & $01-14=76$ & 190.15 \\
\hline $004 N=005 E-32$ & $A A A$ & 1 & OGALLALA & FUNMATIUN & $01-22-75$ & 180.79 & 8.74 & $01-14-70$ & 180.05 \\
\hline OOUN-UOSE-30 & $A C \theta$ & 1 & OGALLALA & FGKMATIUN & $01-20-75$ & 189.55 & 0.35 & $01-14=76$ & 149.20 \\
\hline
\end{tabular}


Table 1.-Water levels and water-level changes--Continued.

CIMARRUN COUNTY

\begin{tabular}{|c|c|c|c|c|c|c|c|c|}
\hline WELL & & & PRINCIPAL & $\begin{array}{l}\text { WATER } \\
197\end{array}$ & LEVEL & $\begin{array}{l}\text { NATER } \\
\text { LEVEL }\end{array}$ & $\begin{array}{l}\text { WATER } \\
197\end{array}$ & EVEL \\
\hline . LUCATIUA & & & $\triangle Q U I F E R$ & DATE & DEPTH & $\begin{array}{r}\text { CHANGE } \\
1975-76\end{array}$ & OATE & DEPTH \\
\hline $004 N=000 E-02$ & CDO & 1 & OGALLALA FURMATION & $01-22-75$ & $\star \star \star \star \star \star \hbar F$ & 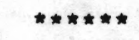 & $01-14-76$ & $\star \star$ \\
\hline $004 N-100 E=09$ & $A C B$ & 1 & DOCKUM GROUP & $01-22-75$ & 213.29 & -3.76 & $01-14-70$ & 217.05 \\
\hline $004 N-006 E-17$ & cuc & 1 & DUCKUM GROUP & $01-20-75$ & 222.41 & -2.59 & $01-14-76$ & 225.00 \\
\hline $004 N=006 E-21$ & $C C D$ & 1 & OGALLALA FURMATION & $01-22-75$ & 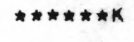 & $\star * * * \star * \star$ & $01-14-76$ & $\star \star M$ \\
\hline $004 N=006 c-22$ & $C \forall 0$ & 1 & OGALLALA FORMATION & $01-22-75$ & 174.32 & -25.13 & $01=14=76$ & 199.45 \\
\hline $004 N=000 E-23$ & $O A G$ & 1 & OGALLALA FORMATIUN & $01-22-75$ & 148.30 & 0.00 & $01-14-76$ & 148.30 \\
\hline $004 N=006 E-25$ & $8 \Delta C$ & 1 & JGALLALA FURMATIUN & $01-22-75$ & 130.31 & -19.46 & $01-14-76$ & 149.77 \\
\hline $004 N-006 E-20$ & $\cos$ & 1 & UGALLALA FORMATION & $01-22-75$ & $\star \star \star * * * * A$ & $* * * * * *$ & $01-14=76$ & 142.32 \\
\hline$O U 4 N-0 O O E-30$ & UCC & 1 & OGALLALA FURMATIUN & $01-20-75$ & 194.45 & -5.10 & $01-14-76$ & 199.55 \\
\hline $00 \backsim N=007 E=01$ & DAA & 1 & OGALLALA FORMATION & $01-21-75$ & 190.00 & 2.60 & $v 1-14-76$ & 193.40 \\
\hline $004 N=007 E=03$ & $\operatorname{CAA}$ & 1 & DGALLALA FUKMAIIUN & $01-22-75$ & 189.59 & 0.34 & $01-14-76$ & 189.25 \\
\hline $004 N=007 E-07$ & $A \Delta B$ & 1 & UGALLALA FOEMATION & $01-22-75$ & 210.21 & -9.63 & $01-14-76$ & 219.84 \\
\hline $004 N=007 t=09$ & CBO & 1 & OGALLALA FORMATIUN & $01-22-75$ & 200.70 & -9.03 & $01-14-76$ & .79 \\
\hline $004 N-007 E-15$ & $\triangle B C$ & 1 & OGALLALA FORMATIUN & $01-22-75$ & $\star \star \star \star \star \star \star \star \star K$ & $\star \star \star \star \star \star \star \star *$ & $01-14-76$ & 150 \\
\hline $004 N-007 E-16$ & $A C C$ & 1 & OGALLALA FORMATION & $1-22-75$ & 150.13 & 3.70 & $01-14-70$ & 146.43 \\
\hline $004 N=007 E-18$ & $\cos \theta$ & 1 & UGALLALA FORMATION & $01-22-75$ & 164.40 & -0.35 & $01-14-76$ & 172.75 \\
\hline $004 N=007 E-32$ & ن४⿻ & 1 & UGALLALA FORMATIUN & $01-22-75$ & $\star \star \star \star \star \star \star \star \hbar k$ & 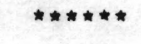 & $01-14-76$ & 96.50 \\
\hline $004 N=00$ dE $=05$ & $A A B$ & 1 & UGALLALA FURMATION & $01-20-75$ & 102.82 & -2.73 & $01-14-70$ & 185.55 \\
\hline $004 N=009 E-11$ & ADA & 1 & DUCKUM GROUP & $01-20-75$ & 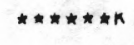 & 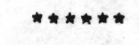 & $01-14-76$ & 219.28 \\
\hline $005 N=007 E-35$ & $\operatorname{cco}$ & 1 & UGALLALA FORMATION & $01-21-75$ & $\star \star \star \star \star * * \hbar \pi$ & $m \star \star \star \star \star \star *$ & $01-13-76$ & 228.40 \\
\hline $005 N=009 E=05$ & $A \Delta A$ & 1 & OGALLALA FURMAIIUN & $01-20-75$ & 178.96 & $\# \star \star \star \star \star * *$ & $01-14-76$ & $* * k$ \\
\hline $005 N=009 E-22$ & $\triangle B A$ & 1 & UGALLALA FORMATIUN & $01-21-75$ & 184.60 & 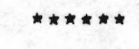 & $01-14-70$ & $* \star \star M$ \\
\hline $005 N-009 E-30$ & $\operatorname{CBC}$ & 1 & UGALLALA FURMATIUN & $01-22-75$ & 199.41 & -0.27 & $01-14=76$ & 199.68 \\
\hline
\end{tabular}


Table 1.--Water levels and water-level changes--Continued.

CIMARRON COUNTY

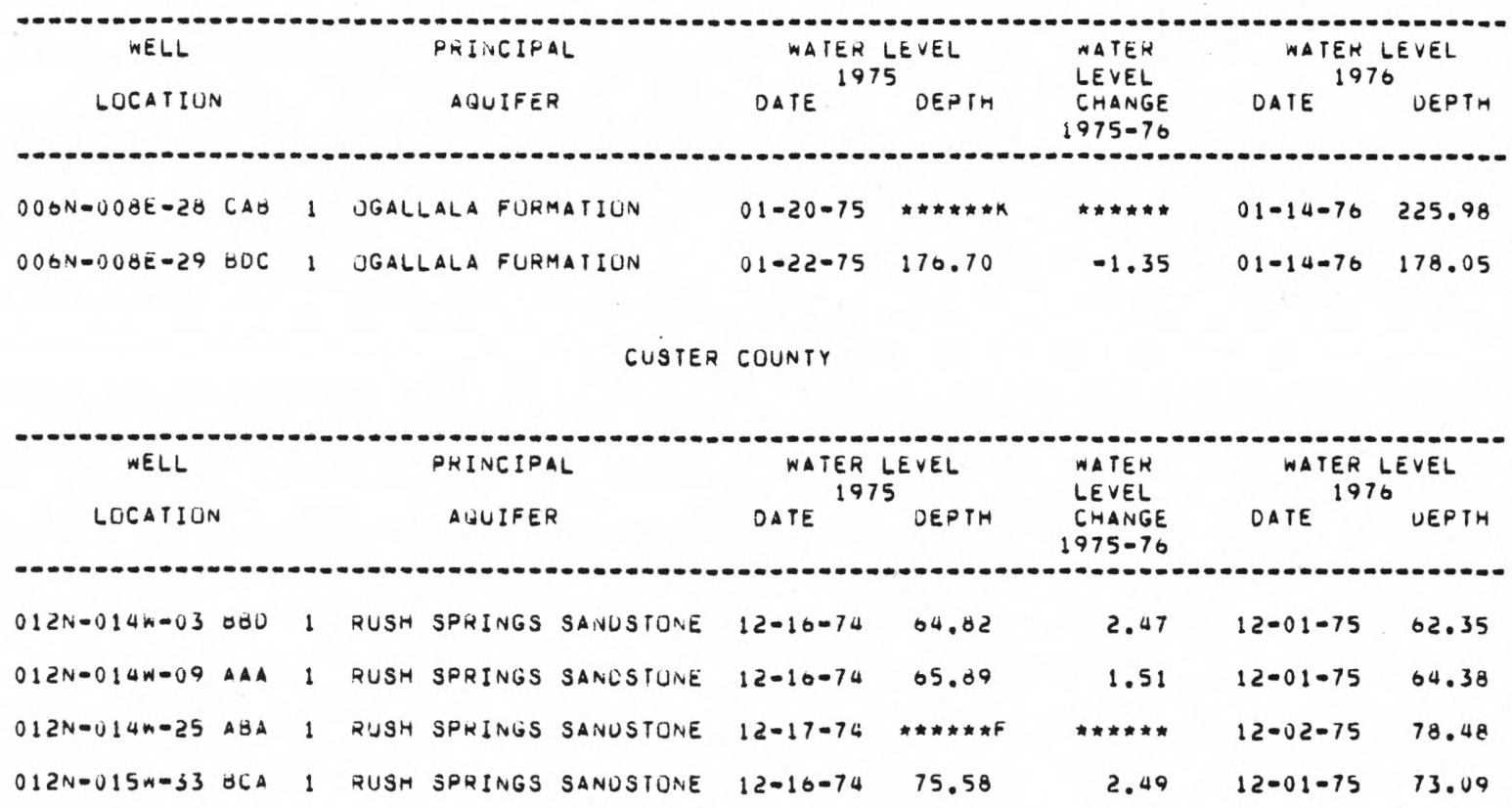

ELLIS COUNTY

\begin{tabular}{|c|c|c|c|c|c|c|c|c|}
\hline \multirow{2}{*}{\multicolumn{2}{|c|}{$\begin{array}{c}\text { WELL } \\
\text { LOCATIUN }\end{array}$}} & & \multirow{2}{*}{$\begin{array}{l}\text { PRINCIPAL } \\
\text { AQUIFER }\end{array}$} & \multicolumn{2}{|c|}{$\begin{array}{l}\text { WA TER LEVEL } \\
1975\end{array}$} & \multirow{2}{*}{$\begin{array}{l}\text { WATER } \\
\text { LEVEL } \\
\text { CHANGE } \\
1975-76\end{array}$} & \multicolumn{2}{|c|}{$\begin{array}{c}\text { WATEK LEVEL } \\
1976\end{array}$} \\
\hline & & & & DATE & DEPTH & & DATE & DEPTH \\
\hline $01-v-121 a-155$ & 384 & 1 & TEUMACE DEDUSITS & & $\star \star \star \star \star \star \star \star$ & $\star \star * \star * \star *$ & $12-12-75$ & 132.03 \\
\hline ) $14-024 \ldots-11$ & ¿C. & 1 & TEADACE VEDUSITS & & $\star * \star * * *$ & $\star \star \star \star \star \star \star$ & $12-12-75$ & 165.73 \\
\hline$\pi 1+v-1025,-1 j n$ & UA & 1 & TEKRACE LEPIJSITS & $03-19-75$ & 110.79 & $\star \star \star \star \star \star \star$ & & 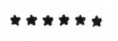 \\
\hline 1) $20 N-024 n-33$ & $A \cup C$ & $!$ & TERRACE UERUSATS & & $\star \star \star \star \star \star \star$ & $\star \star \star \star \star \star \star \star *$ & $12-12-75$ & 117.55 \\
\hline $020 N-020 m-15$ & DCC & 1 & TERKACE DEPUSITS & & $\pitchfork \star \star \star \star \star$ & 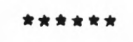 & $02-10-70$ & 107.50 \\
\hline $21 N=024 n-10$ & ט甘ם & 1 & TERRACE UEPOSITS & & 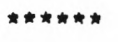 & $\star \star \star \star \star \star \star$ & $02-10-76$ & 9.80 \\
\hline $21 N-025 n-24$ & CCC & 1 & TERRACE DEPUSITS & & 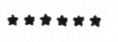 & $\hbar \star \star \star \star \star \star \star$ & $02-10-10$ & 39.52 \\
\hline $21 N=025 w-34$ & CBO & 1 & TERRACE DEPOSITS & & 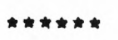 & $\star \star \star \star \star \star \star \star$ & $02-10-76$ & 02.59 \\
\hline
\end{tabular}


Table 1.--Water levels and water-level changes--Continued.

GARFIELD CUUNTY

\begin{tabular}{|c|c|c|c|c|c|c|c|c|c|}
\hline \multirow{2}{*}{$\begin{array}{c}\text { MELL } \\
\text { LOCATION }\end{array}$} & \multirow{2}{*}{ 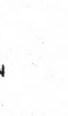 } & \multirow{2}{*}{\multicolumn{3}{|c|}{$\begin{array}{l}\text { PRINCIPAL } \\
\text { AQUIFEN }\end{array}$}} & \multicolumn{2}{|c|}{$\begin{array}{l}\text { WATER LEVEL } \\
1975\end{array}$} & \multirow{2}{*}{$\begin{array}{l}\text { NAIER } \\
\text { LEVEL } \\
\text { CHANGE } \\
1975-76\end{array}$} & \multicolumn{2}{|c|}{$\begin{array}{c}\text { NATER LEVEL } \\
1976\end{array}$} \\
\hline & & & & & DATE & UEPTH & & DATE & DEPTH \\
\hline $021 N=008 N=06$ & UCC & 1 & TERRACE & DEPOSITS & $01-22-75$ & 2.45 & -2.43 & $01-13-70$ & 4.88 \\
\hline $021 N=000 W=10$ & $O C D$ & 1 & IERRACE & DEPOSITS & $01-22-75$ & 1.70 & -2.85 & $01=15-76$ & 4.55 \\
\hline $021 N-008 N-19$ & CBo & 2 & TERRACE & UEPOSITS & $01-22-75$ & 22.22 & 3.68 & $01-15-76$ & 18.54 \\
\hline $023 N-007 N-21$ & $A A D$ & 1 & TERRACE & DEPUSITS & $01-24=75$ & 30.32 & $\star \star \star \star \star \star \star$ & $01-10-70$ & 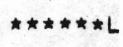 \\
\hline $023 N=007 W-21$ & $\triangle A D$ & 2 & TERRACE & DEPOSITS & $01-24-75$ & 5.96 & -1.96 & $01-16-76$ & 7.92 \\
\hline $023 N-007 N-26$ & $\triangle A O$ & 1 & TERRACE & DEPOSIIS & $01-24-75$ & 29.00 & 1.70 & $01-16-76$ & 27.90 \\
\hline $023 N-007 N=26$ & $B 8 A$ & 3 & TERRACE & OEPUSITS & $01-24-75$ & 30.90 & -0.28 & $01-16-76$ & 39.18 \\
\hline $023 N-007 m-27$ & $\Delta \forall \Delta$ & 1 & TERRACE & DEPOSITS & $01-24-75$ & 51.12 & 2.42 & $01-16-76$ & 48.70 \\
\hline $023 N-007 w-27$ & $60 \mathrm{~A}$ & 3 & TERRACE & DEPDSIIS & $01-24-75$ & 40.58 & -2.23 & $01-16-76$ & 48.81 \\
\hline $023 N-007 N-27$ & $\forall C B$ & 2 & TERRACE & DEPUSITS & $01-24=75$ & 50.50 & 5.44 & $01-16-70$ & 45.06 \\
\hline $023 N-007 N-28$ & $A D A$ & 2 & TERRACE & OEPUSITS & $01-24-75$ & 49.04 & 0.34 & $01-16-76$ & 48.70 \\
\hline $023 N-007 n-28$ & Dod $A$ & 1 & TERRACE & DEPUSITS & $01-24-75$ & 57.04 & 1.02 & $01-16-70$ & 56.02 \\
\hline
\end{tabular}

GARVIN CUUNTY

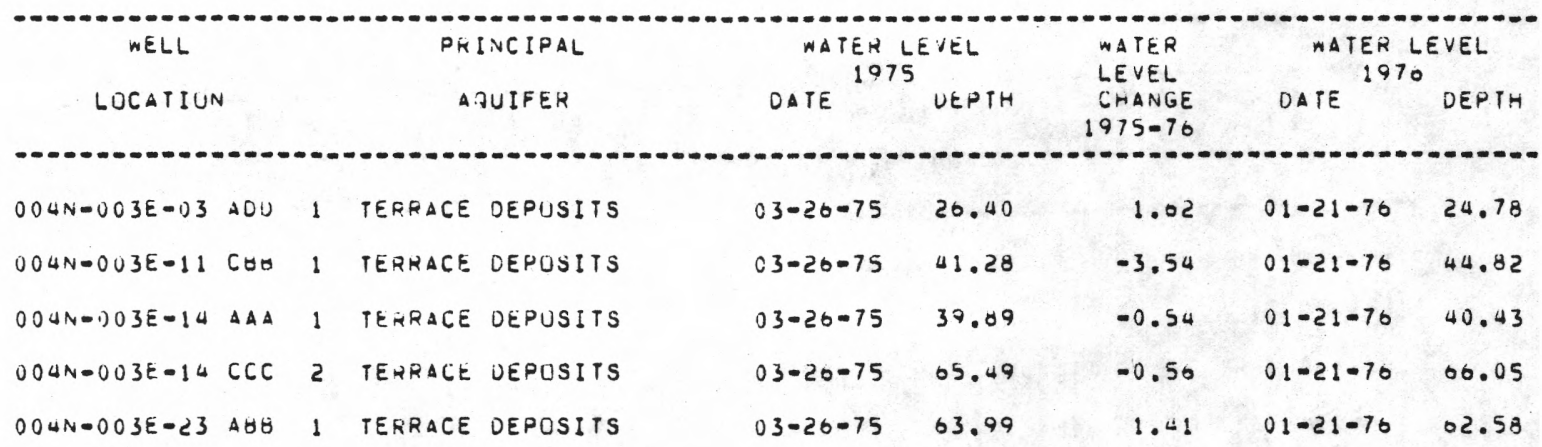


Tauie 1. - inater ieveis aid water-1evel changos--Continued.

GRANT COUNTY

\begin{tabular}{|c|c|c|c|c|c|c|c|}
\hline \multirow{2}{*}{$\begin{array}{l}\text { HELL } \\
\text { LOCATION }\end{array}$} & \multirow{2}{*}{\multicolumn{2}{|c|}{$\begin{array}{l}\text { PRINCIPAL } \\
\text { AWUIFER }\end{array}$}} & \multicolumn{2}{|c|}{$\begin{array}{l}\text { WATER LEVEL } \\
1975\end{array}$} & \multirow{2}{*}{$\begin{array}{l}\text { NATER } \\
\text { LEVEL } \\
\text { CHANGE } \\
1975-76\end{array}$} & \multicolumn{2}{|c|}{$\begin{array}{c}\text { WATER LEVEL } \\
1970\end{array}$} \\
\hline & & & DATE & VEPTH & & OATE & DEPTH \\
\hline $026 N=005 W-31$ AUA & 1 & TERRACE UEPOSITS & $03-06-75$ & 3.05 & -7.25 & $04-07-70$ & 10.30 \\
\hline $026 N=000 N=24000$ & 1 & TERKACE DEPOSITS & $03-06-75$ & 7.50 & -5.33 & $04-07-70$ & 12.03 \\
\hline $026 N=007 N=07 \quad A \theta A$ & 1 & TERRACE DEPOSITS & $03-05-75$ & 3.48 &  & $04-06-76$ & * * \\
\hline $020 N-008 m-27 \quad 0 A A$ & 1 & TERRACE DEPOSITS & $03-05-75$ & 5.05 & -4.37 & $04-00-76$ & 9.42 \\
\hline $027 N-007 W-18$ OAD & 1 & TERRACE DEPOSITS & $03-05-75$ & 5.39 & -4.53 & $04-00-76$ & 9.92 \\
\hline $027 N-008 n=02$ CCC & 1 & TERRACE DEPOSIIS & $03-05-75$ & 2.35 & -4.70 & $04-06-76$ & 7.05 \\
\hline
\end{tabular}

GHEER COUNTY

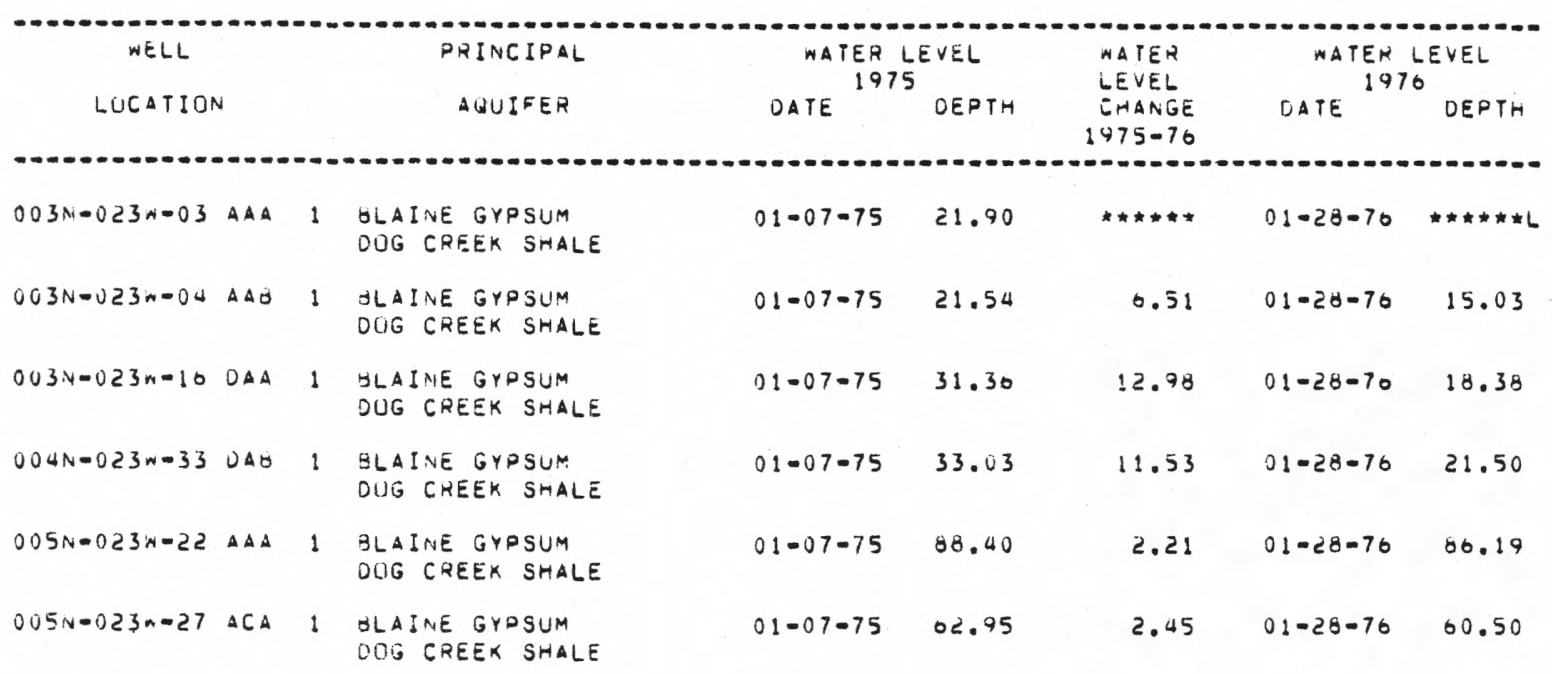


Table 1.-Water levels and water-level changes--Continued.

MARMUN CUUNTY

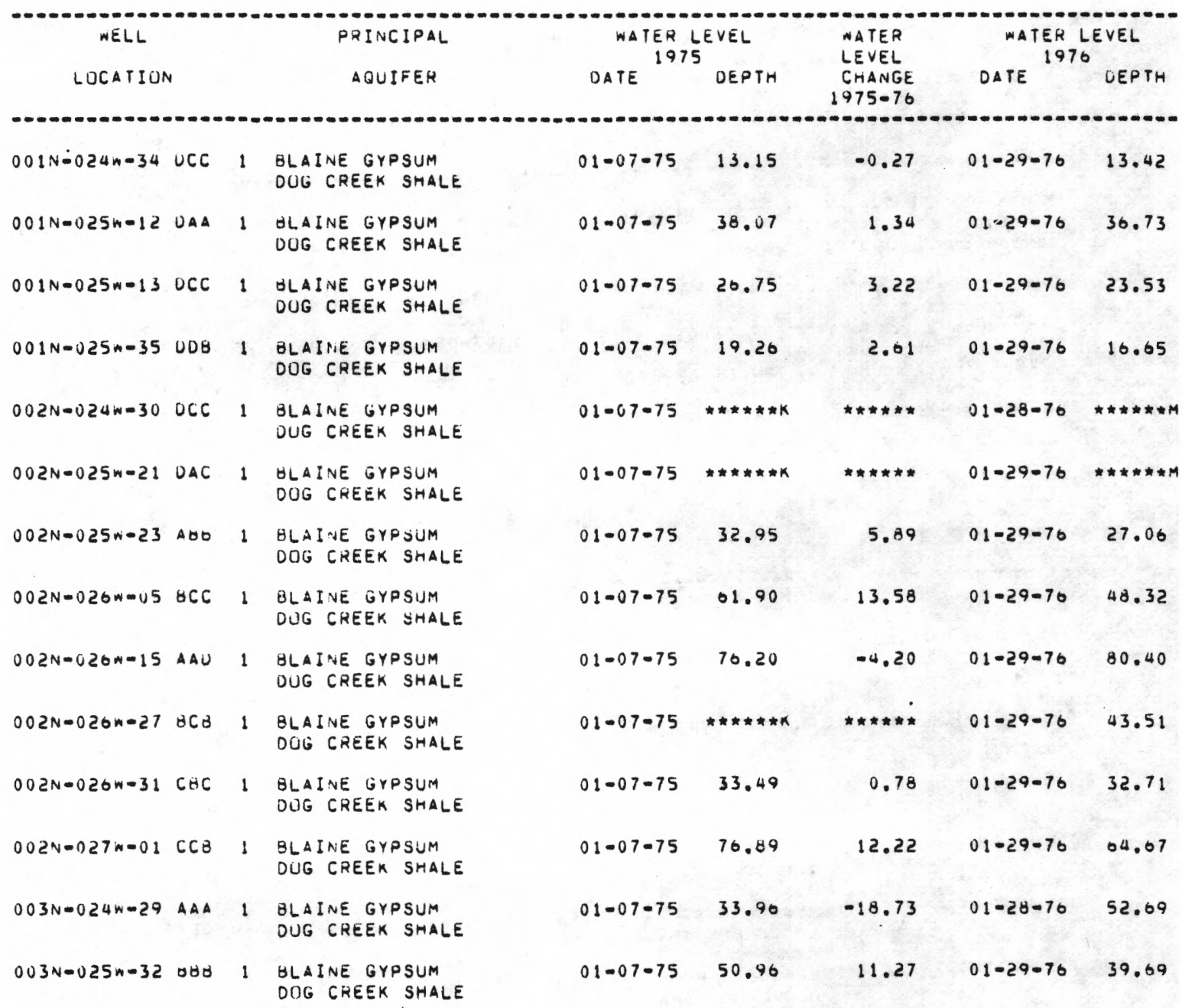


Table 1.-Water levels and water-level changes--Continued. HARPER COUNTY

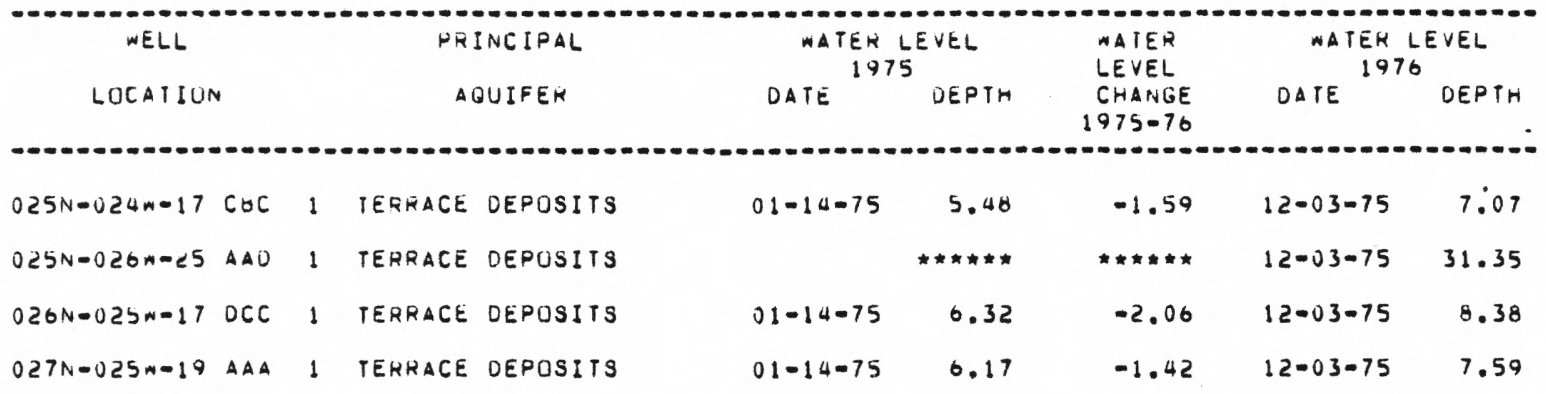

JACKSON COUNTY

\begin{tabular}{|c|c|c|c|c|c|c|c|c|}
\hline \multirow{2}{*}{\multicolumn{2}{|c|}{$\begin{array}{c}\text { WELL } \\
\text { LOCATIUN }\end{array}$}} & \multirow{2}{*}{\multicolumn{2}{|c|}{$\begin{array}{l}\text { PRINCIPAL } \\
\text { AGUIFER }\end{array}$}} & \multicolumn{2}{|c|}{$\begin{array}{l}\text { NATER LEVEL } \\
1975\end{array}$} & \multirow{2}{*}{$\begin{array}{l}\text { AATER } \\
\text { LEVEL } \\
\text { CHANGE } \\
1775=76\end{array}$} & \multicolumn{2}{|c|}{$\begin{array}{l}\text { WATER LEVEL } \\
1976\end{array}$} \\
\hline & & & & OATE & DEPTH & & DATE & OEPTH \\
\hline $0015=024 w-02$ & $\triangle B B$ & 1 & $\begin{array}{l}\text { OLAINE GYPSUM } \\
\text { OUG CREEK SHALE }\end{array}$ & $01-07-75$ & 28.32 & -0.98 & $01-29-76$ & 29.30 \\
\hline $0015-025 w-13$ & $\operatorname{ccc}$ & 1 & $\begin{array}{l}\text { BLAINE GYPSUM } \\
\text { DOG CREEK SHALE }\end{array}$ & $01-07-75$ & 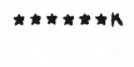 & 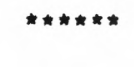 & $01-29-76$ & 72.75 \\
\hline $001 N=023 w-10$ & $\forall \forall \dot{0}$ & 1 & $\begin{array}{l}\text { BLAINE GYPSUM } \\
\text { OOG CREEK SHALE }\end{array}$ & $01-07-75$ & 16.65 & -6.54 & $01-29-76$ & 23.19 \\
\hline $002 N-022 N=08$ & OOA & 1 & $\begin{array}{l}\text { BLAINE GYPSUM } \\
\text { OOG CREEK SHALE }\end{array}$ & $01-07-75$ & 28.59 & 0.86 & $01-28-76$ & 27.73 \\
\hline $0021 n-023 n-12$ & 80 & 1 & $\begin{array}{l}\text { BLAINE GYPSUM } \\
\text { OOG CREEY SHALE }\end{array}$ & $01-07-75$ & 24.48 & -0.61 & $01-28-76$ & 25.09 \\
\hline $002 N-023 N-21$ & $\triangle A A$ & 1 & $\begin{array}{l}\text { BLAINE GYPSUM } \\
\text { OOG CREEK SHALE }\end{array}$ & $01-07-75$ & 10.10 & -3.36 & $01-28-76$ & 21.52 \\
\hline $003 N-122 w-32$ & $\cos$ & 1 & $\begin{array}{l}\text { BLAINE GYPSIJM } \\
\text { DOG CREEK SHALE }\end{array}$ & $01-07-75$ & 34.99 & 0.34 & $01-28-76$ & 34.65 \\
\hline $003 x=023 x-19$ & 680 & 1 & $\begin{array}{l}\text { BLAINE GYPSUM } \\
\text { OOG CREEK SHALE }\end{array}$ & $01-07-75$ & 75.87 & 3.02 & $01-28-70$ & 12.85 \\
\hline $003 N-023 n-27$ & $A A O$ & 1 & $\begin{array}{l}\text { BLAINE GYPSUM } \\
\text { OUG CREEK SHALE }\end{array}$ & $01-0.7-75$ & 35.58 & 4.35 & $01-28-76$ & 31.23 \\
\hline $003 N-023 n-30$ & DAA & 1 & $\begin{array}{l}\text { BLAINE GYPSUM } \\
\text { OOG CREEK SHALE }\end{array}$ & $01-07-75$ & 19.67 & 3.27 & $01-28-70$ & 10.40 \\
\hline
\end{tabular}

KAY COUNTY

PULNCIPAL
LUCATION


Table 1.--Water levels and water-level changes--Continued.

KINGFISHER COUNTY

\begin{tabular}{|c|c|c|c|c|c|c|c|c|c|}
\hline \multirow{2}{*}{$\begin{array}{r}\text { WELL } \\
\text { LOCATIO }\end{array}$} & & \multirow{2}{*}{\multicolumn{3}{|c|}{$\begin{array}{l}\text { PHINCIPAL } \\
\text { AQUIFER }\end{array}$}} & \multicolumn{2}{|c|}{$\begin{array}{l}\text { NATER LEVEL } \\
1975\end{array}$} & \multirow{2}{*}{$\begin{array}{l}\text { NATTR } \\
\text { LEVEL } \\
\text { CHANGE } \\
1975-76\end{array}$} & \multicolumn{2}{|c|}{ MATEK LEVEL } \\
\hline & & & & & DITE & OEPTH & & OATE & DEPTH \\
\hline $017 N-005 N=04$ & 888 & 1 & TERRACE & OEPDSITS & $01-21-75$ & 18.08 & 4.28 & $01-12-76$ & 13.80 \\
\hline $017 N-005 W=00$ & $6 B A$ & 1 & TERRACE & DEPOSITS & $01-21-75$ & 29.75 & 5.39 & $01-12-70$ & 24.36 \\
\hline $017 N-005 N-10$ & $\Delta C C$ & 1 & TERRACE & UEPOSITS & $01-20-75$ & 17.90 & 1.21 & $01-12-76$ & 16.09 \\
\hline $017 N-005 m-13$ & 000 & 1 & TERFACE & DEPOSITS & $01-20-75$ & 20.07 & 3.83 & $01-12-76$ & 24.24 \\
\hline $017 N-005 m-14$ & $\operatorname{coc}$ & 1 & TERRACE & DEPOSITS & $01-20-75$ & 27.11 & 2.15 & $01-12-70$ & 24.96 \\
\hline $017 N-005 n-18$ & $\operatorname{CCC}$ & 1 & TERRACE & DEPOSITS & $01-20-75$ & 24.05 & $=0.67$ & $01-12-76$ & 25.32 \\
\hline $017 N-005 m-20$ & $A A D$ & 1 & TERRACE & DEPOSITS & & 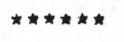 & $\star \star \star \star \star \star \star \star$ & $01-12-70$ & $\star$ \\
\hline $017 N-005 N-27$ & $C C C$ & 1 & TERRACE & DEPOSITS & $01-20-75$ & 4.11 & -1.11 & $01-12-76$ & 5.22 \\
\hline $017 N-000 N-02$ & $\triangle A A$ & 1 & TERRACE & DEPUSITS & $01-21-75$ & 19.54 & 3.70 & $01-12-76$ & 15.84 \\
\hline $017 N=006 N-11$ & ODO & 1 & TERRACE & DEPOSITS & $01-20-75$ & 34.00 & 0.99 & $01-12-76$ & 33.01 \\
\hline $017 N-006 w-12$ & $A A A$ & 1 & TERRACE & UEPOSITS & $01-21-75$ & 37.98 & 3.90 & $01-12-76$ & 34.08 \\
\hline $017 N=000 N-18$ & ECC & 1 & TERRACE & UEPOSITS & $01-21-75$ & 0.18 & -2.04 & $01-12-76$ & 2.22 \\
\hline $017 N=007 N=02$ & D8u & 1 & TERKACE & DEFOSITS & $01-21-75$ & 2.70 & -2.60 & $01-12-76$ & 5.30 \\
\hline $017 N-007 w-11$ & OUA & 1 & TERKACE & DEPOSITS & $01-21-75$ & 9.94 & -1.29 & $01-12-76$ & 11.23 \\
\hline $017 N-007 m-11$ & UCu & 2 & TERRACE & DEPUSITS & $01-21-75$ & 11.05 & -2.35 & $01-12-70$ & 13.40 \\
\hline $017 N-007 N-12$ & DAA & $t$ & TERRACE & DEPIOSITS & $01-21-75$ & 5.52 & -0.46 & $01-12-76$ & 5.98 \\
\hline $010 N=000 N=03$ & COD & 1 & TERRACE & DEPUSITS & $01-21-75$ & 7.25 & 0.70 & $01-13-76$ & 6.55 \\
\hline $018 N=000 N=00$ & 000 & 1 & TERRACE & DEPUSIIS & $01-21=75$ & 17.47 & -0.98 & $01-13-76$ & 18.45 \\
\hline $018 N=000 W=07$ & $A A C$ & 1 & TERRACE & DEPUSITS & $01-21=75$ & 3.06 & -3.49 & $01-13-76$ & 7.15 \\
\hline $013 N-006 N=10$ & 000 & 1 & TERRACE & UEPUSITSS & $01-21-75$ & 2.08 & -1.89 & $01-13-76$ & 4.77 \\
\hline $018 N=000 m-15$ & CAÚ & 1 & TERRACE & UEPUSITS & $01-21-75$ & 1.47 & -1.37 & $01-13-70$ & 2.84 \\
\hline $018 N-006 N-10$ & $c c 0$ & 1 & TERRACE & DEPOSITS & $01-21-75$ & 20.02 & 6.97 & $01-13-76$ & 19.05 \\
\hline $018 N=006 N-17$ & $\triangle A B$ & 1 & TERRACE & DEPOSITS & $01-21=75$ & 30.59 & 5.16 & $01-13-76$ & 31.43 \\
\hline
\end{tabular}


Table 1.-Water levels and water-level changes--Continued.

KINGFISHER COUNTY

\begin{tabular}{|c|c|c|c|c|c|c|c|c|c|}
\hline WELL & & & & INCIPAL & $\begin{array}{r}\text { MATER } \\
191\end{array}$ & EVEL & $\begin{array}{l}\text { NATER } \\
\text { LEVEL }\end{array}$ & $\begin{array}{r}\text { WATER } \\
197\end{array}$ & EVEL \\
\hline LOCATIUU & & & & QUIFER & DATE & DEPTH & $\begin{array}{l}\text { CHANGE } \\
1975-76\end{array}$ & DATE & DEPTH \\
\hline $018 N=006 W-17$ & 080 & 2 & TERRACE & DEPOSITS & $01-21-75$ & 9.90 & -1.07 & $01-13-76$ & 10.97 \\
\hline $018 N-000 W=20$ & $C C A$ & 1 & TERRACE & UEPUSITS & $01-21-75$ & 12.43 & 4.43 & $01-13-76$ & 8.00 \\
\hline $018 N-000 N-22$ & $\cos$ & 1 & TERRACE & UEPUSITS & $01-21-75$ & 38.33 & 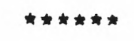 & $01-13-76$ & 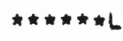 \\
\hline $018 N=000 N-31$ & $B A A$ & $!$ & TERRACE & DEPISITS & $01-21-75$ & 13.05 & 1.37 & $01-13-76$ & 11.08 \\
\hline $018 N-000 W-31$ & טuo & 2 & TERRACE & DEPUSITS & $01-21-75$ & 2.74 & -0.18 & $01-12-76$ & 2.92 \\
\hline $018 N-000 w-32$ & $B A A$ & 1 & TERRACE & DEPOSITS & $01-21-75$ & 9.32 & 0.47 & $01-13-76$ & 8.85 \\
\hline $018 N-000 m-35$ & 880 & 1 & TERRACE & DEPUSITS & $01-21-75$ & 10.37 & 3.43 & $01-13-70$ & 12.94 \\
\hline $016 N=007 N=21$ & OCD & 1 & TERRACE & DEPOSITS & $01-21-75$ & 22.49 & 1.57 & $01=13-76$ & 20.92 \\
\hline $018 N-\cup 08 W=10$ & $\triangle A O$ & 1 & TERRACE & UEPUSITS & $01-22-75$ & 11.09 & -0.60 & $01-13-70$ & 11.09 \\
\hline $018 N-008 W=13$ & 888 & 1 & TERRACE & DEPIJSITS & $01-22-75$ & 0.69 & -1.21 & $01-13-70$ & 1.90 \\
\hline $019 N-007 m=08$ & $\triangle O A$ & 1 & TERFACE & DEPOSITS & $01-22-75$ & 10.73 & -2.55 & $01-13-70$ & 13.28 \\
\hline $019 N-007 W=22$ & $A \triangle B$ & 1 & TERRACE & DEPOSITS & $01-22-75$ & 13.18 & -2.91 & $01-13-70$ & 16.09 \\
\hline $019 N-007 m-22$ & $B A A$ & 2 & TERRACE & DEPUSITS & $01-22-75$ & 4.20 & -3.34 & $01-13-76$ & 7.54 \\
\hline $019 N-007 W=30$ & OCC & 2 & TERRACE & DEPOSITS & $01-22-75$ & $\star \star *$ & $m * * * * *$ & $01-13-70$ & 29.95 \\
\hline $019 N=008 W=03$ & $\triangle A A$ & 1 & TERRACE & DEPOSITS & $01-22-75$ & 1.90 & -1.14 & $01-13-70$ & 3.04 \\
\hline $019 N-008 m-17$ & $O C O$ & 1 & TERRACE & DEPUSITS & $01-22-75$ & 7.72 & 2.83 & $01-13-76$ & 4.89 \\
\hline $019 N-000 N-27$ & $D A C$ & 1 & TERRACK & DEPOSITS & $01-22-75$ & 7.40 & 2.77 & $01-13-76$ & 4.63 \\
\hline $019 N-009 N-10$ & COO & 1 & TERIACE & DEPOSITS & $01-22-75$ & 3.29 & -0.09 & $01-14=70$ & 3.38 \\
\hline $019 N=009 W=24$ & $A A D$ & 1 & TERRACE & DEPOSITS & $01-22-75$ & 0.87 & 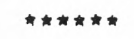 & & 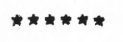 \\
\hline
\end{tabular}


Table 1.-Water levels and water-level changes--Continued.

$$
\text { KIUNA COUNTY }
$$

\begin{tabular}{|c|c|c|c|c|c|c|c|c|c|}
\hline \multirow{2}{*}{$\begin{array}{c}\text { WELL } \\
\text { LOCATION }\end{array}$} & \multirow{2}{*}{ 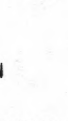 } & \multirow{2}{*}{\multicolumn{3}{|c|}{$\begin{array}{l}\text { PRINCIFAL } \\
\text { AQUIFER }\end{array}$}} & \multicolumn{2}{|c|}{$\begin{array}{l}\text { WATER LEVEL } \\
1975\end{array}$} & \multirow{2}{*}{$\begin{array}{l}\text { WATER } \\
\text { LEVEL } \\
\text { CHANGE } \\
1975=76\end{array}$} & \multicolumn{2}{|c|}{$\begin{array}{c}\text { WATEF LEVEL } \\
1970\end{array}$} \\
\hline & & & & & UATE & DEPTH & & DATE & DEFTH \\
\hline $002 N-017 n=10$ & DCL & 1 & TERRACE & DEPOSITS & & $\hbar \star \star \star \star \star \star \star *$ & $\star \star \star * \star \star \star \star$ & $01-28=76$ & 25.45 \\
\hline $002 N=017 W=17$ & $A D D$ & 1 & TERRACE & UEPUSITS & & $\star \star \star \star \star \star \star \star$ & $\star \star \star \star \star \star \star * \hbar$ & $01-28=76$ & 15.55 \\
\hline $003 N=017 N=28$ & CAU & 1 & TERRACE & DEPOSIIS & & $\hbar * \star \star \star \star \star *$ & 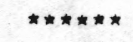 & $01-28-70$ & 20.69 \\
\hline $006 N=020 W=20$ & $A D A$ & 2 & IERRACE & DEPUSITS & $01-08-75$ & 33.45 & 3.21 & $02-09-70$ & 30.24 \\
\hline $006 N-020 W-20$ & DAA & 1 & TERRACE & DEPOSITS & $01-08-75$ & 31.78 & 4.25 & $02-09-76$ & 27.53 \\
\hline $006 N-020 K=21$ & $C C A$ & 1 & TERRACE & UEPOSITS & $01-08-75$ & 38.14 & 4.67 & $02-09-76$ & 33.47 \\
\hline $000 N-020 w-21$ & $D C C$ & 2 & TERRACE & DEPOSITS & $01-08-75$ & 35.40 & 5.62 & $02=09-76$ & 29.78 \\
\hline $000 N-020 W=23$ & $\operatorname{coc}$ & 1 & TERRACE & DEPOSITS & $01=08-75$ & 15.83 & 2.61 & $02=09-76$ & 13.22 \\
\hline $000 N-020 w-27$ & $\operatorname{ccc}$ & 1 & TEKRACE & DEPUISITS & $01-00-75$ & 26.00 & 2.05 & $02-09-70$ & 24.03 \\
\hline $000 N=020 N=35$ & DAA & 1 & TERRACE & UEROSITS & $01-08-75$ & 7.50 & 1.33 & $02=09-76$ & 0.17 \\
\hline $007 N=018 w=32$ & $\forall C d$ & 1 & TERRACE & DEPUSITS & $01-08=75$ & 19.43 & 1.03 & $02-09-76$ & 18.40 \\
\hline $007 N-015 n-14$ & $B C C$ & 1 & TERRACE & DEPUSITS & $01=09-75$ & 19.20 & 1.28 & $02=09-76$ & 17.92 \\
\hline
\end{tabular}

LUGAN COUNTY

\begin{tabular}{|c|c|c|c|c|c|c|c|c|c|}
\hline \multirow{2}{*}{\multicolumn{2}{|c|}{ LOCATIUN }} & \multirow{2}{*}{\multicolumn{3}{|c|}{$\begin{array}{l}\text { PRINCIPAL } \\
\text { AQUIFER }\end{array}$}} & \multicolumn{2}{|c|}{$\begin{array}{l}\text { WATER LEVEL } \\
1975\end{array}$} & \multirow{2}{*}{$\begin{array}{l}\text { NATER } \\
\text { LEVEL } \\
\text { CHANGE } \\
1975-76\end{array}$} & \multicolumn{2}{|c|}{$\begin{array}{l}\text { WATEK LEVEL } \\
1976\end{array}$} \\
\hline & & & & & DATE & DEPTH & & DATE & UEEPTH \\
\hline $016 N=004 n=03$ & AOO & 1 & TERRACE & DEPOSITS & $01-20-75$ & 23.50 & -1.15 & $01-12-76$ & 24.65 \\
\hline $017 N=004 w-15$ & $\cos$ & 1 & TERTACE & UEPUSITS & $01-20-75$ & 12.53 & 1.41 & $v 1-12-70$ & 11.12 \\
\hline $017 N-004 W-19$ & AOU & 1 & TERRACE & DEPUSITS & $01-20-75$ & 22.42 & 4.53 & $01-12-76$ & 17.89 \\
\hline $017 N=004 N-27$ & $\mathrm{CBB}$ & 1 & PERRACE & DEPUSITS & $01=20-75$ & 4.85 & 1.20 & $01=: 2=70$ & 3.65 \\
\hline $017 N=004 N=27$ & $\operatorname{COC}$ & 2 & TERRACE & DEPUSITS & $01-20-75$ & 0.03 & 1.25 & $01-12-76$ & 5.58 \\
\hline $017 N-004 N-31$ & $\mathrm{CCC}$ & 1 & TERRACE & DEPOSITS & $01-20-75$ & 20.12 & 1.44 & $01-12-70$ & 18.68 \\
\hline $017 N=004 N-34$ & $A B O$ & $!$ & TERKACE & DEPUSITS & $01-20-75$ & 29.00 & 1.23 & $01-12-70$ & 27.77 \\
\hline $017 N=004 N-34$ & cco & 2 & TERRACE & DEPOSITS & $01-20-75$ & 49.33 & 1.96 & $01-12-70$ & 47.37 \\
\hline $017 N-004 m-34$ & $\operatorname{COC}$ & 3 & TERRACE & OEPOSITS & $01-20-75$ & 25.55 & 0.79 & $01-12-76$ & 24.76 \\
\hline $017 N=004 m=34$ & $D C C$ & 4 & TERRACE & DEPOSITS & $01=20-75$ & 4.05 & -1.11 & $01-12-76$ & 5.96 \\
\hline
\end{tabular}


Table 1.--Water levels and water-level changes--Continued.

MAJOR COUNTY

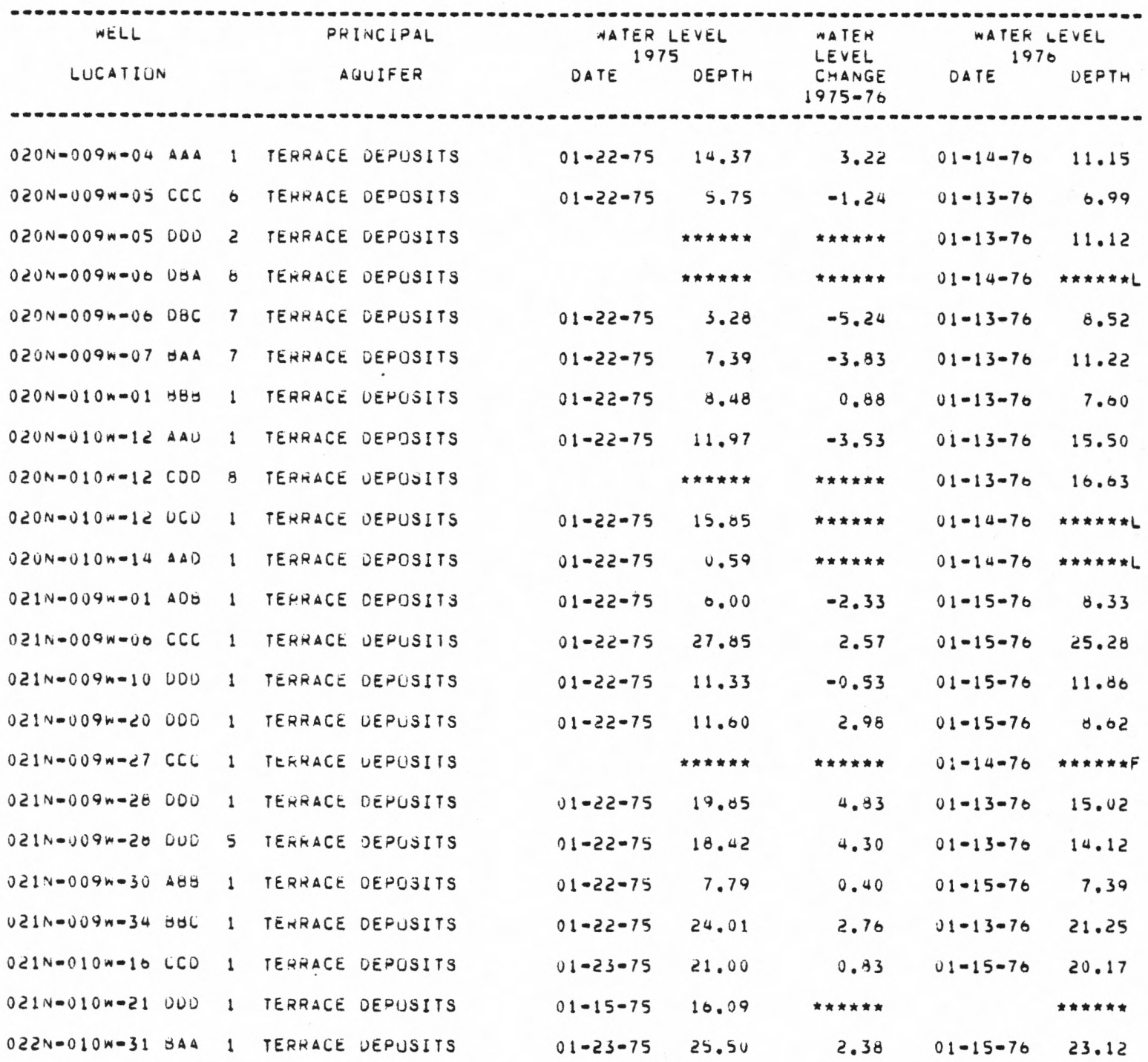


Table 1.--Water levels and water-level changes-Continued.

$$
\text { MAJUR CUUNTY }
$$

WELL
LUCAIIUN
ARUIFER

ROGER MILLS CUUNTY

\begin{tabular}{|c|c|c|c|c|c|c|c|}
\hline \multirow{2}{*}{$\begin{array}{l}\text { WELL } \\
\text { LOCATIUN }\end{array}$} & \multirow{2}{*}{\multicolumn{2}{|c|}{$\begin{array}{l}\text { PRINCIPAL } \\
\text { AQUIFER }\end{array}$}} & \multicolumn{2}{|c|}{$\begin{array}{c}\text { WATER LEVEL } \\
1975\end{array}$} & \multirow{2}{*}{$\begin{array}{l}\text { NATER } \\
\text { LEVEL } \\
\text { CHANGE } \\
1975-76\end{array}$} & \multicolumn{2}{|c|}{$\begin{array}{l}\text { WATER LEVEL } \\
1976\end{array}$} \\
\hline & & & DATE & DEPTH & & DATE & DEPTH \\
\hline $013 N=023 W=08 \quad A 00$ & 1 & ALLUVIUM & & 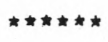 & $\| \hbar \star \star \star \star \star$ & $02-10-76$ & 10.10 \\
\hline $014 N-023 W=10 \quad \Delta \Delta G$ & 1 & ALLUVIUM & & 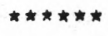 & 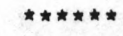 & $02-10=76$ & 14.42 \\
\hline $014 N-023 w-32$ 80U & 1 & ALLUVIUM & & 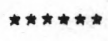 & 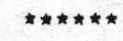 & $02-10-76$ & $35 . .43$ \\
\hline $015 N=024 N=30 \quad C A C$ & 1 & ALLUVIUM & & $\hbar \star \star \star \star \star \star \star$ & 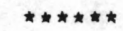 & $02-10-76$ & 7.36 \\
\hline
\end{tabular}


Table 1.-Water levels and water-level changes--Continued.

TEXAS COUNTY

\begin{tabular}{|c|c|c|c|c|c|c|c|c|c|}
\hline NELL & & & PRIN & $N C I P A L$ & $\begin{array}{r}\text { WATER } \\
197\end{array}$ & LEVEL & $\begin{array}{l}\text { NATER } \\
\text { LEVEL }\end{array}$ & $\begin{aligned} \text { WATER } \\
197\end{aligned}$ & LEVEL \\
\hline LOCATION & & & $\triangle Q U$ & UIFER & DATE & DEPTH & CHANGE & DATE & DEPTH \\
\hline $001 S-010 E-02$ & 800 & 1 & OGALLALA & FURMATION & $01-21-75$ & 106.77 & -2.77 & $01-13-7 b$ & 109.54 \\
\hline $001 S-011 E-03$ & $\triangle A A$ & 1 & OGALLALA & FORMATION & $01-21-75$ & 125.57 & -2.91 & $01-14-76$ & 128.48 \\
\hline $001 S=016 E=06$ & $\triangle A A$ & 1 & OGALLALA & FORMATIUN & $01-22-75$ & 243.47 & -2.72 & $01-14-70$ & 240.19 \\
\hline $001 N=010 E=09$ & $\operatorname{coc}$ & 1 & OGALLALA & FORMATION & $01-21-75$ & 202.15 & -6.05 & $01-13-76$ & 208.20 \\
\hline $001 N-010 E-12$ & $D C D$ & 1 & OGALLALA & FORMATIUN & $01-21-75$ & 158.03 & -2.80 & $01-13-70$ & 160.83 \\
\hline $001 N-010 E-15$ & $\triangle C \forall$ & 1 & OGALLALA & FORMATION & $01-21-75$ & 189.05 & -4.23 & $01-13-76$ & 193.28 \\
\hline $01 N-010 E=32$ & CBA & 1 & OGALLALA & FORMATION & $01-21-75$ & 257.73 & -2.25 & $0:-13-70$ & 259.98 \\
\hline $001 N-011 \varepsilon-05$ & $\operatorname{CBO}$ & 1 & OGALLALA & FORMATION & $01-21-75$ & 101.40 & -3.56 & $01-13-76$ & 104.96 \\
\hline $001 N-011 E-22$ & $D D C$ & 1 & OGALLALA & FORMATIUN & $01-21-75$ & 120.278 & 1.808 & $01-13-70$ & 118.47 \\
\hline $001 N-011 E-20$ & 650 & 1 & DGALLALA & FURMATION & $01-21-75$ & 122.89 & -1.99 & $01-13=76$ & 124.88 \\
\hline $001 N=012 E-00$ & DCD & 1 & ALLUVIUM & & $01-21-75$ & 84.08 & -0.82 & $01-13=76$ & 84.90 \\
\hline $01 N-012 E-11$ & 086 & 1 & OGALLALA & FORMATION & $01-21-75$ & 252.32 & -2.50 & $01-13-76$ & 254.88 \\
\hline $001 N-012 E-19$ & 600 & 1 & UGALLALA & FORMATION & $01-21-75$ & $\star \star \star \star \star \star \star A$ & 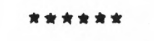 & $01-13-76$ & 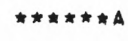 \\
\hline $001 N-012 E-35$ & bu & 1 & OGALLALA & FOHMATION & $01-21-75$ & 196.39 & -1.20 & $01-13-76$ & 197.59 \\
\hline $001 N-V 13 E-01$ & $B C O$ & 2 & DGALLALA & FURMATION & $01-21-75$ & 175.51 & 1.52 & $01-13-76$ & 173.99 \\
\hline $001 N-013 t-10$ & 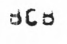 & 1 & OGALLALA & FUKMATIUN & $01-21-75$ & 174.18 & -3.35 & $01-14-70$ & 177.53 \\
\hline $001 N-013 E-26$ & DUA & 1 & UGALLALA & FORMATION & $01-21-75$ & 100.20 & -2.42 & $01-13-70$ & 168.00 \\
\hline $001 N-014 E=10$ & 800 & 1 & UGALLALA & FURMATIUN & $01-22-75$ & 81.71 & -1.28 & $01-13-76$ & 82.99 \\
\hline $001 N-014 E-31$ & $68 \theta$ & 1 & OGALLALA & FURMATIUN & $01-21=75$ & 217.05 & -2.45 & $01-13-76$ & 219.50 \\
\hline $001 N-015 E-10$ & 888 & 1 & OGALLALA & FORMAIIDN & $01-22-75$ & 177.68 & -1.29 & $01-13-76$ & 178.97 \\
\hline $001 N=015 E-19$ & $D A A$ & 1 & UGALLALA & FORMATION & $01-22-75$ & 177.68 & 0.08 & $01-13-70$ & 177.60 \\
\hline $001 N=015 E-27$ & $\operatorname{CCB}$ & 1 & OGALLALA & FORMATIUN & $01-22-75$ & 82.21 & -1.41 & $01-14-76$ & 83.62 \\
\hline $001 N-015 E-32$ & $\cos$ & 1 & DGALLALA & FORMAIIUN & $01-22-75$ & 207.58 & -3.61 & $01-14-76$ & 211.19 \\
\hline
\end{tabular}


Table 1.-Water levels and water-level changes-Continued. TEXAS COUNTY

\begin{tabular}{|c|c|c|c|c|c|c|c|c|c|}
\hline WELLL & & & PHIN & VCIPAL & $\begin{array}{r}\text { NATER } \\
197\end{array}$ & LEVEL & $\begin{array}{l}\text { WATER } \\
\text { LEVEL }\end{array}$ & $\begin{array}{r}\text { WATER } \\
197\end{array}$ & LEVEL \\
\hline LOCATIOA & & & $A Q U$ & JIFER & DATE & UEPTH & $\begin{array}{l}\text { CHANGE } \\
1975-76\end{array}$ & UATE & DENTH \\
\hline $001 N=010 E-23$ & $A B C$ & 1 & UGALLALA & FURMATIUN & $01-22-75$ & 210.00 & -2.90 & $01-14=76$ & 219.50 \\
\hline $001 N-017 E-01$ & Cec & 1 & OGALLALA & FURMAIION & $01-22-75$ & 128.72 & -2.15 & $01-14-76$ & 130.87 \\
\hline $001 N-017 E-10$ & CCC & 1 & UGALLALA & FURMATIUN & $01-22-75$ & 148.06 & 2.30 & $01-14-76$ & 145.76 \\
\hline $001 N=017 E-19$ & $\operatorname{CCC}$ & 1 & JGALLALA & FORMATIUN & $01-22-75$ & 182.34 & -0.32 & $01-14-76$ & 182.06 \\
\hline $001 N-017 E-30$ & 000 & 1 & OGALLALA & FURMATIUN & $01-22-75$ & 169.47 & -1.12 & $01-14-76$ & 170.59 \\
\hline $001 N-018 E-13$ & $\operatorname{coc}$ & 1 & OGALLALA & FUKMATIUN & $01-22-75$ & 12.52 & -0.91 & $01-14-76$ & 13.43 \\
\hline $001 N=018 E-34$ & $B B A$ & 1 & OGALLALA & FORMATION & $01-22-75$ & 73.91 & 0.30 & $01-14=76$ & 73.61 \\
\hline $001 N-019 t-30$ & 000 & 1 & OGALLALA & FORMATION & $01-22-75$ & 36.98 & -4.47 & $01-14-70$ & 41.45 \\
\hline $001 N-019 E-33$ & $\Delta \Delta A$ & 1 & OGALLALA & FURMATION & $01-22-75$ & 113.06 & $\star * * * * * *$ & $01-14=70$ & $\star \star \star \star \star \star \star \star * H$ \\
\hline $002 N-010 E-20$ & 800 & 1 & UGALLALA & FORMATION & $01-21-75$ & 212.04 & $* * *$ & $01-13=76$ & $* \star \Delta$ \\
\hline $002 N=010 E-2 Z$ & 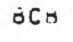 & 1 & DGALLALA & FORMATIUN & $01-2 !-75$ & 177.08 & -2.84 & $01-13=76$ & 179.92 \\
\hline $002 N-011 E-34$ & $C C \theta$ & 1 & OGALLALA & FORMATIUN & $01-21-75$ & 101.03 & -3.52 & $0:-13=76$ & 104.55 \\
\hline $002 N-012 t-25$ & UBO & 1 & UGALLALA & FURMATIUN & $01-21-75$ & 25 & 1.24 & $01-13-70$ & 256.92 \\
\hline $002 N=012 E-33$ & $\triangle A D$ & 1 & DGALLALA & FORMATIUN & $01-21-75$ & $\star \star \star \star \star \star \star A$ & $\star \star \star \star \star \star \star \star *$ & $01-13-76$ & $\star \star \star \star \star \star \star \star \Delta$ \\
\hline $002 N=013 E-13$ & $C A A$ & 1 & JGALLALA & FORMATIUN & $01-21-75$ & 178.89 & -3.42 & $01-14=76$ & 182.31 \\
\hline $002 N-013 E-16$ & 848 & 1 & OGALLALA & FOKKMATION & $01-21-75$ & 222.18 & $\times 2 \times$ & $01-13-70$ & $x-$ \\
\hline $002 N=013 E-32$ & $\mathrm{CCC}$ & 1 & OGALLALA & FURMATION & $01-21-75$ & $\# \star \star \star \star \star \star A$ & 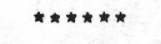 & $01-13-76$ & 214.02 \\
\hline $002 N-014 E-11$ & $\operatorname{cy} A$ & 1 & OGALLALA & FURIAATIUN & $01-21-75$ & 146.91 & -2.46 & $01-13-76$ & 149.37 \\
\hline $002 N-014 E-23$ & $\cos$ & 2 & UGALLALA & FORMATION & $01-21-75$ & 150.65 & -2.18 & $01-14-76$ & 152.83 \\
\hline $002 N=015 E-09$ & $B C \theta$ & 1 & OGALLALA & FURMATIUN & $01-21-75$ & $259.30 c$ & $-7.38 \mathrm{C}$ & $01-13-76$ & 260.68 \\
\hline $002 N-015 E-15$ & OAA & 1 & OGALLALA & FORMATION & $01-23-75$ & 249.400 & -16.256 & $01-14-70$ & 205.050 \\
\hline $002 N=016 t=04$ & OBA & 1 & DGALLALA & FURMATION & $01-23-75$ & 200.58 & -3.76 & $01-15-70$ & 217.34 \\
\hline $0 \cup 2 N=016 E-07$ & $a b \theta$ & 1 & OGALLALA & FORMATIUN & $01-23-75$ & 271.00 & -6.89 & $01-15-70$ & 277.89 \\
\hline
\end{tabular}


Table 1.--Water levels and water-level changes--Continued.

TEXAS COUNTY

\begin{tabular}{|c|c|c|c|c|c|c|c|c|c|}
\hline NELL & & & $P R$ & N & $\begin{array}{r}\text { WATER } \\
197\end{array}$ & - & $\begin{array}{l}\text { NATER } \\
\text { LEVEL }\end{array}$ & $\begin{array}{r}\text { WATER } \\
197\end{array}$ & LEVE \\
\hline LOCATION & & & $\Delta Q$ & UIFER & DATE & UEPTH & $\begin{array}{r}\text { CHANGE } \\
1975-76\end{array}$ & DATE & DEPTH \\
\hline $002 N=010 E-08$ & $\triangle A A$ & 1 & UGALLALA & FORMATION & $01-23-75$ & 220.450 & 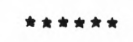 & $01-15-76$ & $\star \star \star \star \star \star \star \star A$ \\
\hline $002 N=010 E-14$ & $C \forall B$ & 1 & OGALLALA & FORMATIUN & $01-23-75$ & 190.14 & -2.40 & $01-15-70$ & 198.54 \\
\hline $002 N=017 E-07$ & $6 C C$ & 1 & OGALLALA & FORMATION & $01-23-75$ & 190.17 & -4.82 & $01-15-76$ & 194.99 \\
\hline $002 N-017 E-11$ & 800 & 1 & ALLUVIUM & & $01-23-75$ & 10.77 & -1.10 & $01-14=76$ & 11.87 \\
\hline $002 N-017 E-15$ & $A B O$ & 1 & ALLUVIUM & & $01-23-75$ & 8.44 & $\star \star \star \star \star \star \star \star *$ & $01-14-76$ & $\star \star \star \star \star \star \star \star F F$ \\
\hline $002 N-017 E-35$ & 608 & 1 & CGALLALA & FORMATION & & 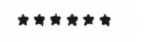 & $\star \star \star \star \star \star \star$ & $01-14-70$ & 175.19 \\
\hline $002 N=018 E=03$ & $\triangle D C$ & 1 & DGALLALA & FURMATIUN & $01-22-75$ & 3.01 & -0.33 & $01-14-76$ & 3.34 \\
\hline $002 N=018 E-08$ & $8 \Delta 0$ & 1 & DGALLALA & FURMATION & $01-23-75$ & 95.05 & 0.35 & $01-14-76$ & 94.70 \\
\hline $00 Z N=010 E-11$ & 000 & 1 & DGALLALA & FORMATIUN & $01-22-75$ & 77.80 & -1.37 & $01-14-70$ & 79.17 \\
\hline $002 N-010 E-14$ & OCD & 1 & OGALLALA & FURMATIUN & $01-22-75$ & 162.09 & 0.16 & $01-14-76$ & 162.73 \\
\hline $002 N=018 E-24$ & $\triangle C C$ & 1 & $U G A L L A L A$ & FURMATIUN & $01-22-75$ & 142.60 & -1.87 & $01-14=76$ & 144.47 \\
\hline $002 N-018 E-27$ & $\forall C C$ & 1 & UGALLALA & FORMATION & $01-22-75$ & 144.38 & -3.08 & $01-14-76$ & 148.06 \\
\hline $002 N-01 Z E-3 U$ & C8O & 1 & UGALLALA & FORMATION & & $\star \star \star \star \star \star \star \star *$ & 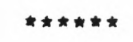 & $01-14-76$ & 181.29 \\
\hline $002 y-010 \varepsilon-34$ & $\cos$ & 1 & OGALLALA & FURMATION & $01-22-75$ & 112.25 & -0.23 & $01-14-76$ & 112.48 \\
\hline $002 N=019 E=07$ & CAA & 1 & NGALLALA & FURMATION & $01-22-75$ & 52.25 & -5.24 & $01-14-76$ & 57.49 \\
\hline $003 N-010 E-11$ & dod & 2 & OGALLALA & FURMATIUN & $01-21-75$ & 121.58 & $-1.18^{\circ}$ & $01-13-76$ & 122.76 \\
\hline $003 N-010 E-19$ & $\operatorname{COC}$ & 1 & OGALLALA & FURMATION & $01-21-75$ & 220.22 & 0.29 & $01-13=70$ & 219.93 \\
\hline $003 N=010 E=24$ & $00 \theta$ & 1 & CGALLALA & FOKMATION & $01-21-75$ & 181.18 & -1.37 & $01-13-76$ & 182.55 \\
\hline $003 N=011 E-10$ & $C B C$ & 1 & OGALLALA & FURMATIUN & $01-21-75$ & 155.05 & -2.21 & $01-13-76$ & 157.20 \\
\hline $003 N=013 E-02$ & COU & 1 & UGALLALA & FORMATIUN & $01-21-75$ & 62.14 & 0.17 & $01-13-70$ & 61.97 \\
\hline $003 N-015 E-20$ & $\cos$ & 1 & IJGALLALA & FIJRMATION & $01-23-75$ & 201.73 & -4.20 & $01-15-70$ & 205.93 \\
\hline $003 N=010 E=03$ & $\mathrm{OBC}$ & 1 & OGALLALA & FURMATIUN & $01-22-75$ & 82.59 & 0.16 & $01-15-70$ & 82.43 \\
\hline $003 N=016 E-10$ & $\Delta \in \sigma$ & 1 & ALLUVIUM & & $01-22-75$ & 15.79 & -0.51 & $01-14-76$ & 16.30 \\
\hline
\end{tabular}


Table 1.--Water levels and water-level changes--Continued.

TEXAS CUUNTY

\begin{tabular}{|c|c|c|c|c|c|c|c|c|c|}
\hline \multirow{2}{*}{$\begin{array}{c}\text { WELL } \\
\text { LUCATION }\end{array}$} & & \multirow{2}{*}{\multicolumn{3}{|c|}{$\begin{array}{l}\text { PRINCIPAL } \\
\text { AIUIFER }\end{array}$}} & \multicolumn{2}{|c|}{$\begin{array}{l}\text { WATER LEVEL } \\
1975\end{array}$} & \multirow{2}{*}{$\begin{array}{l}\text { WATER } \\
\text { LEVEL } \\
\text { CHANGE } \\
1975-76\end{array}$} & \multicolumn{2}{|c|}{$\begin{array}{c}\text { WATEK LEVEL } \\
1976\end{array}$} \\
\hline & & & & & DATE & DEPTH & & DATE & DEPTH \\
\hline $003 N-016 t-23$ & $\Delta \Delta Q$ & 1 & OGALLALA & FURMATIUN & $01-23-75$ & 114.58 & 0.49 & $01-14-76$ & 114.09 \\
\hline $003 N-016 E-28$ & $\triangle A U$ & 1 & OGALLALA & FURMATION & $01-23-75$ & 120.58 & 0.55 & $01-14-76$ & 120.03 \\
\hline $003 N=017 E=00$ & $B A D$ & 1 & OGALLALA & FURMATION & $01-22-75$ & 89.33 & -0.33 & $01-14-76$ & 89.66 \\
\hline $003 N=017 E-13$ & $\cos$ & 1 & OGALLALA & FUKMAIIUN & $01-22-75$ & 87.09 & 1.71 & $01-15-76$ & 85.38 \\
\hline $003 N-017 E-36$ & 800 & 1 & OGALLALA & FORMATION & $01-23-75$ & 72.29 & -3.81 & $01-15-76$ & 96.10 \\
\hline $003 N-016 E=07$ & $A \Delta t$ & 1 & JGALLALA & FURMATION & $01-22-75$ & 126.71 & 1.95 & $01-15-76$ & 124.76 \\
\hline $003 N-018 E-11$ & $\triangle A B$ & 1 & OGALLALA & FURMATIUN & $01-22-75$ & 80.32 & -0.44 & $01-15-76$ & 80.76 \\
\hline $003 N-019 E=02$ & $B \forall C$ & 1 & UGALLALA & FURMATIUN & $01-22-75$ & 94.83 & -1.02 & $01-15-76$ & 95.85 \\
\hline $0 O U N=010 E-18$ & CCS & 1 & DOCKIJM G & roup & $01-21-75$ & 212.00 & 1.50 & $01-13-76$ & 210.50 \\
\hline $004 N=010 E-31$ & $A 00$ & 1 & DOJCXUM G & RUUP & $01-21-75$ & $\star \star \star \star \star \star \star \star \star x$ & 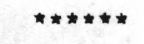 & $01-16-70$ & 227.43 \\
\hline $004 N=011 E=04$ & 080 & 1 & DOCKUIM G & ROUP & $01-21-75$ & 170.99 & -5.77 & $01-16-76$ & 176.76 \\
\hline $004 N-011 E-10$ & 080 & 1 & DUCKUM G & RUIUP & $01-21-75$ & 150.50 & -1.09 & $01=13-76$ & .59 \\
\hline $004 N-012 E-03$ & 000 & 1 & OGALLALA & FORMATIGIN & $01-21-15$ & 136.10 & -3.70 & $01-16-76$ & 139.80 \\
\hline $004 N-013 E-27$ & $\triangle C O$ & 1 & OGALLALA & FURMATIUN & $01-21-75$ & 114.85 & -0.88 & $01-16-70$ & 115.73 \\
\hline $004 N-014 t=03$ & 008 & 1 & UGALLALA & FURMATIUN & $01-22-75$ & 201.50 & -1.30 & $01-14-76$ & 20 \\
\hline $004 N=014 E-0 S$ & $\Delta \forall C$ & 1 & UGALLALA & FURMATION & $01-22-75$ & 104.00 & -5.40 & $01-14-70$ & 169.40 \\
\hline $004 N-014 E-14$ & $A D C$ & 1 & OGALLALA & FOHMATIUN & $01-22-75$ & $\star \star \star \star \star \star \star A$ & $\star \star \star \star \star \star \star \star$ & $01-12-70$ & 183.15 \\
\hline $004 N=015 E=09$ & $A \cup D$ & 1 & UGALLALA & FORMATION & $01-22-75$ & 173.08 & -3.92 & $01-12-76$ & 177 \\
\hline $00 \Delta N=015 E-11$ & $B O A$ & 1 & OGALLALA & FORMATION & $01-22-75$ & 161.15 & -1.03 & $01-15-70$ & 102.18 \\
\hline $004 N-015 E-15$ & $C A B$ & 1 & OGALLALA & FUKMATIUN & $01-22-75$ & 18 & -0.01 & $01-12-70$ & 181.01 \\
\hline $004 N=015 E-17$ & $\Delta 80$ & 1 & $O G \Delta L L A L A$ & FORMATIUN & $01-22-75$ & 203.22 & -0.20 & $01-12-76$ & 209.42 \\
\hline $004 N=015 E-25$ & Dob & 1 & GGALLALA & FORMATIUN & $01-23-75$ & 148.97 & -3.13 & $01-15-70$ & 152.10 \\
\hline $004 N-115 E-20$ & $A C C$ & 1 & CUGALLALA & FORMATIUN & $01-22-75$ & 167.00 & -17.10 & $01-12-76$ & 184.16 \\
\hline
\end{tabular}


Table 1.--Water levels and water-level changes--Continued.

IEXAS COUNTY

\begin{tabular}{|c|c|c|c|c|c|c|c|c|c|}
\hline NELL & & & PRIN & NCIPAL & $\begin{array}{r}\text { WATER } \\
197\end{array}$ & LEVEL & $\begin{array}{l}\text { NATER } \\
\text { LEVEL }\end{array}$ & $\begin{array}{r}\text { WATER } \\
197\end{array}$ & LEVEL \\
\hline LOCATIUN & & & $A O U$ & UIFER & DATE & DEPTH & $\begin{array}{l}\text { CHANGE } \\
1975-76\end{array}$ & DATE & UEPTH \\
\hline $004 N=010 E=05$ & $C C \theta$ & 1 & OGALLALA & FURMATION & $01-23-75$ & 133.04 & -4.10 & $01-14-76$ & 137.14 \\
\hline $004 N=010 E=17$ & BAU & 1 & OGALLALA & FORMATION & $01-23-75$ & 110.11 & 4.82 & $01-14-76$ & 105.29 \\
\hline $004 N-016 E-22$ & DCO & 1 & OGALLALA & FORMATIUN & $01-23-75$ & 116.52 & 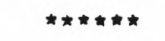 & $01-14-76$ & 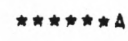 \\
\hline $004 N-016 E-29$ & 880 & 1 & UGALLALA & FURMATION & $01-23-75$ & 120.48 & -1.72 & $01-14-70$ & 130.20 \\
\hline $004 N=017 E-03$ & $\mathrm{CBC}$ & 1 & OGALLALA & FORMATIUN & $01-23-75$ & 121.03 & -1.42 & $01-14-76$ & 123.05 \\
\hline $004 N-017 E-14$ & 600 & 1 & UGALLALA & FORMATION & $01-23=75$ & 140.26 & 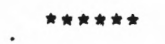 & $01-15-76$ & $\star \star \star \star \star \star \star \star A A$ \\
\hline $004 N=017 E-21$ & $A A A$ & 1 & OGALLALA & FURMAIION & $01-23-75$ & 156.50 & 0.90 & $01-15-76$ & 155.60 \\
\hline $004 N=017 E-23$ & $A \Delta A$ & 1 & OGALLALA & FORMATION & $01-23-75$ & 130.04 & -7.74 & $01-14-70$ & 137.78 \\
\hline $004 N-017 E-33$ & $\triangle D D$ & 1 & OGALLALA & FURMATION & $01-23-75$ & 141.02 & -3.53 & $01-14-76$ & 144.55 \\
\hline $004 N=018 E-13$ & CCO & 1 & OGALLALA & FURMATION & $01-23-75$ & 110.30 & 0.04 & $01-15-70$ & 116.26 \\
\hline $00414=018 \varepsilon-25$ & $\cos$ & 1 & OGALLALA & FORMATION & $01-23-75$ & 123.25 & 14.95 & $01-14-76$ & 108.30 \\
\hline $004 N=018 E=26$ & $B C A$ & 1 & OGALLALA & FOKMATIUN & $01-23-75$ & 110.51 & 0.38 & $01-15-70$ & 110.13 \\
\hline $004 N=019 E-22$ & CBC & 1 & OGALLALA & FORMATIUN & $01-23-75$ & 98.65 & -5.10 & $01-15-76$ & 103.75 \\
\hline $004 N=019 E-27$ & $\triangle D C$ & 1 & OGALLALA & FORMATION & $01-22-75$ & 94.91 & $-25.72 C$ & $01-15-76$ & $120.63 C$ \\
\hline $004 N=019 E-27$ & $\Delta 00$ & 1 & DGALLALA & FURMATIUN & $01-22-75$ & 94.25 & $-16.18 \mathrm{C}$ & $01-15-76$ & 110.436 \\
\hline $005 N=010 E=07$ & $B C C$ & 1 & OGALLALA & FURMATIUN & $01-21-75$ & 217.50 & -1.04 & $01-13-76$ & 218.54 \\
\hline $005 N-011 E-10$ & 368 & 1 & DGALLALA & FURMATION & $01-21-75$ & 227.78 & -1.71 & $01-13-76$ & 229.49 \\
\hline $005 N-01<E-00$ & DAA & 1 & IJGALLALA & FURMATIUN & $01-21-75$ & 235.00 & 1.20 & $01-13-76$ & 234.40 \\
\hline $005 N-012 E-10$ & $\triangle B O$ & 1 & DGALLALA & FORMATION & $01-21-75$ & 226.50 & 0.74 & $01-13-76$ & 225.76 \\
\hline $005 i N-013 E-01$ & CBA & 1 & OGALLALA & FORMATIUN & $01-22-75$ & 200.90 & -2.84 & $01-14-76$ & 269.74 \\
\hline $005 N=013 E=04$ & $C 8 B$ & 1 & DGALLALA & FORMATION & $01-22-75$ & 267.08 & -3.78 & $01-14-76$ & 270.80 \\
\hline $005 N=013 E-08$ & 880 & 1 & OGALLALA & FORMATIUN & $01-22-75$ & $\star \star \star \star \star \star \star A$ & 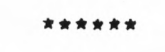 & $01-14-76$ & $x$ \\
\hline $05 N-013 E-14$ & vots & 1 & UGALLALA & FORMATION & $01-22-75$ & 241.48 & -3.41 & $01-14-76$ & 244.89 \\
\hline
\end{tabular}


Table 1.-Water levels and water-level changes--Continued.

\begin{tabular}{|c|c|c|c|c|c|c|c|c|c|}
\hline \multirow{2}{*}{$\begin{array}{c}\text { WELL } \\
\text { LOCATION }\end{array}$} & \multirow{2}{*}{ 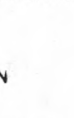 } & \multirow{2}{*}{\multicolumn{3}{|c|}{$\begin{array}{l}\text { PRINCIPAL } \\
\text { AUUIFER }\end{array}$}} & \multicolumn{2}{|c|}{$\begin{array}{l}\text { WATER LEVEL } \\
1975\end{array}$} & \multirow{2}{*}{$\begin{array}{l}\text { WATER } \\
\text { LEVEL } \\
\text { CHANGE } \\
1975-70\end{array}$} & \multicolumn{2}{|c|}{$\begin{array}{l}\text { WATEK LEVEL } \\
1976\end{array}$} \\
\hline & & & & & DATE & DEPTH & & DATE & LEPTH \\
\hline $005 N-014 E-01$ & $\triangle 68$ & 1 & OGALLALA & FURMATIUN & $01-21-75$ & 228.80 & -2.00 & $01-14-76$ & 230.80 \\
\hline $005 N=014 E-10$ & $\mathrm{CBO}$ & 1 & UGALLALA & FORMATIUIV & $01-22-75$ & 235.74 & -5.27 & $01-14-76$ & 241.01 \\
\hline $005 N=014 E-31$ & $\triangle C C$ & 1 & DGALLALA & FORMATIUN & $01-22-75$ & 188.80 & -4.23 & $01-14-76$ & 193.03 \\
\hline $005 N-015 E-00$ & COO & 1 & OGALLALA & FURMATION & $01-22-75$ & 191.98 & -3.06 & $01-14-76$ & 195.04 \\
\hline $005 N=015 E-11$ & $C A D$ & 1 & UGALLALA & FORMATIUN & $01-22-75$ & 186.30 & -5.09 & $01-14=76$ & 191.99 \\
\hline $005 N=015 E-14$ & $C \forall A$ & 1 & OGALLALA & FORMATION & $01-22-75$ & 166.94 & -4.04 & $01-16-70$ & 170.98 \\
\hline $005 N-015 E-20$ & DAB. & 1 & OGALLALA & FORMATION & $01-22-15$ & 160.18 & -2.82 & $01-14-70$ & 163.00 \\
\hline $005 N=016 E-05$ & $\triangle A A$ & 1 & OGALLALA & FORMATION & $01-22-75$ & 140.40 & 0.00 & $01-15-76$ & 140.40 \\
\hline $005 \mathrm{~N}-010 E-18$ & ODC & 2 & DGALLALA & FORMATILIN & $01-22-75$ & 138.00 & $\hbar * \star \star * *$ & $01-10-70$ & 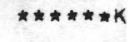 \\
\hline $005 N-017 E=01$ & $A D A$ & 1 & OGALLALA & FORMATIUN & $01-23-75$ & 81.50 & 0.30 & $01-16-76$ & 81.20 \\
\hline $005 N=1) 17 E=05$ & $\triangle 00$ & 1 & OGALLALA & FORMATION & $01-23-75$ & 101.53 & 0.51 & $01-16-76$ & 101.02 \\
\hline $005 N-017 E-09$ & ODA & 1 & UGALLALA & FORMATION & $01-23-75$ & 108.25 & 1.12 & $01-16-76$ & 107.13 \\
\hline $005 N-017 E-11$ & DCC & 1 & OGALLALA & FORMATION & $01-23-75$ & 102.26 & -1.15 & $01-16-76$ & 103.41 \\
\hline $005 N-017 E-17$ & $\operatorname{CsA}$ & 1 & OGALLALA & FORMATIUN. & $01-23-75$ & 124 & -0.20 & $1-16-76$ & 12 \\
\hline $005 N-017 E-27$ & $A B C$ & 1 & OGALLALA & FURMATIUN & $01-23-75$ & 115.01 & -2.82 & $01-10-76$ & 118.03 \\
\hline $005: V=018 E-02$ & Des & 1 & OGALLALA & FURMATIUN & $01-23-75$ & 100.42 & -22.68 & $01-10-76$ & 123.10 \\
\hline $005 N-019 E-04$ & VOA & 1 & UGALLALA & FUKMATION & & * & $\star \star \star *$ & & $* * * * * *$ \\
\hline $005 N=019 E-14$ & 804 & 1 & UGALLALA & FORMATION & & $\star \star$ & $\not \star \star \star \star \star * *$ & & 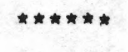 \\
\hline $005 N-019 E-29$ & DAA & 1 & OGALLALA & FURMATION & & & 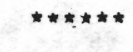 & & \\
\hline $00 O N=010 E=10$ & Hot & 1 & OGALLALA & FORMATION & $01-21-75$ & 194.00 & 0.17 & $01-14-70$ & .89 \\
\hline $0 O B N=010 E-23$ & $A \cup A$ & 1 & OGALLALA & FURMATION & $01-21-75$ & 245.70 & -0.70 & $01-14-76$ & 246.40 \\
\hline $000 N=010 E-30$ & $\operatorname{lcc}$ & $l$ & OGALLALA & FURMATION & $01-21-75$ & 224.00 & -13.00 & $01-13-76$ & 237.00 \\
\hline $00 O N=010 E-30$ & vos & 1 & OGALLALA & FORMATIUN & $01-21-75$ & 280.90 & -0.72 & $01-13-76$ & 289.62 \\
\hline
\end{tabular}


Table 1.--Water levels and water-level changes--Continued.

TEXAS COUNTY

\begin{tabular}{|c|c|c|c|c|c|c|c|c|c|}
\hline & & & PRIN & NCIPAL & $\begin{aligned} \text { WATER } \\
197\end{aligned}$ & $\begin{array}{l}\text { LEVEL } \\
5\end{array}$ & $\begin{array}{l}\text { WATER } \\
\text { LEVEL }\end{array}$ & $\begin{array}{l}\text { WATER } \\
197\end{array}$ & $\begin{array}{l}\text { LEVEL } \\
\sigma\end{array}$ \\
\hline LOCATIOA & & & $\Delta Q C$ & UIFER & DATE & DEPIH & $\begin{array}{r}\text { CHANGE } \\
1975-76\end{array}$ & DATE & UEPTH \\
\hline $006 N=011 E-17$ & $C B C$ & 1 & OGALLALA & FURMATIUN & $01-21-75$ & $\star \star \star \star \star \star \star \star A$ & 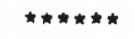 & $01-13-76$ & 284.86 \\
\hline $006 N-011 E-25$ & $B C C$ & 1 & OGALLALA & FORMAIIUN & $01-21-75$ & 276.31 & -0.47 & $01-13-76$ & 276.78 \\
\hline $006 N-011 E-20$ & $\Delta C \theta$ & 2 & OGALLALA & FURMATIUN & $01-21-75$ & 273.80 & -0.04 & $01-13-70$ & 273.84 \\
\hline $000 N=012 E-21$ & $\operatorname{ccc}$ & 1 & OGALLALA & FORMATION & $01-21-75$ & $\star \star \star \star \star \star \star \star \star \Delta$ & 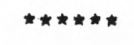 & $01-13-70$ & 270.24 \\
\hline $00 O N=012 E-36$ & $C C C$ & 1 & UGALLALA & FORMATIUN & $01-21-75$ & 236.75 & -4.94 & $01-13-7 b$ & 241.69 \\
\hline $006 N=013 E-16$ & $A C C$ & 1 & OGALLALA & FORMATION & $01-25-75$ & 264.70 & -10.78 & $01-14-70$ & 275.48 \\
\hline $00 B N=013 E-20$ & 004 & 1 & DGALLALA & FORMATION & $01-21-75$ & 294.22 & -3.32 & $01-14=76$ & 297.54 \\
\hline $006 N=013 t-30$ & $\cos$ & 1 & DGALLALA & FORMATIUN & $01-21-75$ & 247.50 & -3.50 & $01-14-76$ & 251.00 \\
\hline $000 N=014 E-21$ & $A C d$ & 1 & DGALLALA & FURMATION & $01-22-75$ & 232.50 & -4.40 & $01-14-76$ & 230.90 \\
\hline $006 N-014 E-33$ & OAB & 1 & OGALLALA & FURMATIUN & $01-22-75$ & 257.30 & -5.71 & $01-14-76$ & 263.01 \\
\hline $006 N=014 E-35$ & $\cos$ & 1 & OGALLALA & FORMATION & $01-22-75$ & 245.10 & -1.40 & $01-14-76$ & 246.50 \\
\hline $006 N=015 E-14$ & $\triangle A A$ & 1 & OGALLALA & FURMATIUN & $01-22-75$ & 124.43 & -3.65 & $01-16-76$ & 128.08 \\
\hline $000 N=015 E-29$ & 868 & 2 & OGALLALA & FURMATION & $01-22-75$ & 113.62 & -4.60 & $01-14-76$ & 118.22 \\
\hline $000 N-015 E=30$ & $\triangle C C$ & 1 & OGALLALA & FURMATION & $01-22-75$ & 196.21 & -4.99 & $01-14-76$ & 201.20 \\
\hline $000 N-010 E-15$ & 000 & 1 & UGALLALA & FORMATION & $01-22-75$ & 90.68 & -2.05 & $01-14-76$ & 92.73 \\
\hline $006 N-U 10 E-20$ & $B A A$ & 1 & OGALLALA & FORMATION & $01-21-75$ & 133.90 & -0.14 & $01-16-76$ & 134.04 \\
\hline$J 00 N-016 E-27$ & $A C C$ & 1 & DGALLALA & FORMATION & $01-23-75$ & 86.03 & -2.53 & $01-16-76$ & 88.50 \\
\hline $300 N-017 E-14$ & DBE & 1 & OGALLALA & FURMATION & $01-23-75$ & $\star \star \star \star \star \star \star \star \hbar k$ & 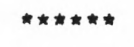 & $01-16-76$ & 135.76 \\
\hline $300 N-017 E-23$ & $C B C$ & 1 & OGALLALA & FURMATION & $01-23-75$ & 102.69 & 4.67 & $01-16-70$ & 98.02 \\
\hline $200 N-017 E-5 O$ & CCO & 1 & OGALLALA & FORMATIUN & $01-23-75$ & 102.56 & -3.26 & $01-16-76$ & 105.82 \\
\hline $300 N-017 E-34$ & 000 & 1 & UGALLALA & FORMATION & $01-23-75$ & 89.76 & -1.03 & $01-16-76$ & 91.39 \\
\hline $106 N=018 E=09$ & $D \triangle C$ & 1 & DGALLALA & FORMATION & $01-23-75$ & 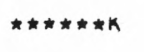 & 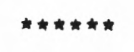 & $01-16-76$ & $m \times$ \\
\hline $100 N-010 E-28$ & coo & 1 & OGALLALA & FORMATION & $01-23-75$ & 75.41 & -2.09 & $01-16-76$ & 77.50 \\
\hline
\end{tabular}


Table 1.-Water levels and water-level changes--Continued.

PELL
LOCATIUN
AQUIFER


Table 1.-Water levels and water-level changes-.-Continued.

TILLMAN COUNTY

\begin{tabular}{|c|c|c|c|c|c|c|c|c|c|}
\hline \multirow{2}{*}{\multicolumn{2}{|c|}{$\begin{array}{c}\text { WELL } \\
\text { LOCATIUN }\end{array}$}} & \multirow{2}{*}{\multicolumn{3}{|c|}{$\begin{array}{l}\text { PRINCIPAL } \\
\text { AGUIFER }\end{array}$}} & \multicolumn{2}{|c|}{$\begin{array}{l}\text { WATER LEVEL } \\
1975\end{array}$} & \multirow{2}{*}{$\begin{array}{l}\text { WATER } \\
\text { LEVEL } \\
\text { CHANGE } \\
1975-70\end{array}$} & \multicolumn{2}{|c|}{$\begin{array}{l}\text { WATER LEVEL } \\
1976\end{array}$} \\
\hline & & & & & DATE & DEPTH & & DATE & OEPTH \\
\hline $001 s-017 w=06$ & CDO & 1 & TERRACE & DEPOSITS & $12-09-74$ & 16.43 & 0.98 & $12-10-75$ & 15.45 \\
\hline $0015-018 n=01$ & OAA & 1 & TERRACE & OEPOSITS & $12-09-74$ & 25.34 & 1.72 & $12-10-75$ & 23.62 \\
\hline $0015-018 w=04$ & $U C D$ & 1 & TERRACE & DEPOSITS & $12-09-74$ & 12.99 & 2.80 & $12-10-75$ & 10.19 \\
\hline $0015=010 w=05$ & 080 & 1 & TERRACE & DEPOSITS & $12-09-74$ & 26.42 & 3.33 & $12-10-75$ & 23.09 \\
\hline $0015-018 w-10$ & DCC & 1 & TERRACE & DEPOSITS & $12-09-74$ & 26.36 & 2.52 & $12-10-75$ & 23.84 \\
\hline $0015-018 w-14$ & $\cos$ & 1 & TERRACE & OEPOSITS & $12-09-74$ & 23.21 & 4.00 & $12-10-75$ & 19.21 \\
\hline $0015-018 w-15$ & CAA & 1 & TERRACE & DEPOSITS & $12-09-74$ & 35.63 & 2.79 & $12-10-75$ & 32.84 \\
\hline $0015=018 m-15$ & DCC & 1 & TERRACE & DEPOSIIS & $12-09-74$ & 21.93 & 3.35 & $12=09-75$ & 18.58 \\
\hline $0015=018 k-19$ & $A 00$ & 1 & TERRACE & UEPOSITS & $12-10-74$ & 18.25 & 4.25 & $12-10-75$ & 14.00 \\
\hline $0015-018 w-20$ & DAO & 1 & TERRACE & DEPUSITS & $12-10-74$ & 23.97 & 2.64 & $12-10-75$ & 21.33 \\
\hline $001 s-016 w-21$ & $\triangle B B$ & 1 & TERRACE & DEPOSITS & $12-10=74$ & 13.00 & 1.37 & $12-10-75$ & 11.63 \\
\hline $0015-018 w-22$ & $\triangle 00$ & 1 & TERRACE & DEPUSITS & $12-09-74$ & 24.50 & 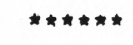 & & $\|* * *\| *$ \\
\hline $0015-118 w-22$ & $B C B$ & 1 & TERKACE & DEPOSITS & $12-09-74$ & 34.55 & 3.53 & $12-09-75$ & 31.02 \\
\hline $0015-016 w-23$ & $B A A$ & 1 & TERRACE & OEPUSITS & $12-09-74$ & 24.66 & 2.93 & $12-09-75$ & 21.73 \\
\hline $001 s-0181 N-23$ & $\operatorname{ccc}$ & 1 & TERRACE & DEPUSITS & $12-09-74$ & 26.52 & 2.72 & $12-10-75$ & 23.80 \\
\hline $0013-018 n-25$ & AOA & 1 & TERRACE & DEPOSITS & $12-10-74$ & 13.70 & 2.05 & $12-10-75$ & 11.65 \\
\hline $0015-018 w-20$ & $\triangle A A$ & 1 & TERRACE & DEPOSIIS & $12-10-74$ & 29.40 & 0.72 & $12-10-75$ & 28.68 \\
\hline $0015-018 w-20$ & $\forall \otimes C$ & 1 & TERRACE & CEPUSITS & $12-09-74$ & 24.78 & 2.33 & $12-10-75$ & 22.45 \\
\hline $0013=018 n-26$ & $C C A$ & 1 & TERRACE & DEPUSITS & $12=10-74$ & 24.05 & -1.22 & $12-10-75$ & 25.27 \\
\hline $0015-018 m-27$ & OAA & $\downarrow$ & TERRACE & DEPLSITS & $12-10-74$ & 20.59 & 3.21 & $12-10-75$ & 25.38 \\
\hline $001 s=018 N-27$ & 580 & 3 & TERKACE & DEPDSITS & $12-10-74$ & 29.40 & 3.13 & $12-10=75$ & 26.27 \\
\hline $0015=018 w-25$ & SAA & 1 & TERRACE & DEPOSITS & $12-10=74$ & 17.90 & 1.70 & $12-10=75$ & 16.20 \\
\hline $001 s-018 w-29$ & $\forall+0$ & 1 & TERRACE & DEPOSITS & $12-10-74$ & 28.02 & 1.45 & $12-10-75$ & 26.57 \\
\hline
\end{tabular}


Table 1.-Water levels and water-level changes--Continued.

TILLMAN CIJUNTY

\begin{tabular}{|c|c|c|c|c|c|c|c|c|c|}
\hline \multirow{2}{*}{$\begin{array}{c}\text { WELL } \\
\text { LOCATIUN }\end{array}$} & \multirow{2}{*}{ 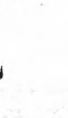 } & \multirow{2}{*}{\multicolumn{3}{|c|}{$\begin{array}{l}\text { PRINCIPAL } \\
\text { AQUIFER }\end{array}$}} & \multicolumn{2}{|c|}{$\begin{array}{c}\text { WATER LEVEL } \\
1975\end{array}$} & \multirow{2}{*}{$\begin{array}{l}\text { WATER } \\
\text { LEVEL } \\
\text { CHANGE } \\
1975=76\end{array}$} & \multicolumn{2}{|c|}{$\begin{array}{c}\text { WATER LEVEL } \\
1976\end{array}$} \\
\hline & & & & & DATE & DEPTH & & DATE & DEPIH \\
\hline $0015=018 w-33$ & $A A A$ & 7 & TERFACE & DEPOSITS & $12-10=74$ & 25.15 & 0.75 & $12-10-75$ & 24.40 \\
\hline $001 s-018 w-33$ & CDO & 8 & TERRACE & OEPOSITS & $12-10-74$ & 29.35 & -0.15 & $12-10-75$ & 29.50 \\
\hline $0015-018 m-33$ & DAB & 5 & TERRACE & UEPOSITS & $12-10-74$ & $\star \star \star \star \star \star \star \star k$ & $\star \star \star \star \star \star \star$ & $12-10-75$ & 22.80 \\
\hline $0015=010 \mathrm{w}=35$ & $\Delta \otimes C$ & 1 & TERRACE & DEPOSITS & $12-10-74$ & 29.75 & 2.95 & $12-10-75$ & 26.80 \\
\hline $001 s-018 w=36$ & $B C C$ & 1 & TERRACE & DEPOSITS & $12-10-74$ & 29.02 & 1.39 & $12-10-75$ & 28.43 \\
\hline $0015=019 n 01$ & $\operatorname{coc}$ & 1 & TERRACE & DEPOSITS & $12-12-74$ & 41.36 & 1.97 & $12=09-75$ & 39.39 \\
\hline $0015-019 w-02$ & $\triangle \cup C$ & 9 & TERRACE & DEPOSITS & $12-12-74$ & 40.07 & 2.95 & $12=09-75$ & 37.72 \\
\hline $0015-019 w-02$ & $\triangle A C$ & 4 & TERRACE & DEPOSITS & $12-12-74$ & 37.05 & 1.93 & $12-09-75$ & 35.72 \\
\hline $0015-019 w=02$ & $\operatorname{COO}$ & 8 & TERRACE & DEPOSITS & $12-12-74$ & $\star \star \star \star \star \star \star \star k K$ & 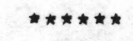 & $12-09-75$ & 38.74 \\
\hline $0015=019 w=03$ & $\triangle A U$ & 1 & TERRACE & DEPUSITS & $12-12-74$ & 32.91 & 1.31 & $12-09-75$ & 31.60 \\
\hline $0015-019 m=03$ & $D A B$ & 2 & TERRACE & DEPOSITS & $12-12-74$ & $\hbar \star \star * * * \hbar k$ & 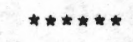 & $12-09=75$ & 31.45 \\
\hline $001 s=019 m=04$ & UAU & 1 & TERRACE & DEPUSITS & $12-12-74$ & 10.28 & 2.34 & $12-09-75$ & 7.94 \\
\hline $0015=019 n=05$ & DAU & 1 & TERRACE & DEPDSITS & $12-12-74$ & 0.50 & 2.07 & $12-09-75$ & 0.49 \\
\hline $001 s=019 w-08$ & $A O A$ & 1 & TERRACE & DEPUSITS & $12-12-74$ & 9.13 & 1.04 & $12=09-75$ & 8.09 \\
\hline $0015-019 w=09$ & HEA & 1 & TERRACE & DEPOSITS & $12-12-74$ & 11.83 & 2.15 & $12=09-75$ & 9.68 \\
\hline $0015-019 m-10$ & $\Delta A \theta$ & 2 & TERRACE & DEPUSITS & $12-12-7 a$ & 32.18 & 2.52 & $12-09-75$ & 29.66 \\
\hline $0015-019 w-10$ & DDA & 4 & TERRACE & OEPUSITS & $12-12-74$ & 36.58 & -2.34 & $12-11-75$ & 38.92 \\
\hline $0015-019 w-11$ & $A A B$ & 1 & TERRACE & DEPOSITS & & 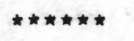 & $\star x$ & $12=09-75$ & 39.95 \\
\hline $001 s-019 w-11$ & DoC & 2 & TERRACE & DEPOSITS & & 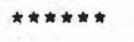 & $\star \star \star \star \star \star \star * *$ & $12-09-75$ & 40.08 \\
\hline $0015-019 m-12$ & $\triangle C A$ & 2 & TERRACE & DEPUSITS & & $\star \star \star \star \star \star \star \star$ & $\star \star \star \star \star \star \star \star$ & $12-09-75$ & 39.29 \\
\hline $0015-019 m-13$ & occ & 1 & TERRACE & DEPUSITS & $12-10-74$ & 29.05 & 2.37 & $12-09-75$ & 26.08 \\
\hline $0015=019 x-14$ & OAD & 1 & TERRACE & DEPUSITS & $12-12-74$ & 20.10 & 1.71 & $12-09-75$ & 20.39 \\
\hline $0015-019 n-14$ & NDD & 1 & TERRACE & DEPUSITS & $12-12-74$ & 27.10 & -2.04 & $12-09-75$ & 29.14 \\
\hline
\end{tabular}


Table 1.-Water levels and water-level changes--Continued.

IILLMAN COUNTY

\begin{tabular}{|c|c|c|c|c|c|c|c|c|c|}
\hline WELL & & & $P R$ & INCIPAL & $\begin{array}{r}\text { WATER } \\
197\end{array}$ & EVEL & $\begin{array}{l}\text { WATER } \\
\text { LEVEL }\end{array}$ & $\begin{array}{r}\text { WATER } \\
197\end{array}$ & EVEL \\
\hline LOCATIUR & & & & WUIFEK & DATE & DEPTH & CHANGE & DATE & DEFTH \\
\hline $0015-019 w-15$ & $A \Delta A$ & 1 & TERRACE & DEPOSIIS & $12-12-74$ & 35.80 & 0.14 & $12-09-75$ & 35.72 \\
\hline $0015-019 w-15$ & 386 & 2 & TEKRACE & DEPUSITS & $12-12-74$ & 31.06 & 4.80 & $12-09-75$ & 26.26 \\
\hline $0015-019 n-15$ & 000 & 4 & TERRACE & UEPISITS & $12-12-74$ & 30.93 & 2.55 & $12-09-75$ & 34.38 \\
\hline $0015=019 w-16$ & טOC & 1 & TERRACE & DEPOSITS & $12-12-74$ & 23.62 & 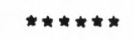 & $12-10-75$ & 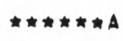 \\
\hline $001 s-019 w-20$ & $D A \Delta$ & 1 & TERRACE & DEPUSITS & $12-12-74$ & 13.79 & 1.37 & $12-10-75$ & 12.42 \\
\hline $0015=019 w-21$ & $\forall \forall A$ & 1 & TERRACE & DEPOSITS & $12-12-74$ & 12.39 & 2.47 & $12-10-75$ & 9.92 \\
\hline $0015-019 m-21$ & $c \in 0$ & 2 & TERRACE & DEPOSITS & & $\| \star \star \star \star \star \star \star$ & 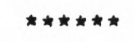 & $12-10-75$ & 6.15 \\
\hline $0015-019 w-22$ & $\Delta A U$ & 7 & TERRACE & DEPOSITS & $12-12-74$ & 33.25 & 2.43 & $12-10-75$ & 30.82 \\
\hline $0015=019 \mathrm{~m}-22$ & 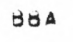 & 8 & TERRACE & DEPOSITS & $: 2-12-74$ & 36.46 & 2.93 & $12-10-75$ & 33.53 \\
\hline $0015-019 w-22$ & $\operatorname{CBA}$ & 0 & TERKACE & DEPOSITS & $12-12-74$ & 37.07 & 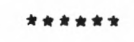 & $12-10-75$ & $\star \star \star \star \star \star$ \\
\hline $0015-019 n-23$ & $A A \Delta$ & 1 & TERRACE & DEPOSITS & $12-12-74$ & 20.44 & 1.59 & $12-09-75$ & 24.85 \\
\hline $0015-019 w=24$ & $A A C$ & 1 & TERRACE & DEPUSITS & $12-12-74$ & 30.02 & 2.54 & $12-09-75$ & 28.08 \\
\hline $0015-019 n-24$ & $\triangle 00$ & 2 & TERRACE & UEPOSITS & $12-10-74$ & 28.18 & 1.38 & $12-09-75$ & 26.80 \\
\hline $0015=019 m-25$ & DAO & 1 & TERRACE & OEPOSITS & $12-10-74$ & 27.62 & 8.23 & $12-09-75$ & 19.39 \\
\hline $0015-019 n-20$ &  & 1 & TERRACE & DEPUSITS & & \# * & $\star \star \star \star \star \star \star$ & $12-10-75$ & 22.99 \\
\hline $0015-019 w-27$ & $\operatorname{Aag}$ & 1 & TERRACE & DEPUSITS & & 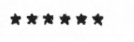 & $\star \star \star \star \star \star \star$ & $12-10-75$ & 26.06 \\
\hline $0015-019 w=20$ & $\cos$ & 2 & TERRACE & DEPOSITS & $12-10-74$ & 9.55 & 1.75 & $12-10-75$ & 7.80 \\
\hline $0015=019 w-28$ & 000 & 1 & TERRACE & DEPOSITS & $12-10-74$ & 15.87 & 4.22 & $12-10=75$ & 11.65 \\
\hline $0015-019 w-20$ & $A A A$ & 1 & TERRACE & DEPOSITS & $12-10=74$ & $\star \star \star \star \star \star \star \star k$ & 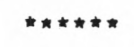 & $12-10-75$ & 10.18 \\
\hline $0015-019 m-29$ & 08A & 2 & TERRACE & DEPOSITS & $12-10-74$ & 9.00 & 0.70 & $12-10-75$ & 8.30 \\
\hline $0015-019 m-29$ & $D O C$ & 3 & TERRACE & DEPUSITS & $12-12-74$ & 10.32 & 2.82 & $12-10-75$ & 7.50 \\
\hline $001 s-019 w-33$ & Cos & 2 & TERRACE & DEPOSITS & $12-10-74$ & $\star \# \# \# \# k$ & 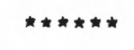 & $12-10-75$ & 5.90 \\
\hline $0015=019 m=33$ & ODA & 1 & TERRACE & DEPOSITS & $12-10-74$ & 16.73 & 0.35 & $12-10-75$ & 16.38 \\
\hline
\end{tabular}


Table 1.--Water levels and water-level changes--Continued.

TILLMAN COUNTY

\begin{tabular}{|c|c|c|c|c|c|c|c|c|c|}
\hline \multirow{2}{*}{ LOCATION } & \multirow{2}{*}{ 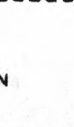 } & \multirow{2}{*}{\multicolumn{3}{|c|}{$\begin{array}{l}\text { PRINCIPAL } \\
\text { ABUIFER }\end{array}$}} & \multicolumn{2}{|c|}{$\begin{array}{c}\text { WATER LEVEL } \\
1975\end{array}$} & \multirow{2}{*}{$\begin{array}{l}\text { WATER } \\
\text { LEVEL } \\
\text { CHANGE } \\
1975=76\end{array}$} & \multicolumn{2}{|c|}{$\begin{array}{l}\text { WATER LEVEL } \\
1976\end{array}$} \\
\hline & & & & & DATE & DEPTH & & OATE & DEPTH \\
\hline $0015=019 w-34$ & $B A C$ & 1 & TERRACE & DEPUSITS & $12-10-74$ & 20.18 & 3.01 & $12-10-75$ & 16.57 \\
\hline $0015-019 w-35$ & $A D A$ & 1 & TERRACE & DEPOSITS & $12-10-74$ & $\star \star \star \star \star \star \star \star k$ & 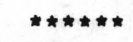 & $12-09-75$ & 26.00 \\
\hline $0015=019 w-36$ & CBE & 1 & TERRACE & VEPOSITS & $12-10-74$ & 32.25 & 1.39 & $12-09-75$ & 30.86 \\
\hline $0.02 s-018 m-02$ & $\cot$ & 1 & TERRACE & DEPOSITS & $12-10-74$ & 33.09 & 1.65 & $12-11-75$ & 31.44 \\
\hline $002 s-018 m-03$ & 000 & 1 & TERRACE & DEPOSITS & $12-09=74$ & 33.72 & 1.49 & $12-11-75$ & 32.23 \\
\hline $0025-018 w-04$ & $\triangle C C$ & 3 & TERRACE & DEPUSITS & $12-10-74$ & 47.78 & 0.58 & $12-11-75$ & 47.20 \\
\hline $0025-018 w-04$ & $D C \theta$ & 2 & TERRACE & DEPDSITS & & $\hbar \star \star \star \star * \star *$ & 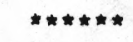 & $12-11-75$ & 54.65 \\
\hline $0025=018 w-04$ & $O C D$ & 1 & TERRACE & DEPOSITS & $01-28-75$ & 49.72 & 0.65 & $12-11-75$ & 49.07 \\
\hline $002 s-018 m-0 s$ & DAO & 1 & TERRACE & DEPUSITS & $12-10=74$ & 74.34 & -2.68 & $12-11-75$ & 77.02 \\
\hline $002 s-010 w=00$ & $\forall C B$ & 2 & TERRACE & DEPOSITS & $12-10-74$ & 13.30 & -4.23 & $12-11-75$ & 17.53 \\
\hline $0025-018 n=09$ & $B \forall A$ & 4 & TERRACE & DEPOSITS & $12-09-74$ & 63.29 & 0.09 & $12-11-75$ & 63.20 \\
\hline $0025=018 w-09$ & COU & 5 & TERRACE & DEPUSITS & $12-10-74$ & 60.15 & 0.19 & $12-11-75$ & 59.96 \\
\hline $0025-018 m-11$ & $B \forall A$ & 1 & TERRACE & DEPUSITS & $12-10-74$ & 31.02 & 1.59 & $12-11-75$ & 29.43 \\
\hline $0025=018 w-15$ & $\Delta \forall A$ & 1 & TERRACE & DEPOSITS & $12-10-74$ & 38.68 & 0.80 & $12-11-75$ & 37.88 \\
\hline $0025-018 m-15$ & $8 \mathrm{Cs}$ & 4 & TERRACE & OEPOSITS & & 中* & 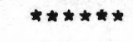 & $12-11-75$ & 40.42 \\
\hline $0025-018 w-15$ & c6u & 3 & TERRACE & DEPDSITS & $12-10-74$ & 45.05 & 0.23 & $12-11-75$ & 44.92 \\
\hline $0025=018 w-15$ & OCC & 2 & TERRACE & DEPDSITS & $12-10-74$ & 47.99 & 2.15 & $12-11-75$ & 45.84 \\
\hline $0025-018 w-10$ & $B A A$ & 1 & TERRACE & DEPOSITS & $12-10=74$ & 49.22 & 1.13 & $12-11-75$ & 48.09 \\
\hline $0025-018 w-19$ & $\mathrm{CCB}$ & 1 & TERRACE & DEPOSITS & $12-11-74$ & 41.47 & 0.67 & $12-11-75$ & 40.80 \\
\hline $002 s-018 m-22$ & $D A B$ & 1 & TERRACE & DEPOSITS & $12-10-74$ & 35.92 & 0.92 & $12-11-75$ & 35.00 \\
\hline $0025=018 m-23$ & $A B E$ & 1 & TERRACE & DEPUSITS & $12-10-74$ & 11.21 & 0.69 & $12-11-75$ & 10.52 \\
\hline $0025-018 N-29$ & DOB & 1 & TERRACE & DEPOSITS & $12-11-74$ & 27.63 & 3.09 & $12-11-75$ & 24.54 \\
\hline $0023-018 w-34$ & $\triangle A C$ & 1 & TERRACE & DEPOSITS & $12-10-74$ & 22.02 & 0.72 & $12-11-75$ & 21.90 \\
\hline
\end{tabular}


Table 1.--Water levels and water-level changes--Continued.

TILLMAN CDUNTY

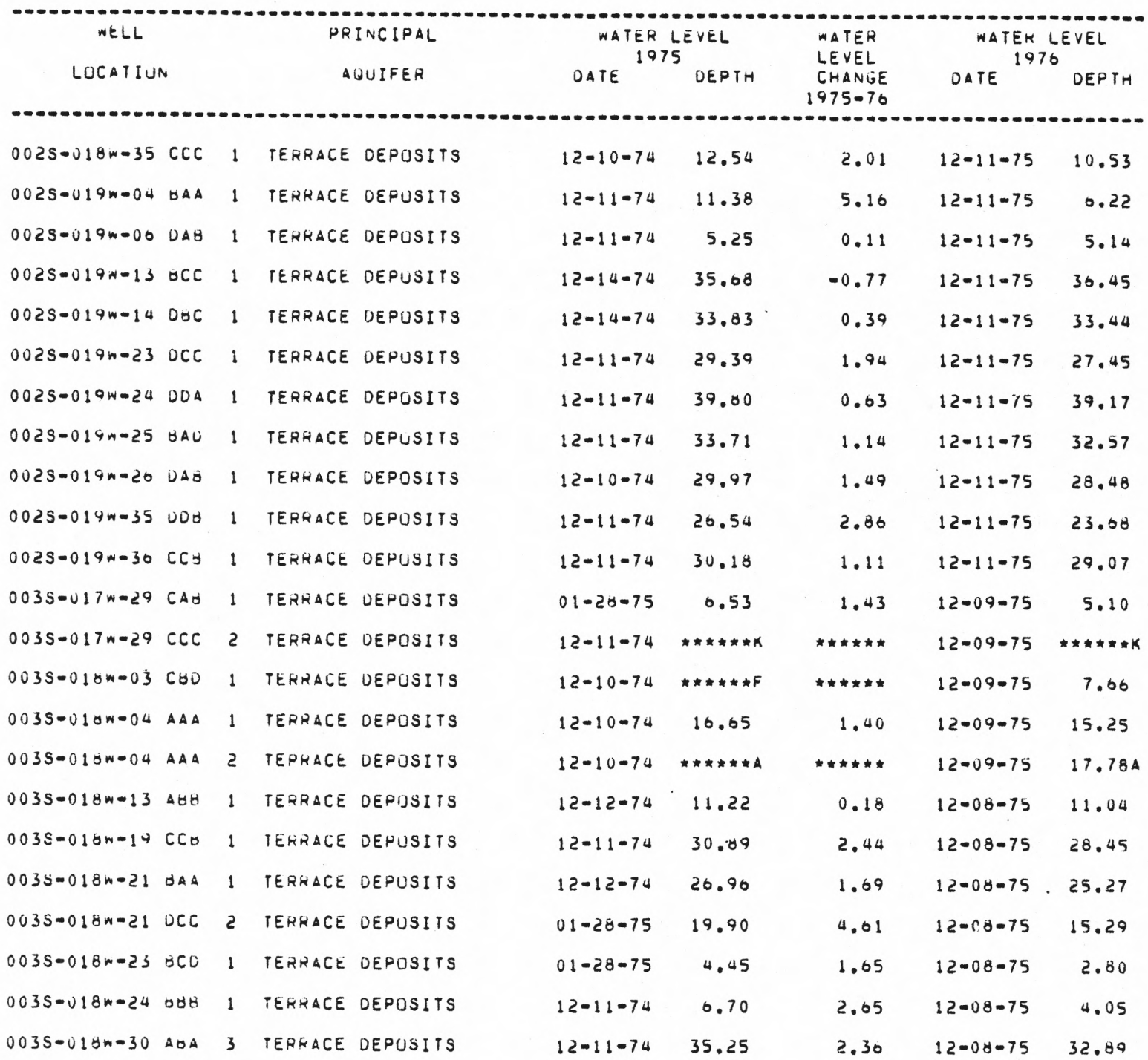


Table 1.--Water levels and water-level changes--Continued.

TILLMAN COUNTY

\begin{tabular}{|c|c|c|c|c|c|c|c|c|c|c|}
\hline \multirow{2}{*}{\multicolumn{2}{|c|}{ LOCATION }} & \multirow{2}{*}{\multicolumn{3}{|c|}{$\begin{array}{l}\text { PRINCIPAL } \\
\text { AQUIFEK }\end{array}$}} & & \multicolumn{2}{|c|}{$\begin{array}{l}\text { WATER LEVEL } \\
1975\end{array}$} & \multirow{2}{*}{$\begin{array}{l}\text { MATER } \\
\text { LEVEL } \\
\text { CHANGE } \\
1975=76\end{array}$} & \multicolumn{2}{|c|}{$\begin{array}{l}\text { WATEK LEVEL } \\
1975\end{array}$} \\
\hline & & & & & & DATE & DEPTH & & DATE & DEPTH \\
\hline $0035-018 n-30$ & CAA & 2 & TERRACE & DEPUSITS & & $12-11-74$ & 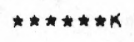 & $\star \star \star \star \star \star \star \star$ & $12-08-75$ & 29.49 \\
\hline $03 s-010 m-32$ & CDC & 1 & TERRACE & DEPOSITS & & $12-11-74$ & 32.84 & 3.01 & $12=08-75$ & 29.83 \\
\hline $035-018 w-32$ & DISA & 2 & TERRACE & DEPUSITS & & $12-11-74$ & 32.49 & 4.40 & $12-08-75$ & 28.03 \\
\hline $003 s=018 w-33$ & $\mathrm{CCB}$ & 1 & TERRACE & UEPOSITS & & $12-11-74$ & 31.84 & 5.32 & $12-08-75$ & 26.52 \\
\hline $0035-019 m-01$ & $B C D$ & 1 & TERRACE & VEPOSITS & & $12-10-74$ & 33.51 & 1.28 & $12-09-75$ & 32.23 \\
\hline $035-019 w=01$ & $D C A$ & 2 & TERRACE & OEPOSITS & & $12-10-74$ & 35.24 & 2.92 & $12-09-75$ & 32.32 \\
\hline $0035=019 m-02$ & $A 8 B$ & 1 & TERRACE & DEPOSITS & & $12-10-74$ & 29.18 & 1.89 & $12-09-75$ & 27.29 \\
\hline $0035-019 w=03$ & $\triangle A C$ & 2 & TERRACE & DEPOSITS & & $12-10-74$ & 16.23 & 4.66 & $12-09-75$ & 11.57 \\
\hline $0035-019 w-11$ & $D C A$ & 1 & TERRACE & DEPUSITS & & $12-10-74$ & 21.10 & 2.84 & $12=09-75$ & 18.26 \\
\hline $0035=019 m-13$ & $\forall C C$ & 1 & TERRACE & DEPDSITS & & $12-10-74$ & $\| * t$ & $\star \star \star \star \star \star \star \star$ & $12-08-75$ & $* * k$ \\
\hline $0035-019 w-14$ & $A A B$ & 1 & TERRACE & DEPOSITS & & $12-10-74$ & 21.66 & 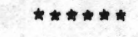 & $12-09-75$ & 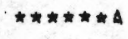 \\
\hline $003 s-019 w-22$ & $A A A$ & 1 & TERRACE & OEPOSITS & & & 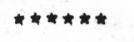 & $\star \star \star \star \star \star \star \star *$ & $12-09-75$ & 27.94 \\
\hline $0035-019 w-28$ & $A E B$ & 2 & TERRACE & DEPOSITS & & & $\star \star \star$ & $\hbar \star \star \star \star \star \star *$ & $12-09-75$ & 9.74 \\
\hline $003 s-0.19 w-36$ & $B A C$ & 1 & TERRACE & OEPDSITS & & $12-11-74$ & 22.30 & $\star \star \star \star \star \star * \star *$ & $12-08-75$ & $\star \star \star \star \star \star \star \star L L$ \\
\hline $0045=018 m=04$ & $A O A$ & 1 & IERRACE & DEPOSITS & & & $*$ & * & $12-08-75$ & $t^{\prime \prime}$ \\
\hline $0045-018 w=05$ & $A C U$ & $b$ & TERRACE & DEPUSITS & & $12-11-74$ & 33.94 & 4.59 & $12-18-75$ & 29.35 \\
\hline $0045-018 n-06$ & $\triangle O C$ & 1 & TERRACE & DEPOSITS & & $12-11-74$ & $\star \star \star \star \star \star \star \star k$ & $\star \star \star \star \star \star \star \star *$ & $12-08-75$ & $\# \star \star \star \star \star \star k K$ \\
\hline $0045-018 *-10$ & $A C A$ & 1 & TERRACE & DEPOSITS & & $12-11-74$ & $\star \star$ & $\Rightarrow x$ & $12=08-75$ & $x \times$ \\
\hline $0045-018 k=11$ & $A A C$ & 1 & TERRACE & DEPOSITS & & $12-11-74$ & 30.47 & 0.72 & $12-09-75$ & 29.75 \\
\hline $0045-019 w-01$ & $\Delta \Delta D$ & 1 & TERRACE & OEPOSITS & & $12-11-74$ & 32.86 & 3.75 & $12-08-75$ & 29.11 \\
\hline $001 N=017 w=00$ & DBA & 1 & TERRACE & OEPOSITS & & $01-09-75$ & 13.24 & 0.71 & $12-12-75$ & 12.53 \\
\hline $001 N-017 m=30$ & DCC & 1 & TERRACE & DEPUSITS & & $12-10-74$ & 17.10 & 2.00 & $12-11-75$ & 15.10 \\
\hline $01 N-017 m-31$ & DCU & 1 & TERRACE & DEPOSITS & & $12-10-74$ & 30.20 & 0.48 & $12-11-75$ & 29.72 \\
\hline
\end{tabular}


Table 1.-Water levels and water-level changes--Continued.

\begin{tabular}{|c|c|c|c|c|c|c|c|c|c|}
\hline \multirow{2}{*}{$\begin{array}{l}\text { WELL } \\
\text { LOCATIUN }\end{array}$} & & & \multirow{2}{*}{\multicolumn{2}{|c|}{$\begin{array}{l}\text { PKINCIPAL } \\
\text { AQUIFER }\end{array}$}} & \multicolumn{2}{|c|}{$\begin{array}{l}\text { WATER LEVEL } \\
1975\end{array}$} & \multirow{2}{*}{$\begin{array}{l}\text { WATER } \\
\text { LEVEL } \\
\text { CHANGE } \\
1975-76\end{array}$} & \multicolumn{2}{|c|}{$\begin{array}{l}\text { WATEK LEVEL } \\
1976\end{array}$} \\
\hline & & & & & DATE & DEPTH & & DATE & UEPTH \\
\hline $001 N=019 w-24$ & $\triangle A O$ & 1 & TERRACE & DEPUSITS & $01-09-75$ & 27.76 & 0.88 & $12-12-75$ & 26.88 \\
\hline $001 N=019 n-25$ & 880 & 1 & TERRACE & DEPOSITS & $12-09-74$ & 27.45 & 1.58 & $12-11-75$ & 25.87 \\
\hline $001 \mathrm{~N}=019 w=25$ & $\triangle C C$ & 2 & TERRACE & DEPUSITS & $12-09-74$ & 29.39 & 2.78 & $12-11-75$ & 26.61 \\
\hline $001 N=019 m-26$ & D8B & 3 & TERRACE & OEPOSITS & $12-09-74$ & 24.73 & 2.22 & $12-11-75$ & 22.51 \\
\hline $001 N-019 w-35$ & DoC & 1 & TERRACE & DEPUSITS & $12-09-74$ & 33.80 & 4.82 & $12-11-75$ & 28.98 \\
\hline $001 N-019 w-36$ & 808 & 1 & TERRACE & DEPOSITS & $12-09-74$ & 25.98 & 1.07 & $12-11-75$ & 24.91 \\
\hline $001 N=019 w=30$ & $\mathrm{CCB}$ & 2 & TERRACE & DEPOSITS & $12-09-74$ & $\star \star \star \star \star \star \star \star k$ & 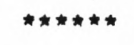 & $12-11-75$ & $\# \# \# \star \star \star \star M$ \\
\hline $002 N-017 W=19$ & DOA & 1 & TERRACE & UEPOSITS & $12-10-74$ & 26.98 & 1.41 & $12-12-75$ & 25.57 \\
\hline $002 N=017 w-20$ & $\partial C D$ & 1 & TERRACE & DEPOSITS & $01-09-75$ & 22.50 & 0.62 & $12-12-75$ & 21.88 \\
\hline $002 N-017 m=31$ & $A C A$ & 1 & TERRACE & DEPOSITS & & 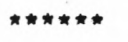 & 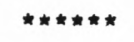 & $12-12-75$ & $\star \star \star \star \star \star \pitchfork \star M$ \\
\hline $002 N=017 n-31$ & $\cos$ & 1 & TERRACE & DEPOSITS & & 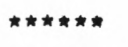 & 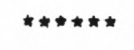 & $12-12-75$ & 17.45 \\
\hline $002 N=018 n=24$ & DO\& & 1 & TERRACE & DEPOSITS & $01-09-75$ & 20.99 & 0.42 & $12-12-75$ & 20.57 \\
\hline $002 N-018 w=36$ & $\forall 00$ & 1 & TERRACE & DEPOSITS & $12-10-74$ & 15.78 & 1.44 & $12-12-75$ & 14.34 \\
\hline
\end{tabular}


Table 1.-Water levels and water-level changes--Continued.

TILLMAN COUNTY

\begin{tabular}{|c|c|c|c|c|c|c|c|c|c|}
\hline \multirow{2}{*}{$\begin{array}{l}\text { NELL } \\
\text { LUCATIUN }\end{array}$} & \multirow{2}{*}{  } & \multirow{2}{*}{\multicolumn{3}{|c|}{$\begin{array}{l}\text { PRINCIPAL } \\
\text { AUUIFER }\end{array}$}} & \multicolumn{2}{|c|}{$\begin{array}{l}\text { WATER LEVEL } \\
1975\end{array}$} & \multirow{2}{*}{$\begin{array}{l}\text { NATER } \\
\text { LEVEL } \\
\text { CHANGE } \\
1975-76\end{array}$} & \multicolumn{2}{|c|}{$\begin{array}{l}\text { WATEK LEVEL } \\
1976\end{array}$} \\
\hline & & & & & DATE & DEPTH & & DATE & DEPTH \\
\hline $001 N=018 m=01$ & CBA & 1 & TERRACE & DEPOSITS & $12-10-74$ & 12.88 & 0.98 & $12-12-75$ & 11.90 \\
\hline $001 N=016 N=02$ & $C C A$ & 2 & TERRACE & UEPOSITS & $12-10-74$ & $\star \star \star \star \star \star \star \star \star K$ & 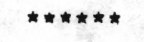 & $12-12-75$ & 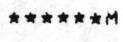 \\
\hline $001 N-018 w=03$ & $A \cup A$ & 1 & TERRACE & DEPOSITS & $12-10-74$ & 0.55 & -0.47 & $12-12-75$ & 9.02 \\
\hline $001 N-018 n-10$ & 000 & 1 & TERQACE & DEPOSITS & $12-10-74$ & 17.88 & 0.19 & $12-12-75$ & 17.69 \\
\hline $001 N=018 m-11$ & $\Delta \otimes \theta$ & 1 & TERRACE & OEPOSITS & $12-10-74$ & 12.30 & 0.16 & $12-12-75$ & 12.14 \\
\hline $001 N-018 w-11$ & $C C A$ & 1 & TERRACE & DEPUSITS & $01-09-75$ & 14.44 & -0.01 & $12-12-75$ & 14.45 \\
\hline $001 N=018 N=12$ & CAA & 1 & TERKACE & DEPOSITS & $01-09-75$ & $\star \star \star \star \star \star \star \star x$ & 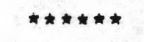 & $12-12-75$ & 9.90 \\
\hline $001 N=018 w-13$ & $\triangle B A$ & 1 & TERRACE & DEPOSITS & $12-10-74$ & 5.51 & 3.57 & $12-12-75$ & 1.94 \\
\hline $001 N-018 w-14$ & UAD & 3 & TERKACE & DEPOSITS & $12-10-74$ & 21.56 & 0.37 & $12-11-75$ & 21.19 \\
\hline $001 N=018 N-10$ & NOB & 1 & TERRACE & DEPOSITS & $12-09-74$ & 20.52 & 0.80 & $12-11-75$ & 19.72 \\
\hline $001 N=010 w-17$ & Cot & 1 & TERRACE & DEPUSITS & $12=09-74$ & 18.61 & 0.93 & $12-12-75$ & 17.68 \\
\hline $001 N-018 m-18$ & $A \triangle A$ & 1 & TERRACE & DEPOSITS & $12-09-74$ & 14.52 & 1.02 & $12-12-75$ & 13.50 \\
\hline $001 N-010 N-19$ & $C C A$ & 1 & TERRACE & DEPOSITS & $12-09-74$ & 25.82 & $\star \star \star \star \star \star \star \star$ & $12-11-75$ & $\star$ \\
\hline $001 N-018 w-20$ & $\csc$ & 1 & TERRACE & DEPOSITS & $12-09-74$ & 33.05 & 1.75 & $12-11=75$ & 31.30 \\
\hline $001 N=018 w-21$ & $A G \Delta$ & 1 & TERRACE & DEPISITS & $12-09-74$ & 10.23 & 1.70 & $12-11-75$ & 14.47 \\
\hline $001 N-018 w-22$ & 000 & 1 & TERRACE & DEPOSITS & $12-10-7 a$ & 15.35 & 1.34 & $12-11-75$ & 14.01 \\
\hline $001 N-018 w-54$ & $\operatorname{COC}$ & 1 & TERTRACE & DEPOSITS & $12-10-74$ & 4.54 & 0.54 & $12-12-75$ & 4.00 \\
\hline $001 N-018 w-28$ & DAA & 3 & TERRACE & DEPOSITS & $12-09-74$ & 10.83 & 2.15 & $12-11-75$ & 16.68 \\
\hline $001 N-018 N-29$ & odo & 1 & TERKACE & DEPOSITS & $12=09-74$ & 25.45 & 1.45 & $12-11-75$ & 24.00 \\
\hline $001 N=018 w-30$ & $\triangle C A$ & 1 & TERRACE & DEPOSITS & $12-09-74$ & 26.50 & 2.55 & $12-11-75$ & 23.95 \\
\hline $001 N-018 n-31$ & $00 \mathrm{C}$ & 1 & TERRACE & DEPOSITS & $12-09-74$ & 21.94 & 1.87 & $12-11=75$ & 20.07 \\
\hline $001 N=018 w-33$ & $O A C$ & 1 & TERRACE & OEPOSITS & $12-09-74$ & 17.29 & 1.54 & $12-11-75$ & 15.75 \\
\hline $001 N-018 w-35$ & cou & 4 & TERRACE & DEPOSITS & $12-10=74$ & 14.97 & 3.20 & $12-11-75$ & 11.77 \\
\hline
\end{tabular}


Table 1.--Water levels and water-level changes--Continued.

WASIIITA COUNTY

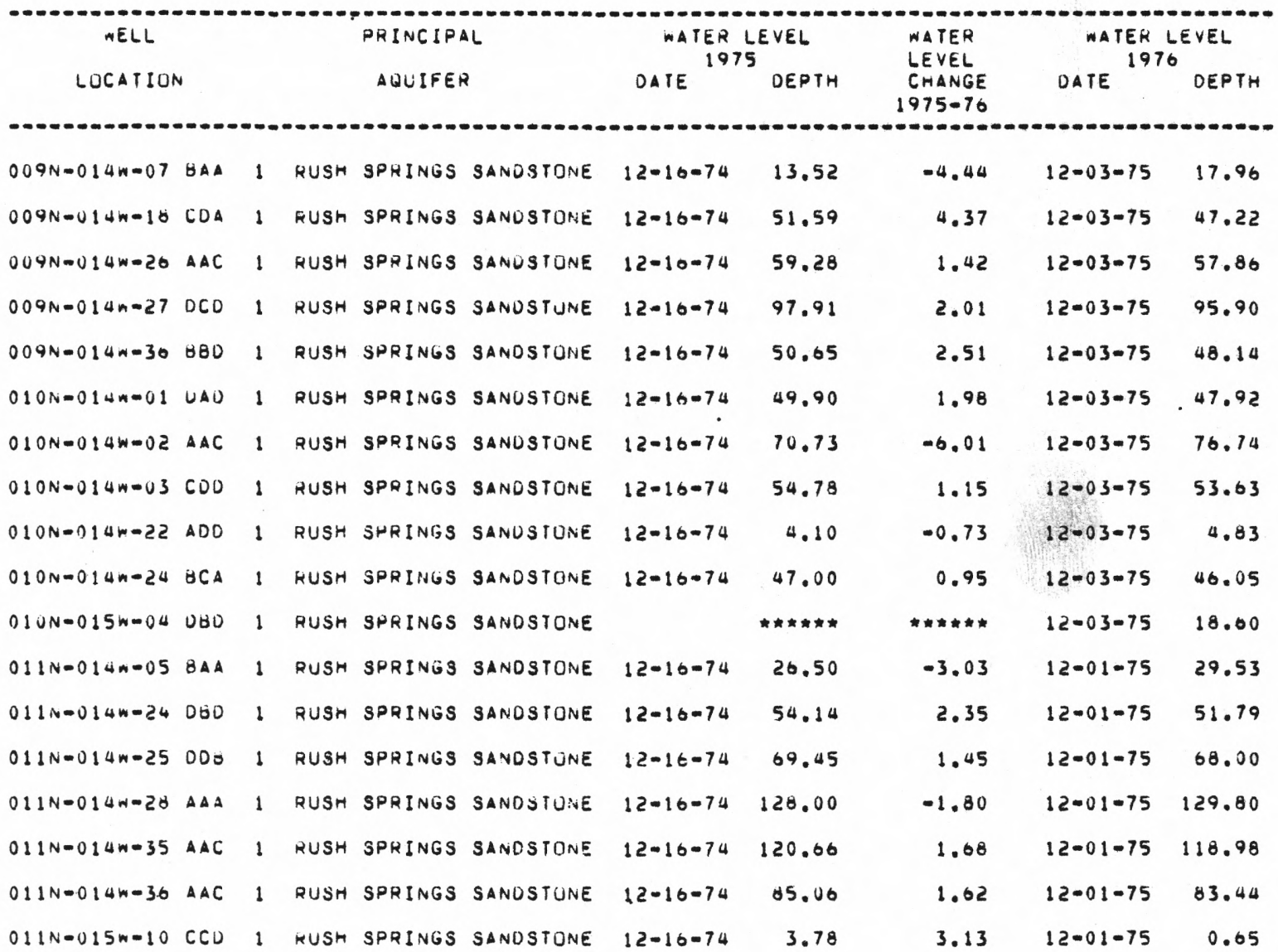

WUODS COUNTY

\begin{tabular}{|c|c|c|c|c|c|c|c|c|}
\hline \multirow{2}{*}{\multicolumn{2}{|c|}{$\begin{array}{c}\text { WELL } \\
\text { LOCATIUN }\end{array}$}} & \multirow{2}{*}{\multicolumn{2}{|c|}{$\begin{array}{l}\text { PRINCIPAL } \\
\text { AGUIFER }\end{array}$}} & \multicolumn{2}{|c|}{$\begin{array}{l}\text { WATER LEVEL } \\
1975\end{array}$} & \multirow{2}{*}{$\begin{array}{l}\text { WATEF } \\
\text { LEVEL } \\
\text { CHANGE } \\
1975-70\end{array}$} & \multicolumn{2}{|c|}{$\begin{array}{c}\text { WATER LEVEL } \\
1976\end{array}$} \\
\hline & & & & DATE & DEPTH & & DATE & DEPTH \\
\hline $023 N-013 N-17$ & Cot & 1 & TERRACE UEPOSITS & & 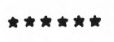 & 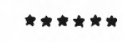 & $01-15-76$ & 0.33 \\
\hline $024 N-014 n-23$ & vou & 1 & TERKACE DEPUSITS & $01-23-75$ & 32.44 & 0.84 & $01-15-76$ & 31.60 \\
\hline $025 N-015 N=29$ & CAO & 1 & TERRACE DEPOSITS & & 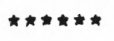 & $\star \star \star \star \star \star \star \star$ & $01-15-70$ & 39.59 \\
\hline $25 N=015 w=29$ & OOA & 1 & TERRACE UEPUSITS & $01-23-75$ & 21.05 & $\# \star \star \star \star \star \star$ & $01-15-76$ & 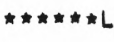 \\
\hline
\end{tabular}


Table 1.--Water levels and water-level changes--Continued.

\begin{tabular}{|c|c|c|c|c|c|c|c|c|c|}
\hline & & & PRI & INCIPAL & $\begin{array}{r}\text { WATER } \\
197\end{array}$ & EVEL & & $\begin{array}{r}\text { WATER } \\
197\end{array}$ & 6 \\
\hline LOCATION & & & & JUIFER & DATE & UEPTH & $\begin{array}{l}\text { CHANGE } \\
1975-76\end{array}$ & DATE & OEPTH \\
\hline $020 N-017 N=05$ & $C O C$ & 1 & TERKACE & LEPOSITS & $01-15-75$ & 5.28 & -0.55 & $12-11-75$ & 5.83 \\
\hline $020 N-019 W=01$ & BAA & 1 & TERRACE & DEPOSITS & & 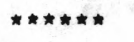 & 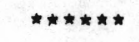 & $12-11-75$ & 198.46 \\
\hline $020 N-020 W=30$ & 886 & 1 & TERRACE & CEPOSITS & $01-15-75$ & $\star \star \star \star \star \star \star F F$ & $\hbar \star \star \star \star \star \star *$ & $12-11-75$ & 34.18 \\
\hline $020 N-022 W-30$ & $\Delta 00$ & 1 & PERRACE & DEPOSITS & $01-15-75$ & $\star \star \star \star \star \star F F$ & 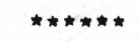 & $12-11-75$ & 97.75 \\
\hline $021 N=018 W=27$ & $A C B$ & 1 & TERAACE & LEPUSITS & $01-15-75$ & 39.32 & 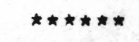 & $12-11=75$ & $\star \star \star \star \star \star \star \star A$ \\
\hline $021 ;-01+m-31$ & $03 A$ & 1 & TERIACEE & DEPUSIIS & $01-15-75$ & 5.02 & -0.92 & $12-11-75$ & 0.74 \\
\hline $021 v-017 x-12$ & $0 \times 0$ & 1 & TERRACE & UEPOSITS & $01-15-75$ & 0.01 & -1.25 & $12-11-75$ & 7.86 \\
\hline $021 N-021 N-12$ & $\cos$ & 1 & TERRACE & DEPUSITS & $01-15-75$ & 14.97 & -0.06 & $12-11-75$ & 15.03 \\
\hline $021 N-022 w-23$ & 300 & 1 & TERRACE & UEPUSITS & $01-15-75$ & 30.13 & 0.53 & $12-11-75$ & 29.00 \\
\hline $022 N=018 n-33$ & $\operatorname{ccc}$ & 1 & TERRACE & OEPOSITS & $01-15-75$ & 27.07 & 1.94 & $12-11-75$ & 25.73 \\
\hline $022 N=020 N=25$ & $B O A$ & 1 & TERRACE & JEPOSITS & $01-15-75$ & 3.39 & -0.76 & $12-11-75$ & 4.15 \\
\hline $022 N=022 N-14$ & $\operatorname{ccc}$ & 1 & TERHACE & DEPUSITS & $01-15-75$ & 20.26 & 0.35 & $12-11-75$ & 27.91 \\
\hline $023 N-1) 19 n=22$ & $A A C$ & 1 & TERRACE & OEPOSITS & $01-15-75$ & 38.72 & -0.99 & $12-11-75$ & 39.71 \\
\hline $023 n-019 m-33$ & DOd & 1 & TERRACE & DEPOSITS & $01-15-75$ & $\star \star \star \star \star \star \star \star L L$ & 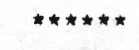 & & 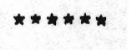 \\
\hline $023 N-020 n=05$ & OBA & 1 & TERKACE & VEPUSITS & $01-15-75$ & $\star \star \star \star \star \star \star \star \star \Delta$ & 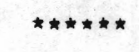 & $12-10-75$ & 32.29 \\
\hline $323=020=19$ & Coj & 1 & TERRACE & LEPOSITS & $v 1-15-75$ & 4.09 & 0.10 & $12-10-7 s$ & 3.93 \\
\hline $124-12 u n-14$ & uns & 1 & TERMALE & DEFUSIIS & $01-15-75$ & 50.30 & $\star \star \star \star * \star \star \star *$ & $12-10-73$ & $* *$ L \\
\hline $02+10-422 n=10$ & $\operatorname{ccc}$ & 1 & TEARACE & DEPUSITS & $01-14=75$ & 12.73 & 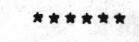 & $12-10-75$ &  \\
\hline
\end{tabular}


